Mon. Not. R. Astron. Soc. 000, 000-000 (0000) Printed 10 September $2018 \quad$ (MN LATEX style file v2.2)

\title{
Estimating gas masses and dust-to-gas ratios from optical spectroscopy
}

\author{
Jarle Brinchmann ${ }^{1,2 \star}$, Stéphane Charlot $^{3}$, Guinevere Kauffmann ${ }^{4}$, \\ Tim Heckman ${ }^{5}$, Simon D. M. White ${ }^{4}$, Christy Tremonti ${ }^{6}$ \\ ${ }^{1}$ Leiden Observatory, Leiden University, P.O. Box 9513, 2300 RA, Leiden, The Netherlands \\ ${ }^{2}$ Centro de Astrofisica, Universidade do Porto, Rua das Estrelas, 4150-762 Porto, Portugal \\ ${ }^{3}$ UPMC-CNRS, UMR7095, Institut d'Astrophysique de Paris, F-75014, Paris, France \\ ${ }^{4}$ Max-Planck Institut für Astrophysik, 85741 Garching, Germany \\ 5 Johns Hopkins University, Baltimore, Maryland 21218, USA \\ 6 Department of Astronomy, University of Wisconsin-Madison, 1150 University Ave, Madison, WI 53706, USA
}

10 September 2018

\begin{abstract}
We present a method to estimate the total gas column density, dust-to-gas and dust-tometal ratios of distant galaxies from rest-frame optical spectra. The technique exploits the sensitivity of certain optical lines to changes in depletion of metals onto dust grains and uses photo-ionization models to constrain these physical ratios along with the metallicity and dust column density. We compare our gas column density estimates with $\mathrm{HI}$ and $\mathrm{CO}$ gas mass estimates in nearby galaxies to show that we recover their total gas mass surface density to within a factor of 2 up to a total surface gas mass density of $\sim 75 \mathrm{M}_{\odot} \mathrm{pc}^{-2}$. Our technique is independent of the conversion factor of $\mathrm{CO}$ to $\mathrm{H}_{2}$ and we show that a metallicity dependent $X_{\mathrm{CO}}$ is required to achieve good agreement between our measurements and that provided by $\mathrm{CO}$ and $\mathrm{H}$. However we also show that our method can not be reliably aperture corrected to total gas mass. We calculate dust-to-gas ratios for all star-forming galaxies in the Sloan Digital Sky Survey Data Release 7 and show the resulting dependence on metallicity agrees well with the trend inferred from modelling of the dust emission of nearby galaxies using far-IR data. We also present estimates of the variation of the dust-to-metal ratio with metallicity and show that this is poorly constrained at metallicities below 50 per cent solar. We conclude with a study of the inventory of gas in the central regions, defined both in terms of a fixed physical radius and as a fixed fraction of the half-light radius, of $\sim 70,000$ star-forming galaxies from the Sloan Digital Sky Survey. We show that their central gas content and gas depletion times are not accurately predicted by a single parameter, but in agreement with recent studies we find that a combination of the stellar mass and some measure of central concentration provides a good predictor of gas content in galaxies. We also identify a population of galaxies with low surface densities of stars and very long gas depletion times.
\end{abstract}

Key words: galaxies - galaxies: evolution - galaxies: individual - galaxies: fundamental parameters - galaxies: ISM

\section{INTRODUCTION}

The baryonic content of a galaxy exists in a range of states, from cold molecular gas to diffuse hot gaseous haloes, yet for most galaxies our knowledge of the baryon content is biased towards the stars. Indeed, the last decade

^ jarle@strw.leidenuniv.nl has seen a massive increase in our understanding of the stellar content of galaxies over a wide range in redshift (e.g. Brinchmann \& Ellis 2000; Dickinson et al. 2003; Baldry et al. 2008; Marchesini et al. 2009; Shaplev 2011, for a review). Stellar masses and star formation rates are regularly obtained from broad-band photometry or optical spectroscopy for large samples of galaxies (e.g. Bell \& de Jong 2001; Kauffmann et al. 2003b; Brinchmann et al. 2004; 
Salim et al. 2007; Blanton \& Roweis 2007; Pforr et al. 2012; Maraston et al. 2012), but to fully understand the baryon cycle in galaxies we need to also understand the distribution of cold gas in galaxies.

The atomic gas content of galaxies can be traced using the $21 \mathrm{~cm}$ line of $\mathrm{HI}$, and integrated $\mathrm{HI}$ masses are now available for large samples of galaxies from $\mathrm{H} \mathrm{I}$-selected surveys such as HIPASS (Barnes et al. 2001) and ALFALFA (Havnes et al. 2011) and targeted surveys such as the GASS survey (Catinella et al. 2012), which has a uniform gas fraction limit rather than a gas mass limit. Resolved H I maps are available for smaller, but still substantial samples of galaxies such as WHISP (van der Hulst et al. 2001), the Ursa Major Cluster survey (Tully et al. 1996; Verheiien \& Sancisi 2001) and the THINGS survey (Walter et al. 2008).

The cold molecular gas is mostly composed of $\mathrm{H}_{2}$, but given its lack of transitions that can be excited at low temperatures, molecular gas measurements are mostly done using $\mathrm{CO}$ as a proxy for $\mathrm{H}_{2}$. Single-dish $\mathrm{CO}$ surveys have generally been targeted (e.g. Braine et al 1993; Boselli 1997) but for somewhat inhomogeneous samples. A notable exception here is the COLDGASS survey (Saintonge et al. 2011a) which obtained CO observations for galaxies from the GASS survey. As with H I, resolved CO studies have a long history (e.g. Young \& Scoville 1991; Young et al. 1995), recent examples are The Berkeley Illinois Maryland Association Survey of Nearby Galaxies (BIMA SONG; Helfer et al. 2003) interferometric maps of 44 spiral galaxies, the Nobeyama survey of 40 spiral galaxies Kuno et al. (2007) and the HERA CO Line Extragalactic Survey (HERACLES) by Leroy et al. (2009b) which mapped 18 nearby galaxies with extensive multi-wavelength data.

These methods are powerful but they have important drawbacks: While H I traces the dominant ingredient in the atomic gas, it is very challenging to detect significant numbers of individual galaxies in H I past $z \sim 0.3$ with current facilities (e.g. Jaffé et al. 2012; Verheijen et al. 2010; Catinella et al. 2008). In contrast, CO observations can be done out to significant redshifts, but they do not trace the dominant component of the molecular gas and a conversion factor between the column density of $\mathrm{CO}$ and $\mathrm{H}_{2}\left(X_{\mathrm{CO}}\right)$ or CO luminosity and total molecular mass $\left(\alpha_{\mathrm{CO}}\right)$ must be used. Both $\alpha_{\mathrm{CO}}$ and $X_{\mathrm{CO}}$ are believed to vary considerably with metallicity and ionisation conditions (e.g. Israel 1997b; Boselli et al. 2002; Israel 2005; Lerov et al. 2009a), although a quantitative understanding of this effect is only now becoming available (e.g. Leroy et al. 2011; Genzel et al. 2012).

In part due to these constraints, and also because $\mathrm{HI}$ and CO surveys are very time-consuming, alternative methods have been proposed to infer gas content in galaxies. These can be roughly divided into methods that make use of scaling relations to infer gas content, and those that measure gas content using a more distant tracer than CO. The former class has a long history and sprang out of the realisation that there is a good correlation between galaxy colour and the ratio of H I mass to B-band luminosity (Roberts 1969; Bothun 1984), or alternatively between galaxy morphology and $M_{\mathrm{H}} / L_{B}$ (Roberts \& Haynes 1994). This technique was used by Bell et al. (2003) to infer a baryonic mass function for the low redshift, $z<0.2$, Universe. Similarly Kannappan (2004, K04) and Zhang et al. (2009, Z09) have provided relations between colour and $\mathrm{HI}$ gas-fractions which can be applied to large samples of galaxies. This has the advantage of being easy to apply to large samples of objects (e.g. Kauffmann et al. 2010; Zhang et al. 2012). A similar technique in this class is to invert the empirically found relationship between star formation rate and gas surface density, the Kennicutt-Schmidt relation Kennicutt 1998, and references therein). This requires an estimate of the star formation rate which is then used to infer a gas mass (e.g. Erb et al. 2006; Tremonti et al. 2004; Puech et al. 2010). These relations do however rely on local calibration samples and are therefore of questionable use for applications at higher redshift. Even more importantly they are calibrated to give average trends and are not suitable for detecting outliers or peculiar systems. We will discuss these issues further in section 5.5.

The second class of methods aims instead to infer gas content via a tracer of gas content. The most widely used technique in this class is to make use of dust emission to infer gas content, building on the observation by Boulanger et al. (1985) that a comparison of IRAS and H I maps implied that dust is a reliable tracer of cold gas. It has been used to estimate gas content in the Milky Way (e.g. Dame et al. 2001), and in nearby galaxies by a number of authors (e.g. Israel 1997a b, 2005; Lerov et al. 2009a; Boquien et al. 2013) and it has been extended to sub-mm observations (James et al. 2002) and more recently to Herschel data (e.g. Eales et al. 2010, 2012, see also Roman-Duval et al 2010). These models depend on dust properties, such as the emissivity and temperature, and on the typical beam sizes for sub-mm and far-IR observations which are considerably larger than the resolution obtained in the optical. Modelling the dust emission can, however, yield a plethora of additional information (e.g. Draine et al. 2007; da Cunha et al. 2008). Of particular interest to this paper is the ability to constrain the dustto-gas ratio of the interstellar medium (e.g. Aniano et al. 2012).

Here we will discuss a related technique, but instead of exploiting the dust emission, we will make use of the dust absorption. As we will discuss below this can be applied to large samples of galaxies, is relatively insensitive to the detailed properties of the dust grains, and like the dust emission measure, is sensitive to the total gas content and dust-to-gas ratio. At low redshift it therefore offers a promising complement to direct detection of gas for characterising its properties, while at higher redshift it offers a simple method to measure the gas content and dust-togas ratios of large samples of galaxies. As we will outline below the method, being based on optical data, generally has higher spatial resolution than interferometric $\mathrm{HI}$ and CO maps, but it is naturally limited to the stellar disk of galaxies since it is an absorption technique. Thus most of our results in the final part of the paper will focus on the gas content in the central few kpc of galaxies, nicely complementing the integrated measurements gas measurements provided by single-dish observations.

The plan of the paper is as follows: In sections 2 and 3 below we will discuss our method for modelling gas content in some detail, including the importance of priors on our model parameters. This will result in a model which links dust content, metallicity and dust-to-metal ratio to produce an estimate of gas column density in a given system. We review the data we will use to validate our technique in section 4. and emphasise the importance of the chosen at- 
tenuation curve. Section 5 is devoted to tests of our method and we start with a study of how the dust-to-metal and dust-to-gas ratios in galaxies depend on redshift, showing good agreement with modelling of dust emission in nearby galaxies. We then show that our gas column densities agree with those inferred for the same regions from $\mathrm{H}$ I+CO maps in nearby galaxies and exploit the aperture trends inherent in Sloan Digital Sky Survey (SDSS) spectroscopic data to check that we can recover well-known scaling relations. In section 6 we discuss the gas content and gas depletion times in the central regions of $\sim 70,000$ galaxies in the local Universe, selected from all $\sim 200,000$ star-forming galaxies in the DR7. We conclude in section 7 . Throughout we adopt a cosmology with $H_{0}=70 \mathrm{~km} \mathrm{~s}^{-1} \mathrm{Mpc}^{-1}, \Omega_{m}=0.3$ and $\Omega_{\Lambda}=0.7$. Where relevant we adopt a Kroupa initial mass function (Kroupa 2001). We will denote surface densities by $\Sigma$.

\section{ESTIMATING GAS MASSES FROM DUST ATTENUATION}

From observations it is clear that dust and gas trace each other fairly closely, at least when averaged over sufficiently large regions. Here we wish to build on this to construct a probe of gas content using the dust in absorption as a probe of the gas column density and exploiting the temperature dependence of emission lines to place constraints on the dustto-gas ratio; together they will then provide an estimate of the effective gas column density in the region probed by the spectrum. We note that our focus here will be on the attenuation of emission lines only, but if a separate constraint on the absorption of the stellar continuum can be had (e.g. Kauffmann et al. 2003b), further constraints on the model can in principle be obtained.

We adopt the simple dust attenuation model by Charlot \& Fall (2000, CF00) and it is therefore convenient to distinguish between attenuation in the stellar "birth clouds" (i.e. molecular clouds), $\tau_{\mathrm{BC}}$ and in the "ambient" (i.e. diffuse) ISM, $\tau^{\text {ISM }}$.

$\tau_{V}=\tau_{V}^{B C}+\tau_{V}^{\mathrm{ISM}}$

CF00 found a good fit to their sample of galaxies with the assumption that the attenuation in the diffuse ISM is typically only about $1 / 3$ of the total attenuation affecting young stars (see also da Cunha et al. 2008), but the actual value turns out not to matter for our argument here (but see the discussion in section 3.2).

We now follow Charlot \& Longhetti 2001, CL01) and introduce a new variable, $\xi$, which is the dust-to-metal ratio of the ionised gas,

$\xi=\frac{M_{d}}{M_{Z}}$,

where $M_{d}$ is the dust mass and $M_{Z}$ is the mass in metals. Alternatively this can be written as

$\xi=\frac{\sum_{i} m_{i} \delta\left(\mathrm{X}_{i}\right)\left(\frac{\mathrm{X}_{i}}{\mathrm{H}}\right)_{c}}{\sum_{i} m_{i}\left(\frac{\mathrm{X}_{i}}{H}\right)_{c}}$,

where $\delta\left(\mathrm{X}_{i}\right)$ is the linear depletion of element $\mathrm{X}_{i}$, and $m_{i}$ is the mass of element $\mathrm{X}_{i}$ and where $\left(\mathrm{X}_{i} / \mathrm{H}\right)_{c}$ is the cosmic, ie. undepleted, abundance of element $\mathrm{X}_{i}$. We will return to the question of expected values for $\xi$ below. Note that the combination $\xi Z$, with $Z$ being the total metallicity, provides a measure of the dust-to-gas ratio, and we will return to this in section 5.2

The surface mass density of gas contributed by the birth clouds (assumed spherical) can thereby be derived using the definition of $\xi=\Sigma_{d} / \Sigma_{Z}$ where $\Sigma_{d}$ is the surface density of dust and $\Sigma_{Z}$ that of metals, and we have made the assumption that the effective scale-length of metals and dust is the same. In addition the metallicity can be written $Z=\Sigma_{Z} / \Sigma_{\text {gas }}$, which taken together gives

$\Sigma_{\text {gas }}^{B C}=\tau_{V}^{B C} m_{d} /\left(\sigma_{d} \xi Z\right)$,

where $m_{d}$ is the mass of the dust grain and $\sigma_{d}$ its optical cross-section - these should be viewed as effective, angleaveraged, quantities in the sense adopted by CF00.

According to $\mathrm{CF} 00$, the model that produced the better fit to their attenuation curve was on based on a Poisson distribution of discrete clouds. Let $\tau_{V}^{c}$ be the optical depth per cloud and $\bar{n}$ be the mean number of clouds encountered along different lines of sight. The observations of starburst galaxies favour $0.1<\tau_{V}^{c}<0.5$ with a best-fit value of 0.3 . With these additional assumptions about the optical properties and the spatial distribution of dust, we can relate $\tau_{V}^{\text {ISM }}$ to $\Sigma_{\text {gas }}^{\text {ISM }}$. For a Poisson distribution, the effective absorption optical depth in the diffuse ISM is (CF00; Eq 25) simply

$\tau_{V}^{\mathrm{ISM}}=\bar{n}\left[1-\exp \left(-\tau_{V}^{c}\right)\right]$,

with typically $\tau_{V}^{c}=0.3$. Now, to go from a Poisson distribution for the probability density of absorption, to a Poisson distribution of gas clouds, we require a new assumption: that scattering is mostly forward. This seems to be well supported by both models and observations of interstellar dust in the Milky Way. Draine (2003) compared predictions of $\langle\cos \theta\rangle$ from models with a range of observations and found that $\langle\cos \theta\rangle>0.6$ in all environments at the optical and UV wavelengths of interest to us (see also Gordon (2004) and references therein). In this case, the mean surface mass density of gas along different lines of sight in the diffuse ISM is simply:

$\Sigma_{\text {gas }}^{\mathrm{ISM}}=\bar{n} \Sigma_{\text {gas }}^{c}$

where $\Sigma_{\text {gas }}^{c}$ is the surface mass density of individual clouds (note that, strictly, we have adopted here a Poisson distribution of identical, face-on screens rather than one of spherical clouds). Substituting $\bar{n}$ from equation (5), we then have

$\Sigma_{\text {gas }}^{\mathrm{ISM}}=\frac{\tau_{V}^{\mathrm{ISM}} \Sigma_{\text {gas }}^{c}}{1-\exp \left(-\tau_{V}^{c}\right)}$.

The mass density of an individual cloud can be inferred as in equation (4) above as

$\Sigma_{\text {gas }}^{c}=\tau_{V}^{c} m_{d} /\left(\sigma_{d} \xi Z\right)$

We can then substitute this in equation (7) and expand in powers of $\tau_{V}^{c}$. For optically thin clouds (recall $\tau_{V}^{c}=0.3$ ), we can write

$\Sigma_{\text {gas }}^{\mathrm{ISM}} \approx \tau_{V}^{\mathrm{ISM}} m_{d} /\left(\sigma_{d} \xi Z\right)$.

It is important to note that this is only an approximate formula, which breaks down for more optically thick clouds, but for $\tau_{V}^{c}=0.33$, the next term is only 17 per cent of the 
equation above. With all the assumptions listed above, we can then write

$$
\begin{aligned}
\Sigma_{\text {gas }} & =\Sigma_{\text {gas }}^{B C}+\Sigma_{\text {gas }}^{\mathrm{ISM}} \\
& =\frac{\left(\tau_{V}^{B C}+\tau_{V}^{\mathrm{ISM}}\right) m_{d}}{\sigma_{d} \xi Z} \\
& =\frac{\tau_{V} m_{d}}{\sigma_{d} \xi Z}
\end{aligned}
$$

For $m_{d}$ and $\sigma_{d}$ corresponding to Galactic-type dust, we adopt a mass-weighted mean grain radius of $a=0.1 \mu \mathrm{m}$ (using a MRN distribution between 0.005 and 0.25 micron), $\sigma_{d} \approx \pi a^{2}$ (since we assumed forward scattering) and $\rho_{d} \approx 3 \mathrm{~g} \mathrm{~cm}^{-3}$ (the mean mass density of silicates and graphite grains). This yields

$\Sigma_{\text {gas }} \approx 0.2 \frac{\tau_{V}}{\xi Z} \mathrm{M}_{\odot} \mathrm{pc}^{-2}$

The numerical pre-factor in equation (13) is fairly robustly determined as it only depends on the mass-weighted radius to the first power as well as the reasonably welldetermined density of carbonaceous and silicate grains. Using the Weingartner \& Draine (2001, WD01) model we find that for $R_{V}=3.1$ the pre-factor ranges from 0.17 to 0.23 across the various $\mathrm{C}$ abundances considered by those authors. For LMC and SMC dust from the WD01 modelling, the pre-factor ranges between 0.21 and 0.26 . Thus we conclude that the overall scaling is robustly determined, at least across a range of metallicities from the Milky-Way to the SMC. We will adopt equation (13) in the following. In passing we comment that if we have a separate constraint on $\Sigma_{\text {gas }}^{\text {ISM }}$ from e.g. the attenuation of the stellar continuum, it might be possible to extend this method to provide an estimate of the relative gas content in the ISM and in birth clouds and it would also allow constraints to be placed on the gas column densities in galaxies without significant ongoing star formation.

Despite the overall robustness of the scaling factor to the dust grain properties, the derivation above does depend on a number of uncertain assumptions so it is essential to test the results carefully. In particular it is not clear at what density the simplification made in equation (9) will break down, leading to an underestimate of the gas density. We are also sensitive to the exact extinction curve being adopted as well as the metallicity estimator used and we will discuss this further in section 5 Finally, at very low column densities our modelling will break down at some point. Since the technique is based on absorption it might be expected to be more sensitive than emission-based probes such as $\mathrm{CO}$ or $\mathrm{H}$, but we know less about the properties of the attenuation curve at low gas densities thus our systematic uncertainties could become important here and the method as discussed here relies on a background source with emission lines which might not be present at very low densities.

It is worth emphasising that this approach is sensitive to the total, ie. atomic plus molecular, gas surface mass density in the observed region. This is crucial for testing because it implies that using only $\mathrm{H}$ i is insufficient where significant contributions to the gas mass are expected to be in molecular form.

\section{DERIVATION OF $\Sigma_{\text {gas }}$ THROUGH FITTING TO EMISSION LINES}

We now discuss briefly how we use equation (13) to constrain $\Sigma_{\text {gas }}$. We need a framework to constrain the metallicity, the dust attenuation and the dust-to-metal ratio of the ionised gas. Furthermore we have to take into account the fact that these quantities likely will be correlated. To address these issues we follow closely the approach outlined in Brinchmann et al. (2004, hereafter B04) and use a grid of the models derived by Charlot \& Longhetti (2001). We will also discuss the choice of priors and a number of uncertain ingredients in our modeling and comparisons. For ease of reference this is summarised in Table 2 below.

The CL01 models provide predictions for emission line strengths, combining evolving galaxy spectra from the Bruzual \& Charlot (1993) models with the Cloudy photoionisation code (Ferland et al. 1998). The models are parametrised by five main parameters and are calculated on a regular grid in all these parameters (see table 2 in B04). For our work here we need four of these parameters: the ionisation parameter, $U$, the dust-to-metal ratio in the ionised gas, $\xi$, the dust attenuation in the $V$ band, $\tau_{V}$, and the metallicity, $Z$, note that this is the total metallicity including metals depleted onto dust grains and therefore differs from the gas-phase metallicity. We then fit our grid of CL01 models to optical emission lines; when available we use the main optical strong lines [O II], H $\beta$, [O III]4959,5007, $\mathrm{H} \alpha,[\mathrm{N} \mathrm{II}] 6584$ and [S II] 6717,6731 , we will discuss their importance later. Note that with the abundance patterns used in the CL01 models, the oxygen abundance of the sun is $12+\log (\mathrm{O} / \mathrm{H})_{\odot}=8.82$.

The $\xi$ parameter, the dust-to-metal ratio of the ionised gas, is not commonly used in photo-ionisation calculations and warrants a more extensive discussion. It is a linear depletion parameter and its average value, which is what we are concerned with, in the interstellar medium (ISM) of galaxies is in general not known very well from direct observations. However from the observed near-linear correlation between metallicity and the dust-to-gas ratio (e.g. Issa et al. 1990; Lisenfeld \& Ferrara 1998; Draine et al. 2007; da Cunha et al. 2010, also see below) it is possible to conclude that it shows relatively little variation in nearby galaxies. Chemical evolution models do however show that $\xi$ is expected to show moderate evolution with time (e.g. Edmunds 2001; Inoue 2003; Calura et al. 2008), justifying our decision to keep it as a free parameter. Note that the dust-to-gas ratio, $\delta_{\mathrm{DGR}}=\xi Z$, is a derived parameter in this chosen parametrisation, we will return to this quantity in section 5.2 below.

In the Milky Way the depletion pattern in the ISM along certain specific lines of sight is, however, fairly wellknown. CL01 made slight modifications to the default depletion pattern of Cloudy which is based on Cowie \& Songaila (1986) and Jenkins (1987). The most recent in-depth discussion of depletion patterns is the careful study of Jenkins (2009) and while there are differences to previous works the overall agreement is reasonable - although unfortunately the most important elements for our needs, $\mathrm{C}$ and $\mathrm{O}$, are affected by rather significant systematic uncertainties (see the discussion in Jenkins 2009). The depletion patterns and abundances adopted by CL01 results in an average $\xi$ of 0.3 . 


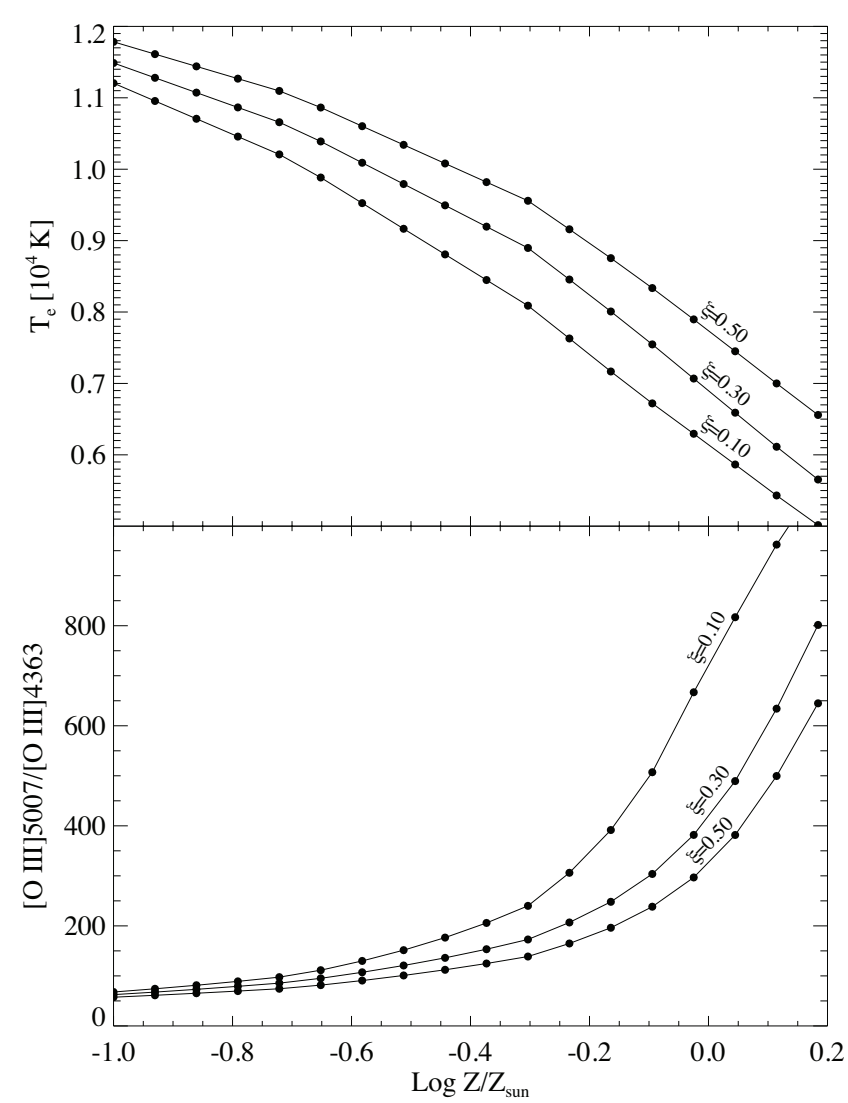

Figure 1. Top panel: The mean electron temperature as a function of metallicity for three different values of $\xi$. Note that as more metals are removed from the gas-phase, the mean temperature goes up because the cooling is reduced. Bottom panel: The ratio of the [O III] 5007 line to the auroral line, [O III] 4363, as a function of metallicity. Note that the change in temperature has a much larger effect at high metallicity than at low - this limits our ability to constrain $\xi$ at low metallicities. The curves in both plots were done at fixed ionisation parameter $(\log U=-3.4)$ and dust attenuation $\left(\tau_{V}=0.17\right)$.

This can be contrasted with Jenkins (2009) whose modelling implies a variation in $\xi$ from $\xi \sim 0.2$ to $\xi \sim 0.6$ along various lines of sight in the Milky Way with both a different depletion pattern and cosmic abundances than CL01. For reference, our $\xi=0.3$ corresponds to $F_{*} \approx 0.2$ in Jenkins' notation.

Keeping other parameters fixed, the main effect of changing $\xi$ is that removing more metals from the gas leads to an increase in the electron temperature in the $\mathrm{H}$ II-region. We show this explicitly for the CL01 models in Fig. 1 where the top panel shows the electron temperature as a function of total metallicity for three different values of $\xi$. As expected, a larger depletion (larger $\xi$ ) leads to a higher temperature at fixed metallicity.

The impact of this temperature change is not uniform in observable line ratios. The lower panel of the figure illustrates this with the well-known temperature sensitive line ratio $[\mathrm{O}$ III $] 5007 /[\mathrm{O}$ III $] 4363$ where we can see that the effect of the change in temperature is substantial at high metallicity, but much less noticeable at low metallicities.

An illustration of the effect of varying $\xi$ and $Z$ in the Baldwin et al. (1981, BPT) [N II]6584/H $\alpha$ versus
[O III $] 5007 / \mathrm{H} \beta$ diagnostic diagram, frequently used to classify galaxies based on their emission line properties (e.g. Kauffmann et al. 2003a; Kewlev et al. 2006) can be seen in the leftmost panel of Fig. 2 The distribution of emission line galaxies in the SDSS DR7 is shown as the underlying $2 \mathrm{D}$ histogram which is coloured according to the emission line classification, star-forming (purple), composite (light green) and active galactic nuclei (AGN, dark green). The middle panel in the figure shows the [S II] $6717 / \mathrm{H} \alpha$ versus [O III $] 5007 / \mathrm{H} \beta$ diagnostic diagram with the background $2 \mathrm{D}$ histogram again showing the distribution of SDSS DR7 galaxies with the colour indicating the mean classification based on the first diagram. The final panel shows the ionisation parameter sensitive ratio [O II] 3727/[O III] 5007 plotted against $[\mathrm{N} \mathrm{II}] 6584 / \mathrm{H} \alpha$.

The lines show the model tracks for fixed values of $\xi$, increasing from 0.1 to 0.5 from left to right. There are three sets of nine lines corresponding to $\log U=-2.8,-3.4,-3.9$ respectively (see the rightmost panel), going from the top downwards in all panels. The colouring of the lines correspond to a change in $\log Z$ from $0.1 Z_{\odot}$ for black to $4 Z_{\odot}$ for red. The effect of changing $\tau_{V}$ is not shown in this figure but is minor in the first two panels and should be well known.

As shown in Fig. 1 increasing $\xi$ increases the temperature, but it also reduces the amount of oxygen in the gas-phase. At high metallicity the resulting increase in the electron temperature, $T_{e}$, is more important than the reduced abundance of oxygen, thus increasing the flux in [O III]5007 relative to $\mathrm{H} \beta$, while nitrogen is assumed to be non-refractory and the increased $T_{e}$ leads to an increase in the $[\mathrm{N}$ II $] 6584 / \mathrm{H} \alpha$ and $[\mathrm{S}$ II $] 6717 / \mathrm{H} \alpha$ ratios (see also the discussion in CL01). This effect of $\xi$ on the emission lines is the reason why we are able to place some constraints on its value from observations. At low metallicity and high $T_{e}$ the effect of $\xi$ is weak leading to poor constraints on $\xi$, and we will return to this point below and in Appendix 3.1 In the first two panels the effect of the ionisation parameter is not easily isolated at high metallicity, but the final panel shows that the inclusion of [O II] 3727 helps separate the model tracks. This highlights the need to have a number of emission lines across the optical range to constrain the various model parameters. We also see that the model grid cover the emission-line properties of star-forming galaxies in the SDSS.

Given the CL01 model predictions we then adopt the Bayesian approach outlined in B04 (see also Kauffmann et al. 2003b) to derive the probability distribution functions (PDFs) for the parameters of interest. Given a particular set of lines, $\left\{L_{i}\right\}$, we use Bayes' theorem to calculate the log likelihood of each model, $\mathcal{M}\left(U, \xi, \tau_{V}, Z\right)$, as:

$$
\ln P\left(\mathcal{M} \mid\left\{L_{i}\right\}\right)=-\frac{1}{2} \sum_{i \in\left\{L_{i}\right\}} \frac{\left(f_{i}-A f_{\mathcal{M}}\right)^{2}}{\sigma_{i}^{2}}+\ln \operatorname{Pr}
$$

where $f_{i}$ is the flux in line $i, A$ is a scaling-factor and $f_{\mathcal{M}}$ corresponds to the relevant model. Pr denotes the prior on the model parameters and is shown in the top row of Fig. 3 , see also Appendix 3.1 and we have ignored the overall normalisation factor which is acceptable as long as the $\sigma_{i}$ 's do not depend on the model.

To calculate $\sigma_{i}$ for the SDSS galaxies we start with the 

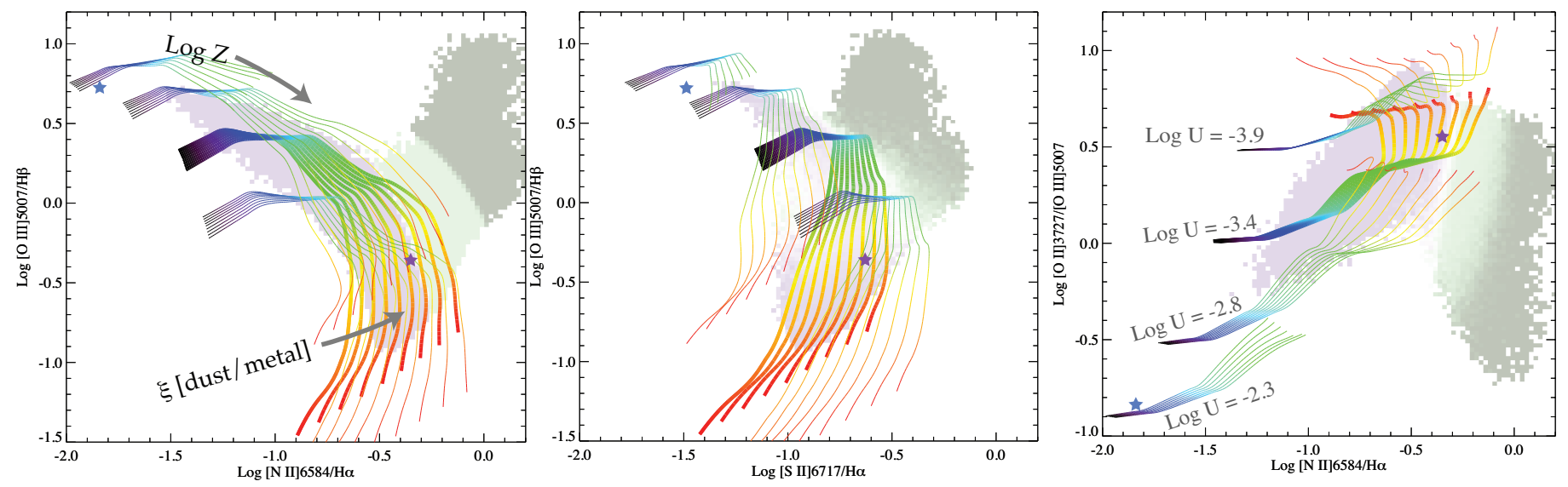

Figure 2. The behaviour of the CL01 models in three diagnostic diagrams. The background histogram shows the distribution of emission line galaxies in the SDSS DR7 and is coloured according to the mean emission line classification following B04 with star-forming (purple), composite (light green) and active galactic nuclei (AGN, dark green) classes shown. There are four groups of model tracks for different values of $\log U$ as indicated in the rightmost panel. The colour changes proportionally to $\log Z$ from $Z=0.1 Z_{\odot}$ in the top left to $Z=4 Z \odot$ at the bottom. For each $\log U$ value, except the highest, there are lines for nine $\xi$ values, linearly spaced from $\xi=0.1$ to $\xi=0.5$. The purple star shows the location of the object used for the illustrations of the fitting process in Figures 3 and 4 and the blue star that of Figure 5. The two leftmost panels show two BPT diagrams with slightly different sensitivity to $\xi$, while the rightmost panel plots $[\mathrm{O}$ II $] 3727 /[\mathrm{O} \mathrm{III}] 5007$ on the y-axis against $[\mathrm{N} \mathrm{II}] 6584 / \mathrm{H} \alpha$ to better separate the lines for different ionisation parameter.

$\begin{array}{lc}\text { Line } & \text { Scaling factor } \\ {[\mathrm{O} \text { II }] 3727} & 2.199 \\ \mathrm{H} \beta & 1.882 \\ {[\mathrm{O} \text { III }] 4959} & 1.573 \\ {[\mathrm{O} \text { III }] 5007} & 1.566 \\ \mathrm{H} \alpha & 2.473 \\ {[\mathrm{~N} \text { II }] 6584} & 2.039 \\ {[\mathrm{~S} \mathrm{II}] 6716,6731} & 1.621\end{array}$

Table 1. Factors to scale formal line flux errors by derived from a comparison of duplicate observations of SDSS galaxies.

formal line flux errors calculated from the SDSS error spectrum. These errors do not include continuum subtraction uncertainties and are therefore likely to underestimate the true uncertainties. To adjust for this we make use of duplicate observations of 33,794 emission line galaxies. For these we calculate the difference in measured line flux, normalised by the formal uncertainty of this difference. When the errors are accurately estimated the distribution of these differences should be a unit Gaussian and we use this to find the empirical scaling factors for the uncertainties that would bring the duplicate observations in agreement within the errors. These are given in Table1 For the other data sources we use published uncertainties. In both cases we furthermore follow B04 and apply an approximate theoretical uncertainty by adding 4 per cent of the line flux in quadrature to the uncertainties to get the final $\sigma_{i}$ that enters into equation (14). This theoretical error is an approximate adjustment to take into account the fact that each model is characterised by a single set of parameters while in reality each region likely has a distribution of physical properties (see B04 for more discussion).

To obtain constraints on a particular quantity we follow normal practice and marginalise $P$ onto the parameter in question. For the current discussion we are mostly interested in $\Sigma_{\text {gas }}$, and the probability in bin $i$ is given by
$P\left(\Sigma_{\text {gas }, i}\right)=\sum_{\mathcal{R}} P\left(\mathcal{M} \mid\left\{L_{i}\right\}\right)$,

where $\mathcal{R}$ is the region defined by

$$
\begin{gathered}
\mathcal{R}=\left\{\mathcal{M}\left(U, \xi, \tau_{V}, Z\right) \mid \log \left(\Sigma_{\text {gas }, i}-\delta_{\Sigma}\right)<\right. \\
\left.\log \frac{\tau_{V}}{\xi Z}<\log \left(\Sigma_{\text {gas }, i}+\delta_{\Sigma}\right)\right\} .
\end{gathered}
$$

We use a regular grid in $\log \Sigma_{\text {gas }}$ with bin width $\Delta_{\Sigma}=$ 0.05 dex between $\log \Sigma_{\text {gas }}=-1$ and $\log \Sigma_{\text {gas }}=4$ for the calculation of $\Sigma_{\text {gas }}$.

For each PDF we also calculate the entropy

$S=\sum_{i} P_{i} \log _{2} P_{i}$

where the sum is over the bins of the PDF. This is a generalised uncertainty estimate for the PDF and is useful when the PDF is multi-peaked, or for determining whether a distribution is significantly different from the prior distribution. For the latter question we also calculate the KullbackLeibler divergence between the prior, $Q_{i}$, and the final PDF, $P_{i}$,

$d_{\mathrm{KL}}=\sum_{i} P_{i} \log _{2}\left(\frac{P_{i}}{Q_{i}}\right)$.

Note that $d_{\mathrm{KL}}$ is often defined with natural logarithms rather than base-2 logarithms, but we choose the latter for consistency with the entropy definition.

The process is illustrated in Figures $3 \& 4$ where we show how the likelihood distribution of five different quantities changes as an increasing number of lines is included in the summation in equation (14). Between one row and the next we add the line indicated in the left-hand edge to the sum. The first row in the figure corresponds to the fit when only one line is included; this obviously provides no constraints so returns approximately the priors applied to the different quantities. From left to right the columns correspond to the total dust attenuation in the $V$-band, 


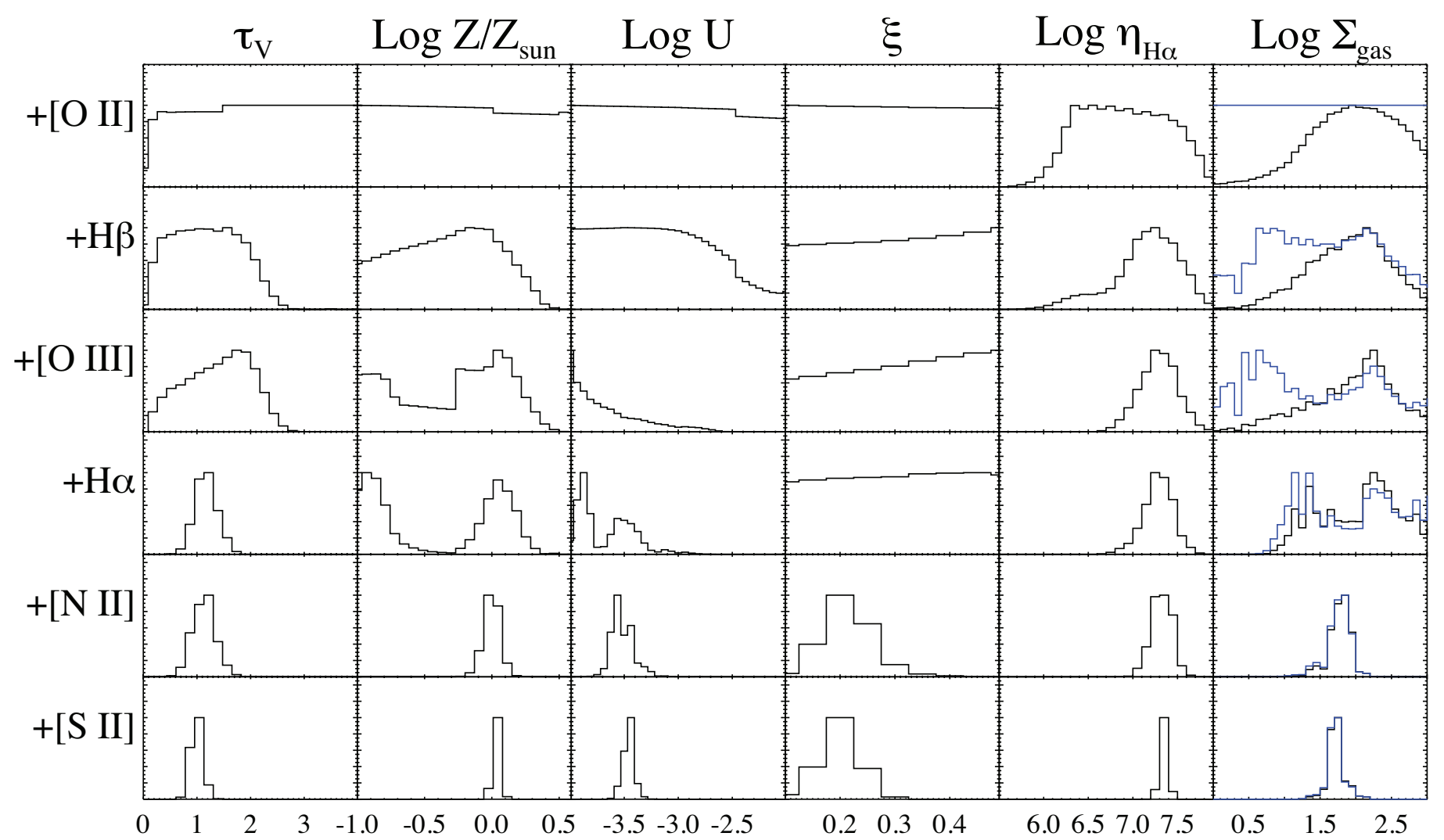

Figure 3. An example of the result of the fit to the spectrum given by plateID-MJD-fiberID=736-52221-287. The location of this object in the BPT diagram is indicated by the purple square in Figure 2 Each column shows the PDF for one derived parameter as emission lines are progressively included to the fit (equation 14). The leftmost column shows the constraints on the total $V$-band optical depth, $\tau_{V}$. The following three columns show the metallicity, the ionisation parameter, $\log U$ and the dust-to-metal ratio of the ionised gas, $\xi$. The second-to-last column shows the $\log$ of the conversion factor between $\mathrm{H} \alpha$ luminosity and star formation rate, $\eta_{\mathrm{H} \alpha}$, in units of $\mathrm{M}_{\odot} \mathrm{yr}^{-1} \mathrm{~L}_{\odot}^{-1}$. The final column shows $\log \Sigma_{\text {gas }}$ in units of $\mathrm{M}_{\odot} \mathrm{pc}^{-2}$. Given the construction of this plot, the top row essentially shows the prior adopted on the quantity in question in the model. In the last column we also show the PDF derived with a flat prior on $\Sigma_{\text {gas }}$ in blue. See the text for a discussion.

$\tau_{V}$, the metal abundance in units of solar metallicity, $\log Z$, the ionisation parameter, $\log U$, the dust-to-metal ratio of the ionised gas, $\xi$, the log of the conversion factor between $\mathrm{H} \alpha$-luminosity and star formation rate, $\eta_{\mathrm{H} \alpha}$ in units of $\mathrm{M}_{\odot} \mathrm{yr}^{-1} \mathrm{~L}_{\odot}^{-1}$ and finally the $\log$ of the gas column density, $\Sigma_{\text {gas }}$, in units of $\mathrm{M}_{\odot} \mathrm{pc}^{-2}$.

Focusing first on Fig. 3, in the first column we see the well-known fact that to get a good constraint on the dust attenuation at least two recombination lines must be available (here $\mathrm{H} \alpha$ and $\mathrm{H} \beta$ ). Some (minor) improvement on the attenuation estimate is possible if a better constraint on the electron temperature, and thence the intrinsic $\mathrm{H} \alpha / \mathrm{H} \beta$ Case $B$ ratio, can be had through the addition of [N II] 6584 and [S II]6717. Fig. 4] shows the same but with the lines added in the reverse order which illustrates the usefulness of $\mathrm{H} \beta$ and [O II] - for this particular object [O II] does not add much but $\mathrm{H} \beta$ is essential.

The second column shows the constraints on the metallicity - in this case this is very similar to what is obtained for the oxygen abundance, and shows the well-known fact (e.g. Pagel et al. 1980; McGaugh 1991) that an abundance indicator based on [O II]3727, [O III] 5007 and $\mathrm{H} \beta$ is doublevalued in some range of parameter space. The addition of
[N II] 6584 serves to break this degeneracy and we obtain a tight constraint on $Z$.

The ionisation parameter is shown in the third column and is only well-constrained in this case when a good dust correction can be obtained and lines with a range in ionisation potential are included. The dust-to-metal ratio of the ionised gas, $\xi$, is only well constrained when lines from non-depleted elements, such as $\mathrm{N}$ and $\mathrm{S}$, are included in the fit. Note that while CL01 assumed that S is not depleted in the ISM, it is not entirely clear that this is correct (e.g. Calura et al. 2009; Jenkins 2009, and references therein), it is however outside the scope of this paper to study this further. As should be clear from Fig. 2, the quality of the constraint on $\xi$ is a strong function of abundance and at a low abundance we are not able to place any constraints on $\xi$ at all. The conversion factor between $\mathrm{H} \alpha$-luminosity and SFR was discussed in CL01 and B04 and we refer the readers to those articles (note that the similarity between the PDF for $\log \eta_{\mathrm{H} \alpha}$ and $\log \Sigma_{\text {gas }}$ is not a general feature).

The PDF for $\Sigma_{\text {gas }}$ is shown in the last column. The first point to notice is that the prior on $\Sigma_{\text {gas }}$ is not flat. This is a natural consequence of it being a ratio of three quantities with flat priors. Thus we show the impact of the prior by also showing the PDF if one assumes a flat prior on $\Sigma_{\text {gas }}$ 


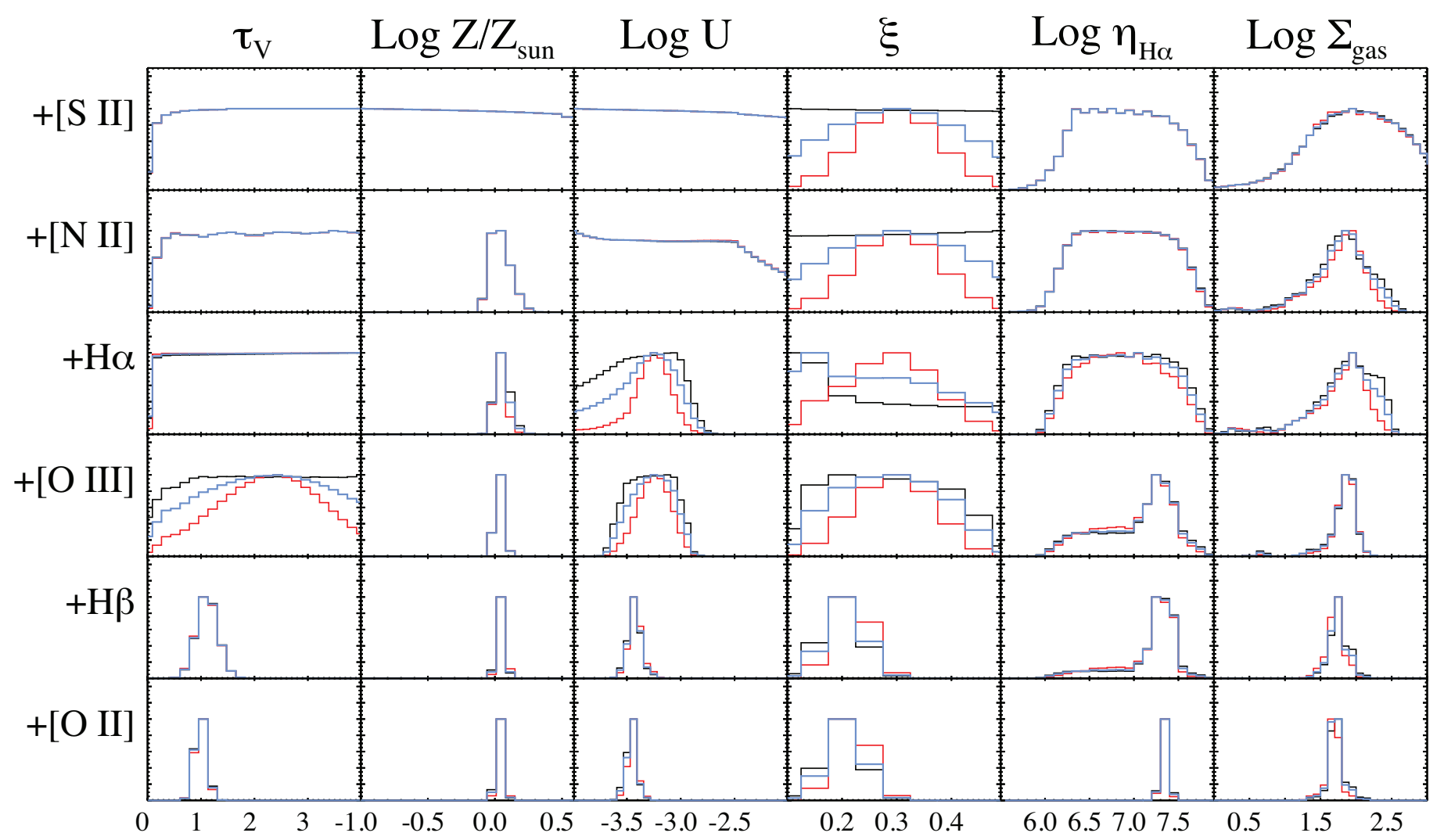

Figure 4. Similar to Fig. 3 but the emission lines are added in reverse order, providing a clearer illustration of the importance of $[\mathrm{O}$ II $] 3727$ and $\mathrm{H} \beta$ for the fitting procedure. The effect of applying a broad (blue) and narrow (red) prior on $\xi$ is also indicated here. Since this is a relatively metal rich galaxy, the effect is modest on most quantities as long as enough emission lines are present.

(blue line). This indicates that as long as the PDF is well constrained, the impact of the prior is minor.

This figure is for a "typical" galaxy in the SDSS and hence has close to solar metallicity. The figure is representative for such galaxies and the main points to note are: One of $[\mathrm{N}$ II] 6584 or $[\mathrm{S} \mathrm{II}] 6717$ should be available in addition to $\mathrm{H} \beta$, [O III] 5007 and $\mathrm{H} \alpha$ to break the abundance degeneracy. Fig. 4 shows the impact of lines in the reverse order and while in the shown case [O II] 3727 does not improve the constraints significantly in general it is beneficial to have this line as well.

At lower metallicity we can still constrain the column density of dust using the model above, but as we are unable to place strong constraints on $\xi$, our $\Sigma_{\text {gas }}$ estimates have larger error-bars and we are somewhat sensitive to the prior placed on $\xi$, thus we turn to look at this next.

\subsection{The impact of priors on $\xi$ on the $\Sigma_{\text {gas }}$ determinations}

An advantage of the Bayesian approach to parameter estimation is that it makes it necessary to state prior beliefs explicitly. In general the priors adopted will not be important if the evidence outweighs the prior belief. However for quantities that are not well constrained by the evidence, the derived constraints are usually affected by the priors adopted.

By default the model parameters have flat priors over the range covered by the models. Note that this does of course imply a preferred median value in the case of no information which is set by the mid-point of the range covered by the parameters. However as long as most of the optical lines are present with good $\mathrm{S} / \mathrm{N}$ this is unimportant and most quantities we are interested in, such as the oxygen abundance, $\mathrm{O} / \mathrm{H}$, the star formation rate, $\mathrm{SFR}$, and the dust attenuation, $\tau_{V}$ are unaffected by the choice of prior - at least as long as we do not adopt strongly peaked priors on the model parameters.

This is not always the case for $\xi$. This parameter has a more subtle effect on emission line fluxes and can only be reliably determined at high metallicity where its impact on cooling is significant (c.f. Fig. 1). At low metallicity it is not possible to put strong constraints on $\xi$ and its derived value is thus sensitive to the prior adopted.

Since $\xi Z$ provides a constraint on the dust-to-gas ratio that we need to convert dust column density to gas column density, it is natural to ask how sensitive $\Sigma_{\text {gas }}$ is to the adopted priors. In Fig. 3] we show one example where adopting a flat prior on $\Sigma_{\text {gas }}$ for a metal rich system made little difference on the final result, but for a general study it is better to focus our attention on $\xi$.

To explore this dependence we therefore explore three different priors for $\xi$. The default prior is flat between $\xi=$ 0.1 and $\xi=0.5$, and then we have two priors taken to be Gaussians in $\xi$. We centre both on $\xi=0.3$ because, as we mentioned above, this is the average value for the SDSS 


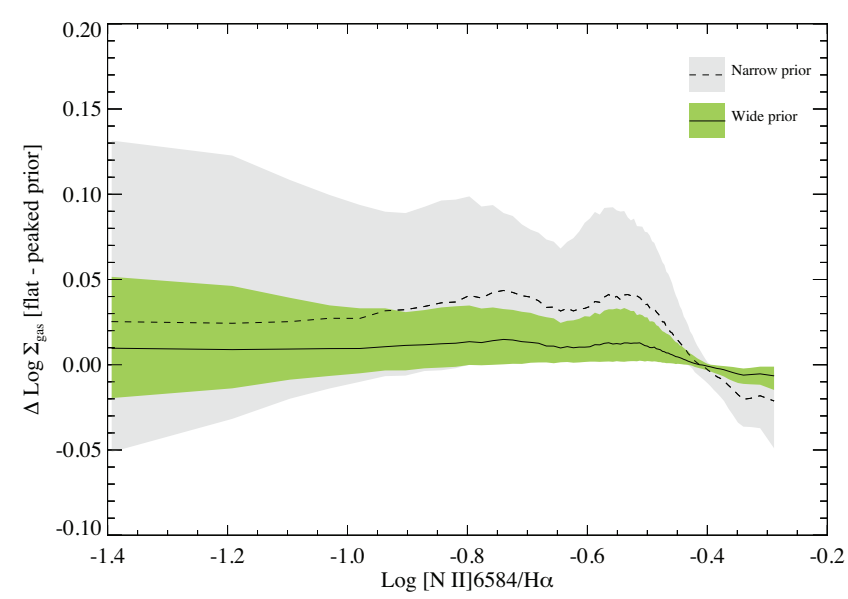

Figure 6. The figure shows the effect on our estimates of $\Sigma_{\text {gas }}$ for all star-forming galaxies in the SDSS DR7 from changing the prior on $\xi$ from flat to a Gaussian with width $\sigma=0.08$ (grey shading/dashed line) or to a Gaussian with width $\sigma=0.15$ (green shading/solid line). The dashed and solid lines show the median trend while the shaded area encloses 68 per cent of the scatter.

and also in good agreement with theoretical models when adjusted to the same abundances as used in the CL01 models (e.g. Edmunds 2001; Calura et al. 2008). For the wide prior we take a width of $\sigma_{\xi}=0.15$ while the narrow prior has $\sigma_{\xi}=0.08$.

Fig. 5 compares the results of fitting with a flat prior on $\xi$ (black line) to that obtained with the narrow prior on $\xi$ (red line) and that of a broad prior (blue line). The object is a fairly low-metallicity galaxy $(12+\log \mathrm{O} / \mathrm{H} \approx 7.8)$ so that the constraints on $\xi$ are weak, the format is otherwise the same as in Fig. 3 It is quite clear from the figure that the effect of changing the prior on $\xi$ is negligible for most parameters, but using a peaked prior on $\xi$ does lead to somewhat tighter constraints on $\Sigma_{\text {gas }}$. This improvement in the constraints is of course prior dependent, thus the most crucial question is whether changing the prior leads to systematic differences in the gas mass estimates.

Fig. 6] shows that the effect of the prior on $\xi$ on the gas mass estimates is modest. The figure shows the difference between $\Sigma_{\text {gas }}$ estimated using a flat prior and that obtained using a peaked prior. The dashed line shows the median trend for the narrow prior and we plot it against the [N II $] 6584 / \mathrm{H} \alpha$ ratio as an approximate proxy for metallicity. We can see that the difference is typically less that 0.05 dex even for the most peaked prior on $\xi$. When viewed as relative changes, we find that even at the lowest metallicities less than 5 per cent of the galaxies show changes in excess of 30 per cent.

It is important to note that the choice of prior also has an effect at high metallicity. This is because although at high metallicity we can constrain the value of $\xi$, applying a peaked prior on $\xi$ will bias its estimate towards the peak of the prior, with an effect that is stronger for a more peaked prior, as seen in the figure, because stronger evidence is then required to overcome the prior belief. Figure 4 shows, however, that as long as the emission lines are detected at sufficiently high signal-to-noise, the effect of a prior on $\xi$ is very modest at high metallicity. Thus employing a $\xi$-prior primarily makes sense in low-metallicity systems, at high metallicity there is enough information to constrain $\xi$ independently and applying a peaked prior could bias the estimate of $\xi$ and $\Sigma_{\text {gas }}$.

While we do not show it explicitly here, the effect of a prior on $\xi$ on other parameters is very small, at least at low metallicity. The oxygen abundance, $12+\log \mathrm{O} / \mathrm{H}$, for instance, changes by less than 1 per cent when applying a narrow prior on $\xi$. Thus while applying a prior on $\xi$ does lead to slight, but systematic, offsets in our $\Sigma_{\text {gas }}$ estimates, at least these are independent of other parameters to first order. We have however, not discussed in detail what an appropriate prior should be - the simplest choice is indeed a prior that is metallicity independent but as we will see later, in section 5.1 it might be better to implement a metallicity dependent prior.

\subsection{The importance of the attenuation curve}

Equation (13) depends on the absolute value of the V-band optical depth, which implies a dependence on the attenuation curve adopted. While the shape of the optical attenuation curve is more constrained than, say, the UV, it must still be treated as an uncertain ingredient in the models.

For a simple attenuation curve $\tau(\lambda) \propto \lambda^{-n}$, we have

$\tau_{V}=\frac{\lambda_{V}^{-n}}{\lambda_{\mathrm{H} \beta}^{-n}-\lambda_{\mathrm{H} \alpha}^{-n}} \ln r$

where $r$ is the ratio of the Balmer decrement to the Case B ratio. Thus a change from $n=-0.7$ to $n=-1.3$ leads to an change in $\log \Sigma_{\text {gas }}$ of -0.28 for the same Balmer ratio and corresponding changes to the SFR estimate. When applying the fitting methodology described above, the final differences are slightly different because of slight changes in the bestfit model. Overall however, parameters that do not depend directly on $\tau_{V}$, such as $\mathrm{O} / \mathrm{H}, \xi$, and $\log U$, are very weakly affected by a change in $n$.

More complex attenuation laws do not in general lead to constant offsets. We therefore have therefore derived estimates of $\log \Sigma_{\text {gas }}$ for four different attenuation laws as follows:

(i) $\tau(\lambda) \propto \lambda^{-1.3}$. CF00 observed that a slope of -1.3 corresponds to the middle range of the optical properties of dust grains between the Milky Way, the Large and the Small Magellanic Cloud. This was argued to be appropriate for attenuation of birth clouds in da Cunha et al. (2008) and Wild et al. (2011). We adopt this as our reference law.

(ii) $\tau(\lambda) \propto \lambda^{-0.7}$. This is the attenuation law recommended by CF00 for absorption ultraviolet to infrared continua by the ambient ISM in star-forming galaxies, and used in e.g. Brinchmann et al. (2004). However the attenuation of emission lines is likely less grey than this.

(iii) $\tau(\lambda) / \tau\left(\lambda_{V}\right)=(1-\mu)\left(\lambda / \lambda_{V}\right)^{-1.3}+\mu\left(\lambda / \lambda_{V}\right)^{-0.7}$. This is the combination of (1) and (2), as suggested observationally by Wild et al. (2007) and motivated theoretically by da Cunha et al. (2008). $\mu$ here is the relative contribution to the total $\tau_{V}$ contributed by the ambient ISM. A value of $\mu=0.3$ was suggested by CF00, but it is also possible to fit this keeping $\mu$ a free parameter.

(iv) $\tau(\lambda) / \tau\left(\lambda_{V}\right)=(1-\mu)\left(\lambda / \lambda_{V}\right)^{-1.3}+\mu \lambda^{-n(\lambda)}$ with $n(\lambda)=2.8 /\left(1+3 \sqrt{\mu \tau_{V}}\right)+\left(0.3-0.05 \mu \tau_{V}\right)(\lambda-0.55)$, with $\lambda$ measured in microns. This is the effective attenuation curve 


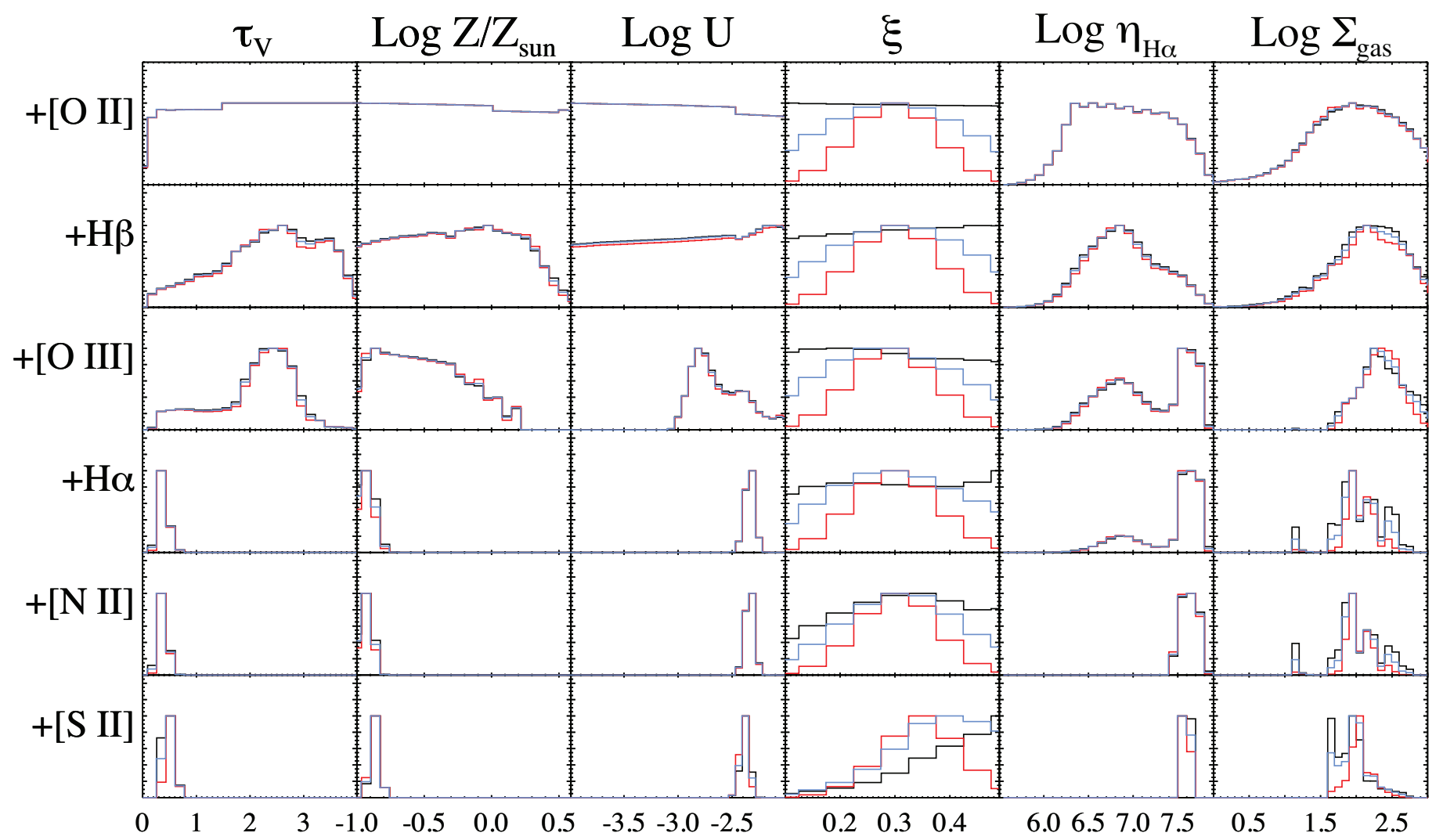

Figure 5. Similar to Fig. 3 but this time showing a low metallicity galaxy (691-52199-0636 at $z=0.038)$. The results with a flat prior on $\xi$ is shown in black, overplotted on this in red we show the results for the narrow prior on $\xi$ and in blue that of a broad prior on $\xi$. The effect of the prior is striking for $\xi$ but rather modest for everything else.

Table 2. Sections where the influence of non-standard priors is explored throughout the paper

Flat prior on $\Sigma_{\text {gas }}$ versus the default peaked prior on $\Sigma_{\text {gas }}$ The effect of peaked priors on $\xi$ relative to the default flat prior

The impact of changing the dust attenuation laws

The importance of a variable $X_{\mathrm{CO}}$ on the tests of the method
Figures 3 and 4

Sections 3.1 and 5.3

Results in section 6 are checked

for sensitivity to the choice of $\xi$ prior.

Section 3.2

All figures use $\tau(\lambda) \propto \lambda^{-1.3}$

The comparisons in section 5.3 use

$X_{\mathrm{CO}}$ from equation (23) recommended by Chevallard et al (2013, submitted) based on an analysis of a range of radiative transfer models.

These all give broadly speaking the same $\log \Sigma_{\text {gas }}$ estimates, but with systematic shifts between the determinations. To make this quantitative, we fit all SDSS DR7 starforming galaxies with each of the four different attenuation curves. The median effect is such that with option 2 above, $\log \Sigma_{\text {gas }}$ is increased by 0.30 dex in the mean relative to our reference curve, while option 3 leads to $\log \Sigma_{\text {gas }}$ estimates higher by 0.19 dex in the mean than the reference values. The fourth option produces gas column densities that are 0.06 dex higher than our reference value.

In the following we will adopt option 1 above as it is a simple law and it is also very similar to the widely used Seaton (1979) extinction curve at $\lambda>4200 \AA$. Furthermore option 1 gives results that are very similar to that of option
4 which is the most rigorously justified curve, but which depend on one further parameter, $\mu$, which is not well constrained by emission lines alone.

\subsection{The importance of the abundance estimator}

It is well-known that different abundance estimators can give significantly different oxygen estimates for the same $\mathrm{H}$ II regions (see Kewley \& Ellison 2008, for a comparison). Since our estimator depends on the absolute value of the metallicity, we are affected by this uncertainty and it is therefore appropriate to revisit this issue for our technique.

Abundance comparisons are generally made between $d i$ rect estimators where auroral lines are used to determine electron temperatures appropriate for different ionisation stages of elements (e.g. Izotov et al. 2006) and other techniques. The basic assumption is that the abundances pro- 


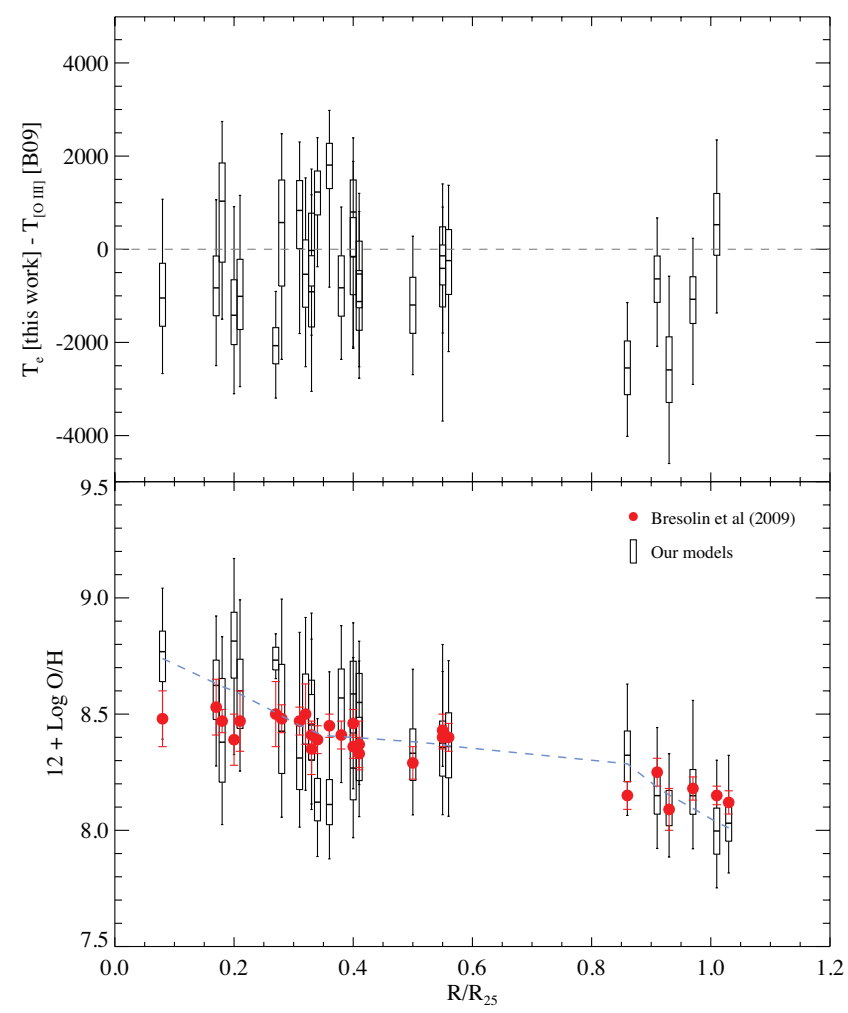

Figure 7. Top panel: The difference in effective $T_{e}$ from our fits and the electron temperature in the [O III] zone from the direct method calculation by Bresolin et al. (2009). The agreement is satisfactory given that our fits do not include any particularly temperature sensitive lines. Bottom panel: The abundance as a function of the radius normalised by $R_{25}$ for NGC 300 . The solid red circles show the oxygen abundance derived in Bresolin et al. (2009) using the temperature sensitive method. The box plots show the oxygen abundances estimated using our model fits, using only the $[\mathrm{O} \mathrm{II}] 3727, \mathrm{H} \beta,[\mathrm{O} \mathrm{III}] 5007, \mathrm{H} \alpha$, and $[\mathrm{N} \mathrm{II}] 6584$ lines in the fit. The horizontal line shows the median and the box encloses 50 per cent of the likelihood while the error bars enclose 95 per cent of the likelihood. The blue dashed line is a local regression, or loess, smooth of the medians of our estimates.

vided by the direct method are the most reliable and thus provide a reference abundance estimate.

For our comparison here we wish to extend this comparison to as high metallicity as possible. To do so, we took the comprehensive study of NGC 300 by Bresolin et al. (2009) who published line fluxes for $\mathrm{H}$ II regions across the galaxy as well as abundances derived using the direct method. We removed the de-reddening applied to the published fluxes and ran our models. The resulting comparison of oxygen abundance estimates is shown in Fig. 7

The top panel compares the mean electron temperature in our models to that estimated from the direct method by Bresolin et al against the radius in units of $R_{25}$. The difference between the $T_{e}$ predictions from our models and the published $T_{\text {[OIII] }}$ estimates, including the published uncertainty on the $T_{[\mathrm{OIII}]}$ values, are shown as box-plots where the horizontal line shows the median, the boxes enclose 50 per cent of the likelihood, and the vertical bars enclose 95 per cent, or $\pm 2 \sigma$ for Gaussian errors. The agreement is quite satisfactory, in particular given that our fits, in contrast to the direct method, do not include any lines that are strongly sensitive to $T_{e}$, such as [O III] 4363 .

In the bottom panel we show the resulting oxygen abundance gradient. The solid circles show the data from Bresolin et al, while the box plots show the results of our fits, with the dashed line indicating a local regression, or loess, smooth of the medians from our fits. The immediate point to notice is that the abundances are in very good agreement at nearly all radii - this is in contrast to the comparisons in Bresolin et al. (2009) which used fitting formulae for the strong line abundance estimates.

In general we see similar agreement at lower metallicities although there has been some indication of a poorer fit in systems with significantly enhanced nitrogen relative to oxygen (Yin et al. 2007). This is likely caused by the fixed abundance ratios adopted at a given metallicity in the current CL01 models. These systems typically have a very high $\mathrm{SFR} / \mathrm{M}_{*}$ and make up only a very small fraction of the sample used in the present work.

Most of our galaxies are more metal rich than the range probed in Fig. 7 so it is natural to ask whether the agreement continues or not. This is uncertain but here we will take the approach that we will a) test our gas mass estimates and look for offsets there and b) make the reasonable assumption that although there might be uncertainties in the absolute abundances, the relative abundances should be robustly determined. In passing we note that this uncertainty also leads to uncertainties in the determination of $\mathrm{H}_{2}$ masses due to the metal dependence of the $X_{\mathrm{CO}}$ conversion factor. Indeed with current parametrisations of this dependency, e.g. equations (23) and (24) below, the uncertainty on molecular masses due to metallicity uncertainties is as large, or larger than those intrinsic to our method.

\section{DATA USED}

In the next section we will discuss the tests we have made to verify the present method for gas column density and dustto-gas ratio estimation. To carry out these tests we need both optical spectroscopy to run our method on, as well as resolved and integrated gas mass measurements from $\mathrm{CO}$ and $\mathrm{H}$ I observations to compare our results with.

We use optical spectroscopy from the Sloan Digital Sky Survey (SDSS York et al. 2000) Data Release t1 (DR7 Abazaiian et al. 2009). The SDSS obtained 5-band imaging using a drift-scanning camera (Gunn et al. 1998) on the 2.5m telescope at Apache Point (Gunn et al. 2006). Spectroscopic targets were selected on the basis of the imaging and a tiling algorithm described in Blanton et al. (2003). The spectra were obtained using a fibre spectrograph with 640 fibres per plate, with 3" fibre apertures. The photometric system is discussed by Fukugita et al. (1996) with the photometric calibration being covered in more detail in Smith et al. (2002). Where relevant we use the photometry from the Catalogue Archive Server2. We classify galaxies into six classes following B04 based on their emission line properties. In particular we classify galaxies with $\mathrm{S} / \mathrm{N}>3$ in

\footnotetext{
1 http://www.sdss.org/dr7

2 http://cas.sdss.org/dr7/en
} 
$\mathrm{H} \beta$, [O III] $5007, \mathrm{H} \alpha$ and [N II]6584 into star-forming, composite and AGN based in the leftmost diagnostic diagram in Figure 2. In the following we will focus on the star-forming (SF) class.

We will also make use of the massive Integral Field Spectroscopy (IFS) mosaic of NGC 628 published by Sánchez et al. (2011) as part of the PINGS project 3 . This was obtained with the PPAK spectrograph on the $3.5 \mathrm{~m}$ telescope at Calar Alto. We use the reduced data provided on the PINGS project page and selected a total of 87 regions across the galaxy (see Fig. 8). For each region we sum up line fluxes within circular apertures of diameters of 6" to 24 ", we used the range of aperture sizes to test the sensitivity of our results to aperture size, but as the results are only weakly sensitive to aperture size we will focus on results for 12 " apertures in this paper unless otherwise stated. To ensure we have a consistent alignment of the images, we tie the IFU mosaic world coordinate system to that of the $\mathrm{H} \alpha$ image of NGC 628 provided by the SINGS survey (Kennicutt et al. 2003).

We will also make use of resolved H I maps from the THINGS survey (Walter et al. 2008) as well as CO maps from the BIMA SONG4 (Helfer et al. 2003), Nobeyama (Kuno et al. 2007) and HERACLES (Lerov et al. 2009b) surveys - we use HERACLES data whenever possible but use the other data sets for reference.

We match these datasets to the SDSS to find regions where we have resolved $\mathrm{HI}$ and (optionally) CO maps as well as SDSS spectra which we can use to obtain total gas column densities using our method. We searched the SDSS for all RC3 galaxies with multiple SDSS spectra across the face of the galaxy and then matched this to the THINGS and HERACLES surveys. This resulted in the identification of 100 SDSS spectra in 12 galaxies, see Table 3 for an overview of these. All these galaxies have H I maps from the THINGS survey and CO maps from the HERACLES survey, crosschecked against the BIMA SONG and Nobeyama surveys when possible. For these maps we also measure gas surface mass densities in apertures with diameters of 6", 12" and 24 "', but the spectroscopic aperture is of course fixed to 3" by the SDSS fibre diameter.

We will also make use of integrated $\mathrm{HI}$ data and for this we make use of the ALFALFA survey $\alpha$-40 release (Havnes et al. 2011), the second data release (DR2) for the GASS survey (Catinella et al. 2012) and the compilation of H I data by Springob et al. (2005, hereafter S05). For GASS DR2 we use the catalogues provided by Catinella et al. (2012), and for ALFALFA we use the match to SDSS DR7 provided by Haynes et al. (2011). We match S05 to the SDSS DR7 using a 5 arcseconds match radius, which is small but our aim is not to be complete here. We furthermore require that the velocity offset between the SDSS and $\mathrm{H}$ I redshifts is less than $250 \mathrm{~km} \mathrm{~s}^{-1}$. When there are $\mathrm{H}$ i observations from more than one survey, our order of priority is GASS, AL-

\footnotetext{
3 http://www.ast.cam.ac.uk/ioa/research/pings/html/index.html

4 Downloaded from

http://nedwww.ipac.caltech.edu/level5/March02/SONG/SONG.html

5 Downloaded from

http://www.nro.nao.ac.jp/ñro45mrt/COatlas/

6 Downloaded from

http://www.cv.nrao.edu/ãleroy/HERACLES/Overview.html
}

FALFA and S05. In total this gives 11,253 unique matches, 416 from GASS, 9,762 from ALFALFA and 1,259 from S05.

We calculate H I masses in solar masses from the fluxes using

$$
M_{\mathrm{H} \text { I }}\left[\mathrm{M}_{\odot}\right]=2.36 \times 10^{5} D^{2} S
$$

where $S$ is the flux in Jy, or Jy/beam for resolved data, and $D$ is the distance in Mpc. For the S05 dataset, which is abundant in edge-on galaxies, we adopt the flux corrected for self-absorption, while for the other datasets we use the observed flux. Note that we do not correct for inclination effects because these would affect the $\Sigma_{\text {gas }}$ derived from our method in the same way as it would the $\mathrm{H}$ I and CO maps. Thus throughout we will quote observed surface mass densities, not deprojected.

For the molecular gas data, we follow Leroy et al. (2008) and calculate molecular surface mass density maps from $\mathrm{CO}$ $J=1 \rightarrow 0$ maps using

$\Sigma_{\mathrm{H}_{2}}\left[\mathrm{M}_{\odot} \mathrm{pc}^{-2}\right]=4.4 \frac{X_{\mathrm{CO}}}{2 \times 10^{20}} I_{\mathrm{CO}}$,

with $I_{\mathrm{CO}}$ being the $\mathrm{CO}$ intensity in $\mathrm{K} \mathrm{km} \mathrm{s}^{-1}$. We convert the HERACLES $J=2 \rightarrow 1$ maps to $J=1 \rightarrow 0$ maps using the conversion $I_{\mathrm{CO}}(2 \rightarrow 1)=0.8 I_{\mathrm{CO}}(1 \rightarrow 0)$ recommended by Leroy et al. (2008). $X_{\mathrm{CO}}$ is the CO-to- $\mathrm{H}_{2}$ column density conversion factor in units of $\mathrm{cm}^{-2} \mathrm{~K}^{-1} \mathrm{~km}^{-1} \mathrm{~s}$ (see for instance Leroy et al. 2008). For Milky Way conditions it appears that a constant value of $X_{\mathrm{CO}}=$ $2 \times 10^{20} \mathrm{~cm}^{-2} \mathrm{~K}^{-1} \mathrm{~km}^{-1} \mathrm{~s}$ is appropriate (e.g. Dame et al. 2001), but as the galaxies considered here span a range in physical properties it is likely incorrect to adopt a fixed $X_{\mathrm{CO}}$ (e.g. Arimoto et al. 1996; Israel 1997b). Hence we also make use of a variable $X_{\mathrm{CO}}$. For this we use the metallicity dependent $X_{\mathrm{CO}}$ from Boselli et al. (2002) as our reference parametrisation,

$\log X_{\mathrm{CO}}=-1.01 \log \mathrm{O} / \mathrm{H}+29.28$,

but we have also explored the stronger trend advocated by Israel (1997b) viz.

$\log X_{\mathrm{CO}}=-2.6 \log \mathrm{O} / \mathrm{H}+11.6$.

Calibrations of $X_{\mathrm{CO}}$ against metallicity in the literature show a large variation between different authors (see Bolatto et al. 2008; Lerov et al. 2011; Kennicutt \& Evans 2012, for summaries). They range from finding no trend with metallicity, ie. a constant $X_{\mathrm{CO}}$ via a logarithmic slope of -1 to -2.6 . As Bolatto et al. (2008) points out, there are systematic variations between the techniques used to infer $X_{\mathrm{CO}}$ but the implication is that one must view $X_{\mathrm{CO}}$ as being significantly uncertain at low metallicity and a similar behaviour is also found at $z>1$ (Genzel et al. 2012). We remark that although the majority of the effort lately has focused on the metallicity dependence of $X_{\mathrm{CO}}$, it is possible that other factors, such as the intensity of the radiation field also could have an effect (e.g. Israel 1997b) but we ignore this complication here.

\section{TESTS OF THE METHODOLOGY}

We will test the method outlined in section 2 in two steps that logically follow from viewing equation (13) as having 

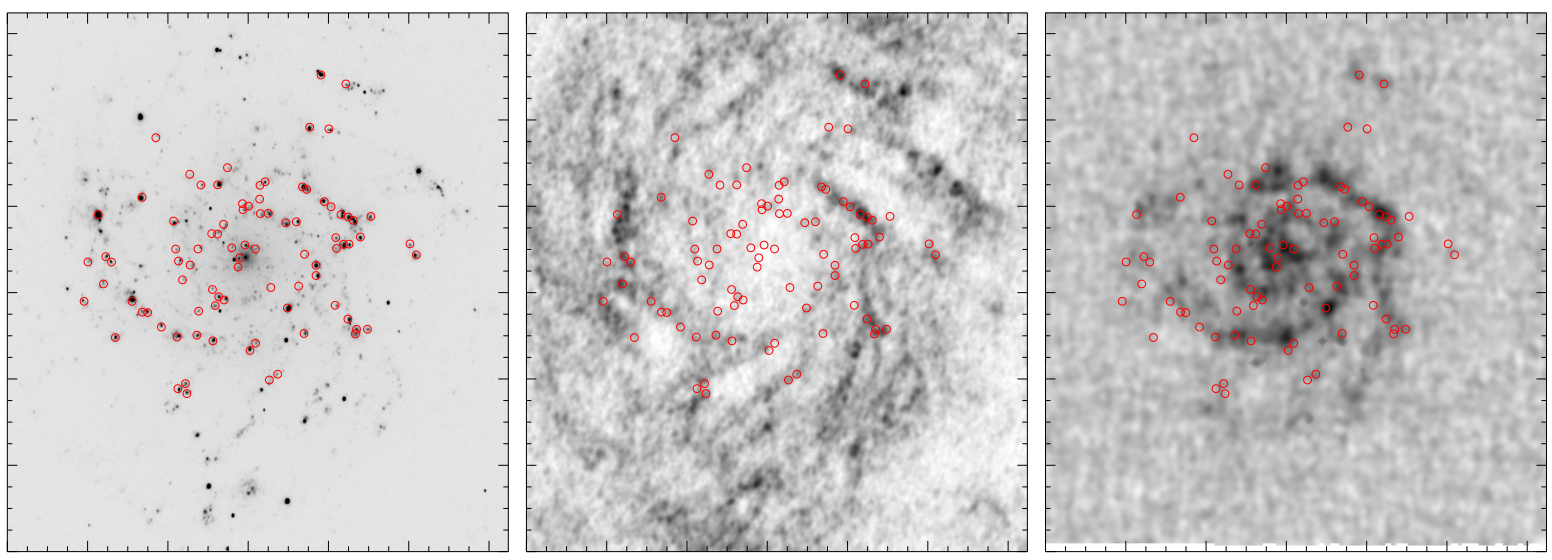

Figure 8. Three images of NGC 628, on the left the SINGS H $\alpha$ image, in the middle the H I image from the THINGS survey, and on the right the CO image from the HERACLES survey. The locations of the 87 regions in NGC 628 where we calculate gas column densities from the IFU, $\mathrm{HI}$ and $\mathrm{CO}$ maps are overplotted as red circles. Note that the regions sample a range of $\mathrm{HI} / \mathrm{CO}$ ratios.

Table 3. An overview of regions with $\mathrm{H}$ I and CO interferometric data, while at the same time have spectroscopy from SDSS or from the NGC 628 IFU mosaic consistent with being ionised by stars. The complete table is provided electronically. The first column gives the name of the galaxy with a running index for the region appended. The second column gives the SDSS spectroscopic identification, followed by the position of the fibre the spectrum was obtained through. The final column gives the zero-offset index of this object in the MPA-JHU value-added catalogues(Brinchmann et al. 2004) found at http//www.mpa-garching.mpg.de/SDSS.

\begin{tabular}{lcccc}
\hline Galaxy & PlateID-MJD-FiberID & \multicolumn{2}{c}{ Ra (J2000) Dec } & IDR7 \\
\hline NGC2403-00 & $2944-54523-237$ & $+07: 36: 51.23$ & $+65: 41: 01.65$ & 920120 \\
NGC2903-00 & $2289-53708-615$ & $+09: 32: 10.09$ & $+21: 30: 08.26$ & 794801 \\
NGC2903-01 & $2292-53713-310$ & $+09: 32: 09.68$ & $+21: 31: 06.60$ & 795658 \\
NGC2903-02 & $2289-53708-616$ & $+09: 32: 00.35$ & $+21: 27: 40.40$ & 794802 \\
NGC2976-00 & $1879-54478-291$ & $+09: 47: 15.31$ & $+67: 55: 00.05$ & 664806 \\
NGC2976-02 & $1878-54474-601$ & $+09: 47: 07.99$ & $+67: 55: 53.14$ & 664573 \\
NGC2976-03 & $1879-54478-281$ & $+09: 47: 07.99$ & $+67: 55: 53.14$ & 664798
\end{tabular}

two components: The dust column density and the dust-togas ratio, obtained through a combination of the metallicity and the dust-to-metal ratio, of the gas. We commence our testing by checking our dust-to-metal and dust-to-gas ratio estimates. We do not test our metallicity estimates explicitly here, see section 3.3 for a brief discussion of this. To test the gas column densities we take three different approaches that have different inherent systematic uncertainties and by combining these we should be able to provide a robust test of our method. The first technique compares $\Sigma_{\text {gas }}$ determined from SDSS spectra and the NGC 628 IFU mosaic with that of $\mathrm{HI}+\mathrm{CO}$ gas maps. The beams of the resolved maps are only larger than the SDSS fibres by a factor of $2-3$ so we anticipate only moderate aperture effects in this comparison. We will find below that our technique gives gas mass estimates that are in very good agreement with the $\mathrm{H}+\mathrm{CO}$ maps.

However the derivation in section 2 is only strictly valid for angle-averaged quantities, not resolved properties as used in this comparison. Furthermore, the sample for which we have good quality $\mathrm{H} \mathrm{I}$ and $\mathrm{CO}$ maps is relatively modest in size, and the galaxies are fairly close to face-on. This leads us to the second test which looks at how our gas estimates change as the aperture sampled changes - we will show that this way we can recover trends of $\mathrm{H}$ I content in galaxies found in recent $\mathrm{H}$ I surveys when the SDSS fibre samples a significant fraction of the stellar disk.

The natural final step is to compare our gas content measurements from SDSS spectroscopy to measurments of total H I masses which are available for large samples of galaxies today. This requires careful adjustments for the difference in apertures and it turns out that although this comparison works well, systematic uncertainties in the aperture corrections weaken any conclusion reached with this test. This comparison is therefore discussed in Appendix A

Note that in the following we will also define

$r_{\text {gas }}=\frac{\Sigma_{\text {gas }}}{\Sigma_{*}} \approx \frac{M_{\text {gas }}}{M_{*}}$,

as the gas ratio. Note that the second equality which we generally will assume holds, assumes that the scale-lengths of the gas and the stars are at least comparable. This is closely related to the gas fraction,

$f_{\text {gas }}=\frac{\Sigma_{\text {gas }}}{\Sigma_{\text {gas }}+\Sigma_{*}}=\frac{1}{1+1 / r_{\text {gas }}}$. 
Table 4. Measurements for the regions indicated in Table 3 the complete table is provided electronically.

\begin{tabular}{|c|c|c|c|c|c|c|c|c|c|c|}
\hline Galaxy & $\log \Sigma_{\mathrm{HI}}$ & $\begin{array}{c}\log \Sigma_{\text {mol }} \\
16 \%\end{array}$ & $\begin{array}{c}\log \Sigma_{\text {spec }} \\
\text { median }\end{array}$ & $\begin{array}{c}\log \Sigma_{\text {spec }} \\
84 \%\end{array}$ & $\begin{array}{c}\log \sum_{\text {spec }} \\
\AA\end{array}$ & $\begin{array}{c}\mathrm{EW}(\mathrm{H} \alpha) \\
\AA\end{array}$ & $\mathrm{EW}(\mathrm{H} \beta)$ & $\mathrm{H} \alpha / \mathrm{H} \beta$ & $g-r$ & $u-r$ \\
\hline NGC2403-00 & 1.07 & 1.07 & 0.59 & 1.25 & 1.92 & 17.9 & 3.1 & 3.19 & 0.16 & 0.99 \\
\hline NGC2903-00 & 0.58 & 2.00 & 1.09 & 1.21 & 1.31 & 73.0 & 13.2 & 5.36 & 0.65 & 1.58 \\
\hline NGC2903-01 & 1.21 & 1.53 & 1.07 & 1.11 & 1.18 & 164.7 & 21.0 & 5.81 & 0.49 & 0.94 \\
\hline NGC2903-02 & 0.93 & 0.95 & 0.96 & 1.29 & 1.54 & 44.6 & 10.8 & 3.51 & 0.21 & 0.64 \\
\hline NGC2976-00 & 0.87 & 1.45 & 0.93 & 1.04 & 1.15 & 58.1 & 15.8 & 3.81 & 0.91 & 2.21 \\
\hline NGC2976-02 & 1.33 & 1.87 & 1.61 & 1.78 & 1.91 & 207.9 & 32.3 & 4.24 & 0.47 & 0.94 \\
\hline NGC2976-03 & 1.33 & 1.87 & 1.67 & 1.80 & 1.91 & 178.6 & 27.3 & 4.22 & 0.47 & 0.94 \\
\hline
\end{tabular}

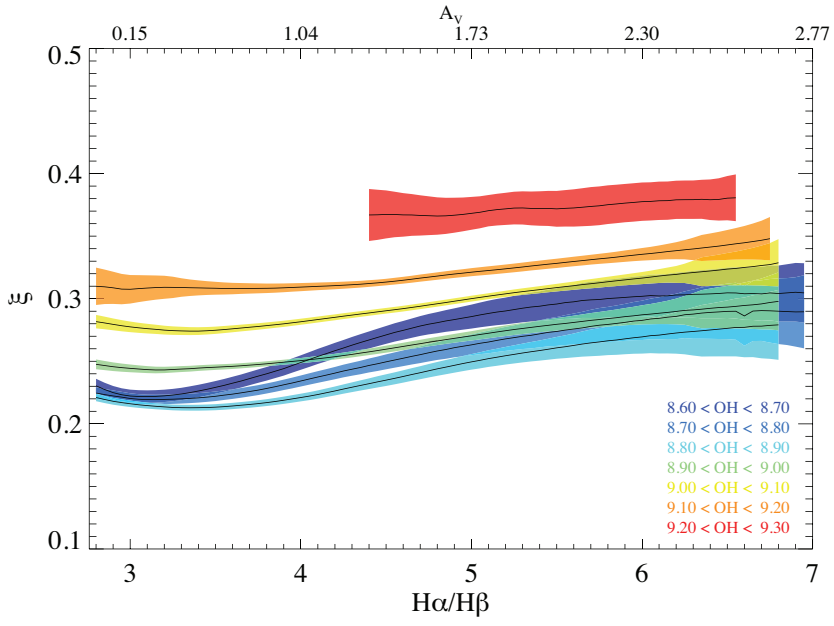

Figure 9. The dust-to-metal ratio of the gas as a function of the Balmer decrement in seven bins in oxygen abundance; the inferred $A_{V}$ using a Seaton (1979) extinction curve is shown on the top axis for reference. The shading indicate the uncertainty on the mean - the spread around the mean is considerably larger. It is clear that the trend with dust attenuation is similar at different metallicities although there is a trend for an increasing depletion at higher metallicity.

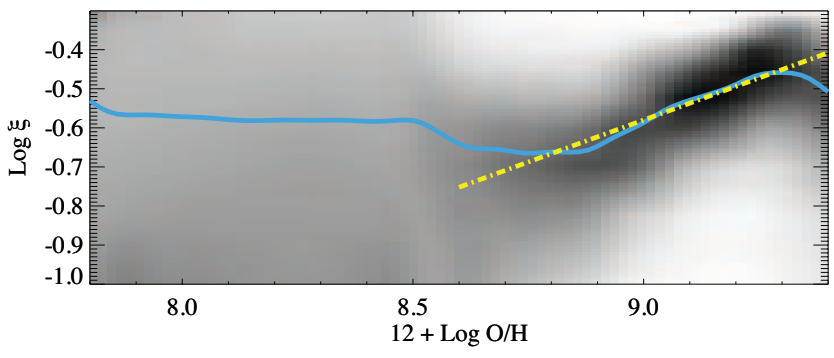

Figure 10. The dust-to-metal ratio of the gas as a function of the oxygen abundance. The grey scale shows the conditional likelihood of $\log \xi$ in bins of $\mathrm{O} / \mathrm{H}$. The solid blue line shows the median trend and the yellow dashed-dotted line shows the linear fit at high metallicity given in equation 27 Note the lack of constraints on $\xi$ below an oxygen abundance of 8.6.

\subsection{The dust-to-metal ratio as a function of metallicity}

We discussed the properties of $\xi$ in section 3 but we did not discuss what values our fitting procedure provides. In the Milky Way we know there is a correlation between depletion strength and (local) density (e.g. Jenkins 2009) and Fig. 9 shows that we find a similar result for our fits to star-forming galaxies in SDSS DR7. This figure plots our $\xi$ values against $\mathrm{H} \alpha / \mathrm{H} \beta$ as a proxy for line-of-sight attenuation. We indicate the V-band attenuation, $A_{V}$, obtained using a Seaton (1979) extinction curve on the top axis for reference. The figure was constructed by co-adding the individual PDFs of $\xi$ and $\mathrm{H} \alpha / \mathrm{H} \beta$. The PDF of the latter was calculated on a grid with bins of 0.043 while the PDF for $\xi$ was interpolated onto a grid with bin size 0.004 . We calculate the mean and uncertainty on the mean on the resulting combined twodimensional PDF and only show the trends where at least 10 galaxies contribute to the bin in $\mathrm{H} \alpha / \mathrm{H} \beta$. In this and the following figure we use all SF class galaxies in the SDSS since we are comparing two quantities derived within the same region, the figure does not change significantly except in the metallicity ranged spanned when limiting attention to a specific redshift range.

The solid line shows the mean and the shading the uncertainty on the mean for each trend. The results are shown for seven bins in oxygen abundance, from $0.5 Z_{\odot}$ to $\sim 3 Z_{\odot}$. We do not include lower abundance bins because, as we discuss next, we are unable to constrain $\xi$ accurately at low metallicity.

The first point to notice is that we do see stronger depletion in more dusty systems, similar to the trend seen along different sight-lines in the Milky Way although the significant difference in methodology bars us from making a much stronger statement, and the trend is fairly weak. The second point to note is that although the depletion appears to vary systematically with oxygen abundance, the dependence on attenuation appears to be the same for most metallicities.

This indicates a significant dependence of depletion on metal abundance and we make this explicit in Fig. 10] which shows the trend of $\xi$ with oxygen abundance for star-forming galaxies from the SDSS DR7. This uses the full likelihood distributions but is shown conditional on the oxygen abundance, with the solid line indicating the median to guide the eye. This shows two noticeable features. Firstly we can see a clear correlation between $\xi$ and $12+\log \mathrm{O} / \mathrm{H}$ at high metallicity - in the sense that we predict a stronger depletion of elements in galaxies with higher metallicity. For reference, the behaviour at high metallicity is well described by

$\log \xi=-4.45+0.43 \mathrm{OH}$

where $\mathrm{OH}=12+\log \mathrm{O} / \mathrm{H}$. This is a good fit to the data for $12+\log \mathrm{O} / \mathrm{H}>8.7$ and is overplotted as a dashed-dotted yellow line in the figure. But note that this includes the trend with attenuation as well thus the intrinsic trend at fixed attenuation is slightly shallower but we ignore this here. 


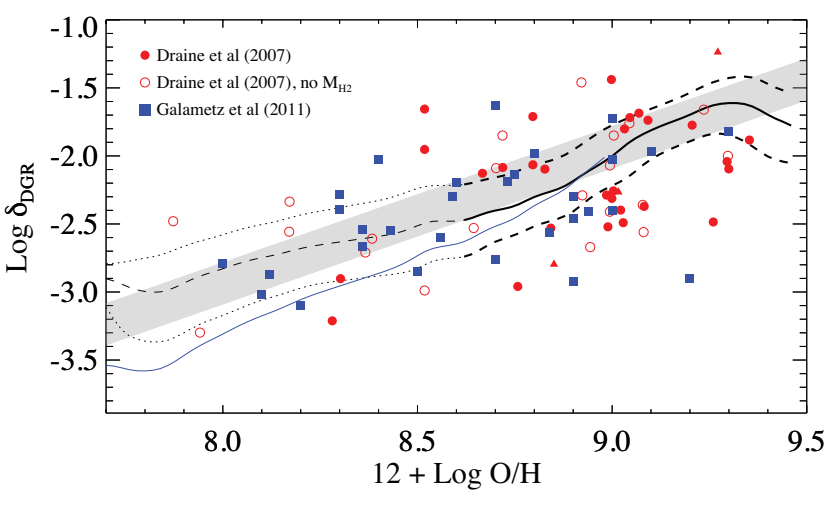

Figure 11. The solid/dashed line shows the median dust-to-gas ratio for the SDSS DR7 as a function of gas-phase oxygen abundance, with the dashed/dotted lines indicating the 68 per cent spread around this. The change in dashing at $12+\log \mathrm{O} / \mathrm{H}=8.6$ indicates the region where constraints on $\xi$ are poor. To illustrate the effect this has, the blue solid line shows the effect of assuming that $\xi$ follows equation (27). The open and filled circles show the $\delta_{\text {DGR }}$ estimates from Draine et al (2007) for SINGS galaxies - filled circles for galaxies with $\mathrm{CO}$ detections, empty circles for galaxies without this. The solid blue squares are from the study of nearby galaxies by Galametz et al. (2011). The grey shaded region shows the expected locus if the dust-to-gas ratio is scaled linearly with metallicity from the Milky Way value inferred by Jenkins (2009).

The second point to note in this figure, is that we cannot place any constraints on $\xi$ at metallicities lower than $12+\log \mathrm{O} / \mathrm{H} \approx 8.6$, or $\sim 50$ per cent solar, recall that the solar oxygen abundance for the CL01 models is 8.81 . This appears to be a consequence of the higher electron temperature in low metallicity gas and subsequent lower sensitivity to $\xi$ as indicated by Fig. 1. Thus we are unable to say whether the dust-to-metal ratio continues to decrease, as an extrapolation using equation (27) would predict, or whether the relationship at low metallicity is flatter, as for instance the chemical evolution models by Calura et al. (2008) suggest and which the fit results hint at.

In the following section we will therefore refer to two different estimates of $\xi$. Our first estimate will be that derived from the fitting method and hence which shows a flattening at low metallicity, the second will use the fit in equation (27) to adjust $\xi$ after the fact — we can do this because the model fits at low metallicity are approximately independent of $\xi$. In the rest of the paper we will for simplicity focus on the values provided by the fitting method with and without a prior on $\xi$.

\subsection{Dust-to-gas ratios as a function of metallicity}

The dust-to-gas ratio in galaxies is a crucial parameter for a wide range of models, and has been extensively modelled with focus on dust emissivity (see e.g. the compilation in Galametz et al. 2011). As remarked above a key ingredient in our approach to estimate gas content is that we can place constraints on the dust-to-metal, and by extension the dustto-gas, ratio

$\delta_{\mathrm{DGR}}=\xi Z$, using our emission line modelling. In this equation $\delta_{\mathrm{DGR}}$ is the ratio of dust mass density to that of the total gas mass. For comparison to other work, which focuses on the mass ratio of dust relative to hydrogen, we adjust our estimates here by a factor of 1.38 to take out the contribution of helium to the gas mass density, a factor which uses the solar abundance ratios from Asplund et al. (2009).

To test our estimates, we focus our attention on the $\delta_{\text {DGR }}$ versus oxygen abundance relation. We use the full likelihoods of these parameters and show the resulting distribution in Fig. 11] The solid/dashed black line shows the median $\delta_{\mathrm{DGR}}$ at the given oxygen abundance, while the dashed/dotted lines show the 68 per cent spread around the median. In section 5.1 above, we showed that at $12+$ $\log \mathrm{O} / \mathrm{H}<8.6$, our method is not able to place strong constraints on $\xi$ and as a consequence our estimate of $\delta_{\mathrm{DGR}}$ is much less certain. This region is therefore indicated with a different linestyle in the figure. To indicate the effect of modifying $\xi$, we overplot the result of modifying our $\delta_{\mathrm{DGR}}$ estimates using equation (27) as a thin blue line. At low metallicity the effect can be almost 0.5 dex.

We compare our estimates to those of Draine et al. (2007), who modelled the dust emission of SINGS galaxies using a range of MIR data, and those of Galametz et al. (2011) who used a similar approach but a different modelling technique. These are overplotted in Fig. 11 as red circles (open for galaxies lacking a CO detection) and filled squares respectively. For the Draine et al data we have calculated oxygen abundances using our models using the data described in Moustakas et al. (2010), kindly provided by J. Moustakas. For the Galametz et al data we use the metallicities provided in their table 1. For consistency with Galametz et al, we have adopted the $X_{\mathrm{CO}}$ dependency in equation (23) for all data shown here. For the Draine et al sample we use the gas masses measured within the region where dust emission is detected - see the discussion in Draine et al. (2007) for why this is the reasonable choice.

It is also interesting to compare our results with the simple assumption that $\delta_{\mathrm{DGR}}$ scales linearly with metallicity (c.f. Draine et al. 2007). To calculate this we have made use of the depletion patterns derived by Jenkins (2009), adjusted to the CL01 abundances. This leads to a prediction for $\delta_{\mathrm{DGR}}$ of

$\delta_{\mathrm{DGR}}=\left(5.3 \times 10^{-3}-1.1 \times 10^{-2}\right)\left(\frac{\mathrm{O} / \mathrm{H}}{(\mathrm{O} / \mathrm{H})_{\odot}}\right)$.

Where the range is given for $F_{*}=0$ to $F_{*}=1$. The resulting region is shaded in grey in Fig. 11 It is clear that the linear scaling appears to be a decent overall description but our data differ from this somewhat in detail, having a steeper slope down to $1 / 2 Z_{\odot}$, and if we extrapolate with equation (27) we differ rather more at low metallicity.

The main conclusion from this test is that there is a good general agreement between the measurements in the literature and our values despite the very different techniques used. A notable difference between our method and the other efforts in the literature is that our measure of $\delta_{\mathrm{DGR}}$ is a local measure in contrast to methods that use data obtained over different areas and with different spatial resolution.

It is also worth asking whether a scaled dust-to-gas ratio would be sufficient, since that appears to capture much of 
trend in Fig. 11 This does of course depend on the question asked, but it does rely on $\xi$ being approximately constant and Fig. 9 and 10 show that this is a questionable assumption in detail. Ignoring the variation in $\xi$ would lead to systematic errors in the dust-to-gas ratio that could amount to as much as a factor of two across the bulk of the galaxies in the SDSS and this would be a significant source of error in many studies.

\subsection{Comparisons to resolved gas masses}

We can carry out a direct test of our gas measurements when we have spectroscopic observations of galaxies where we also have interferometric maps in $\mathrm{HI}$ and $\mathrm{CO}$. We will here use two sources of such spectra: fibre spectra from the SDSS and spectra from the IFU mosaic of NGC 628 by Sánchez et al. (2011). We show these using different colours below to verify that the method works equally well for both classes of spectra.

As an illustration of our resolved maps, Fig. 12 shows the $r$-band, $\mathrm{HI}$, and CO images of M101 (NGC 5457) with the location of spectra from SDSS indicated as red squares and NGC 628 was shown earlier in Fig. 8. The $r$-band image is a mosaic of SDSS $r$-band images, the H I map is from the THINGS survey and the CO map from the HERACLES survey. We have explored apertures from 6" to 24" when measuring $\Sigma_{\mathrm{HI}}$ and $\Sigma_{\mathrm{CO}}$, corresponding to $0.2-0.8 \mathrm{kpc}$ at the adopted distance for M101 of 7.4 Mpc (Karachentsev et al. 2004), and comparable sizes for the rest of the sample. Here we will only show results for apertures of 12 " since this is close to the angular resolution of the HERACLES data (Lerov et al. 2009b). Note that this aperture is four times that of the SDSS data, for which all $\Sigma_{\text {gas }}$ measurements are obtained in an aperture of 3". For the NGC 628 data we can synthesise spectra in arbitrary aperture sizes but since we work with the emission line maps rather than the data cube, this is not an exact sum of the emission flux within the aperture.

For each spectrum we run our model fits as detailed above and inspect each resulting fit in detail. The SDSS spectra do not sample [O II] 3727 and generally have fairly low $\mathrm{S} / \mathrm{N}$ which occasionally leads to poorly constrained fits, while for the NGC 628 mosaic, the [S II] line fluxes are occasionally significantly off and we therefore do not include [S II] in the fits shown here. We exclude fits that give multi-modal PDFs and very low S/N spectra - quantitatively we make a cut at a Kullback-Leibner divergence of 15 for the $\Sigma_{\text {gas }}$ PDF. In practice this corresponds to spectra with such weak emission lines that no constraint can be had for $\Sigma_{\text {gas }}$. This results in a total of 104 (59 from SDSS, 45 from NGC 628) high-quality measurements. The results do not depend much on these cuts because these poor fits merely add scatter to the relation and since the uncertainty is large their effect on any fit to the data is minor and we have verified that including them does not significantly affect the conclusions below.

We compare the $\Sigma_{\text {gas }}$ from our models with the values extracted from the $\mathrm{H}$ I+CO maps in Fig. 13. The left panel plots $\Sigma_{\mathrm{HI}}$ for these regions against the spectroscopic $\Sigma_{\text {gas }}$ estimates. The point sizes in this figure are inversely proportional to the uncertainty estimate on $\log \Sigma_{\text {gas }}$ between 0.1 and 0.6 dex. The blue line shows an unweighted bisector fit to the data which has a slope of 0.79 and an intercept of 0.43 . The dashed red line shows the 1-1 relation with a factor of 2 offset indicated by the dotted lines. While the data clearly correlate with each other, the slope is noticeably different from 1 .

The right panel shows the same but now using the total gas mass on the abscissa. We calculated the molecular mass using the metallicity dependent $X_{\mathrm{CO}}$ from equation (23) with the metallicity from our model fit. We have also applied the broad prior on $\xi$ although the effect of both of these corrections are moderate as the majority of the spectra are close to solar metallicity. For NGC 4449 (red filled circle) we use the observations of Hunter et al. (2000), whose region B overlaps with several of our spectra and when adjusted to our default $X_{\mathrm{CO}}$ has $M_{\mathrm{H}_{2}} / M_{\mathrm{H}_{\mathrm{I}}}=0.225$. The agreement is better in this panel with the bisector fit having a slope of 0.93 with an intercept of 0.03 .

The overall agreement must be said to be good as long as the total gas mass is used, showing explicitly that the method traces the total gas surface mass density. The slope and intercept does depend somewhat on the aperture used for the gas measurements, whether a metallicity dependent $X_{\mathrm{CO}}$ is used and whether or not a $\xi$ prior is applied. For apertures of either 12 " or 24 ", the slope of the best unweighted bisector fit varies from 1.06 to 0.93 and the intercept from -0.15 to 0.06 , thus fully consistent with a $1-1$ relation.

The scatter around the best linear fit varies from 0.30 to $0.36 \mathrm{dex}$, which is mostly explained by the uncertainties on the variables. It is difficult to accurately quantify the intrinsic scatter in this relation as it depends sensitively on the uncertainty estimates on the $\mathrm{CO}$ measurements which are hard to quantify due to the aperture mismatch between the SDSS and the CO data, as well as uncertainties in the $\mathrm{CO}-\mathrm{H}_{2}$ conversion factor, but fitting a linear relation with intrinsic scatter following Kelly (2007) the data are consistent with a linear relation with intrinsic scatter of $0.1-0.15$ dex.

Thus we can conclude that at least over the range of gas surface densities from $10-100 \mathrm{M}_{\odot} \mathrm{pc}^{-2}$, our spectroscopic method gives results in agreement with those inferred from $\mathrm{H} \mathrm{I}+\mathrm{H}_{2}$ maps to within a factor of two. Although shown only for an aperture of 12", this conclusion is robust to the aperture size chosen. Given the lack of suitable comparison data, we are unable to extend this to lower densities but when applied to SDSS data the method appears well behaved down to at least a surface gas mass density of $\sim 2 \mathrm{M}_{\odot} \mathrm{pc}^{-2}$ where our sample starts to peter out.

The figure also shows that our estimator provides a measure of the total gas mass. As an example to highlight this, the nuclear spectrum in M101 (1323-52797-012) corresponds to a location with essentially no $\mathrm{HI}$ gas and the resulting mass density is plotted as an open circle in the right panel of Fig. 13 and connected to the total $\left(\mathrm{H} \mathrm{I}+\mathrm{H}_{2}\right)$ mass density by a green line with an arrow.

There are however some cases where the method fails. The three main outliers are indicated with open squares in the figure. From left to right these are: the nuclear bar in NGC 2903 (2289-53708-615), which is well-known to have a particularly high molecular gas density (e.g. Leroy et al. 2009b), an off-centre region in NGC 3627, and a nuclear spectrum in NGC 3351. The off-centre region in NGC 3627 has strongly discrepant $\mathrm{H}_{2}$ estimates between BIMA and 

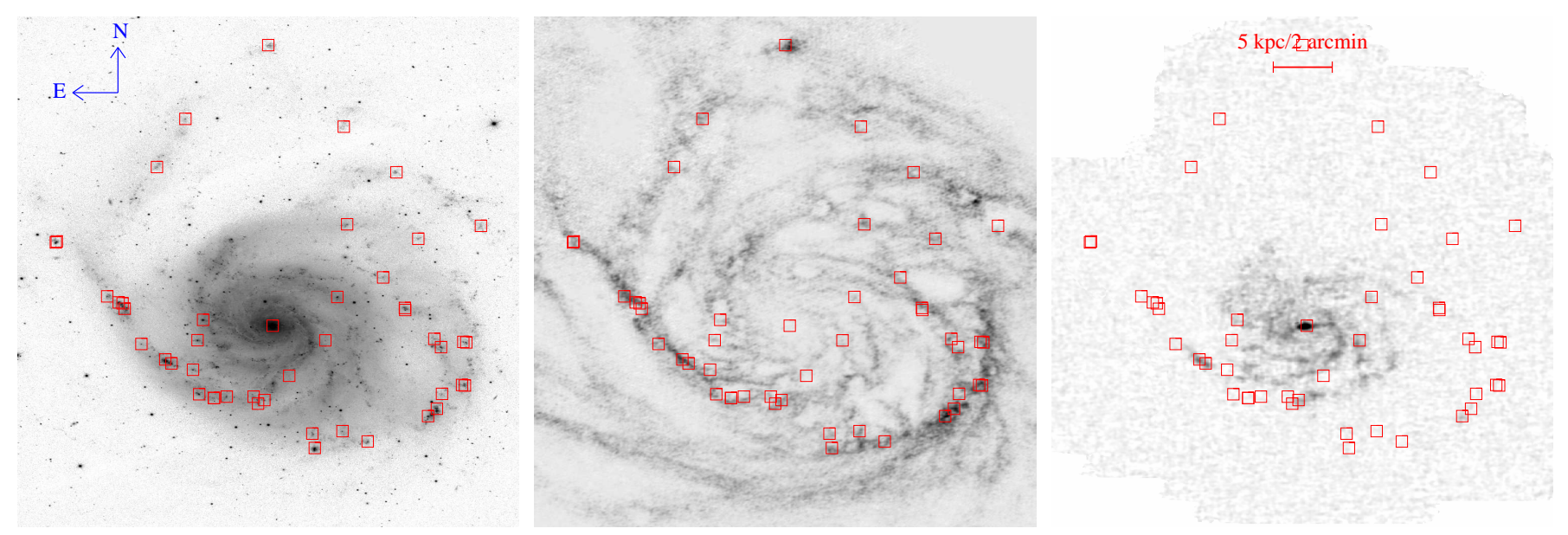

Figure 12. Left: SDSS r-band image of M101 with the location of SDSS spectra overlaid as red boxes. Middle: H I map of M101 from the THINGS survey. Right: The CO gas mass map of M101 from the HERACLES survey.

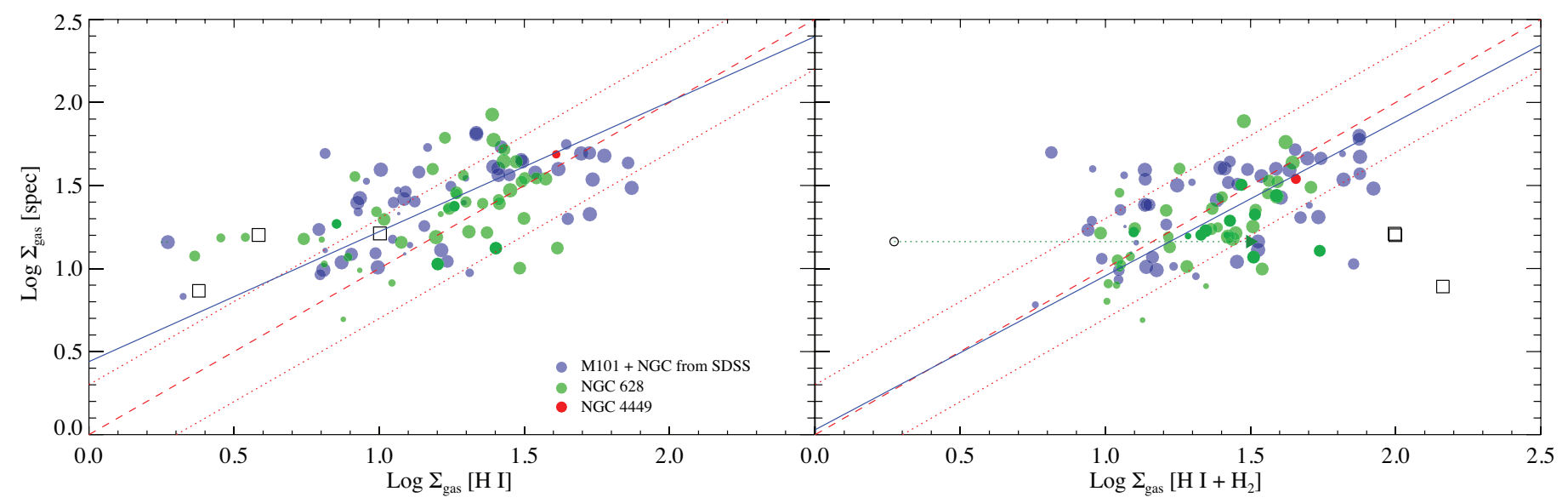

Figure 13. Left: Surface-mass density of H I measured in a 12" aperture versus our spectroscopic gas estimates for 59 locations in 11 nearby galaxies with SDSS spectroscopy (blue) and 45 locations in NGC 628 (green). The red solid symbol is for NGC 4449 . The points have been scaled according to the uncertainty estimate so that the largest circles correspond to objects with $1 \sigma$ uncertainty on log $\Sigma_{\text {gas }}$ less than 0.1 dex. The smallest symbols correspond to uncertainties larger than 0.6 dex. The dashed red line shows the $1-1$ relation, with the dotted lines either side showing a factor of 2 spread. The solid blue line shows the best-fit bisector fit to the data. Right: Like the right-panel but now plotting the total gas content on the x-axis. We show this for a metallicity dependent $X_{\mathrm{CO}}$ and using the broad $\xi$ prior. The green line with an arrow shows the contribution of $\mathrm{H}_{2}$ to the central aperture in M101 with the location for $\mathrm{H}$ I only shown as an open circle. From left to right the open squares indicate the position of NGC2903-00, NGC3627-01, and, NGC3351-00 see the text for a discussion.

HERACLES and if BIMA were used instead of HERACLES it would fall close to the 1-1 line, but the nuclear spectra have consistently high mass estimates from BIMA and HERACLES and the disagreement with the spectroscopic estimates appears to be real. Quantitatively we start to see a systematic disagreement between our spectroscopic gas estimator and the $\mathrm{CO}+\mathrm{H}$ I maps at surface mass densities of gas above $\sim 75 \mathrm{M}_{\odot} \mathrm{pc}^{-2}$.

It is important to test whether the agreement between the two gas mass measurements depend on other properties of the regions sampled in a systematic manner. We therefore calculate the difference between $\log \Sigma_{\text {gas }}$ inferred from $\mathrm{HI}+\mathrm{CO}$ data, the "direct" measure, and the spectroscopic estimate. In Fig. 14 we plot this difference as a function of four observables. On the left we show it as a function of
$\Sigma_{\mathrm{HI}}$ and we see no clear evidence of a systematic mismatch between the two as a function of $\mathrm{H}$ I surface mass density.

The second panel shows the difference as a function of equivalent width of $\mathrm{H} \alpha$. No significant trend is seen, although we note that the systems with high total gas mass density tend to have low EWs. This is due to a combination of these being nuclear spectra, where the background stellar light is significant, and to the star formation here typically being obscured. There are no NGC 628 symbols here as the data release does not provide equivalent widths.

In the third panel we plot the residuals against local colour (fibre magnitudes for SDSS and aperture photometry on the SDSS images of NGC 628 otherwise). Here we see a slight systematic offset at the red end, which is due to the nuclear spectra, where the very high $\mathrm{H}_{2}$ densities are found. This could conceivably be used to identify possible cases 


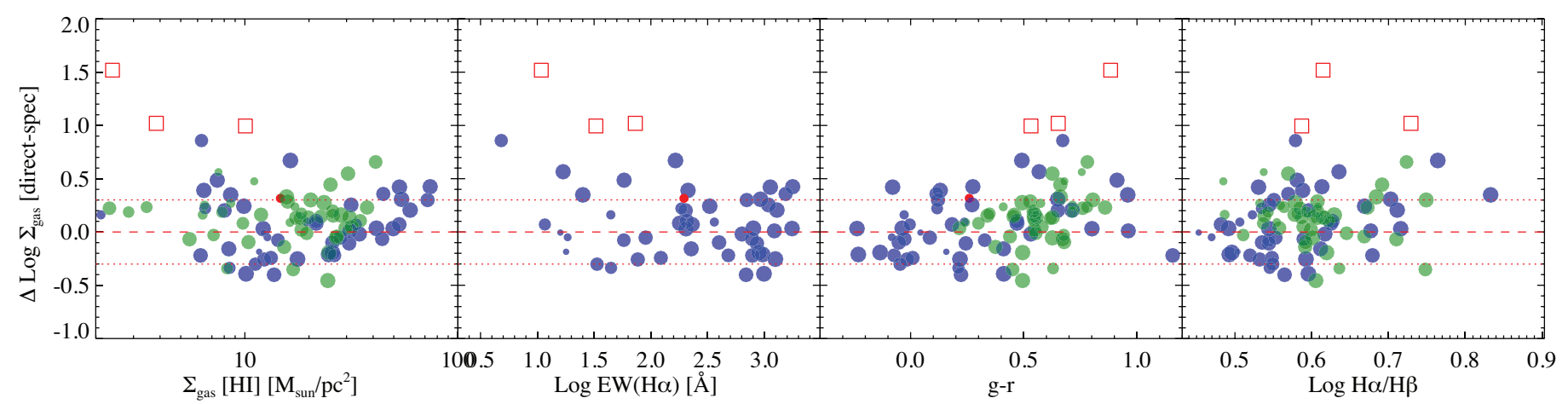

Figure 14. The difference between the spectroscopic and direct gas mass estimates in 12" apertures plotted against the H i surface mass density (left panel), equivalent width of $\mathrm{H} \alpha$ (second panel), the local $g-r$ colour (third panel) and $\mathrm{H} \alpha / \mathrm{H} \beta$ flux ratio (rightmost panel). The SDSS data are shown in blue and the NGC 628 data in green, the other symbols are as in Fig. 13 The red horizontal line shows the zero level and the dashed lines around this shows a factor of 2 of scatter. We do not have equivalent width measurements for H $\alpha$ for NGC 628.

where our method fails, but we do not attempt to do that here.

The final panel shows the residuals as a function of the Balmer decrement. Here we see no trend showing that our method works well for a wide range of optical depths. We have further verified that no systematic trends are visible as a function of oxygen abundance or the signal-to-noise of the spectroscopic gas estimate.

We conclude from this that the method appears to work well for surface densities of gas $<50 \mathrm{M}_{\odot} \mathrm{pc}^{-2}$ but appears to break down at surface densities $>100 \mathrm{M}_{\odot} \mathrm{pc}^{-2}$. This limitation is expected, because at high optical depth the emission lines we see will typically come from a skin outside the high optical depth region.

While this is a real limitation, we should emphasise that it is a problem only for nuclear spectra. The scale sampled by a 3" fibre varies from 42 pc (NGC 4214) to 200 pc (NGC 3198 ), while the median fibre in the SDSS $(z<0.2)$ subtends $\sim 5 \mathrm{kpc}$. On larger scales we will not be dominated by these extreme conditions in the majority of galaxies.

\subsection{Moving from smaller to larger scales}

The preceding section showed that our method provides gas mass measurements in good agreement with resolved gas measures for all but the highest gas densities. Formally, however, our derivation in section 2 is only valid for angleaveraged quantities, thus we would like to carry out our checks for larger samples and since most of the SDSS fibre spectra sample much larger scales than the $\sim 100 \mathrm{pc}$ our tests above explored, we would also like to extend our tests to larger spatial scales.

In lieu of large samples of more distant galaxies with H I maps, we have two options. We start by synthesising apertures of increasing size using the IFU mosaic of NGC 628. This allows us to test how well we can recover the $\mathrm{H} \mathrm{I}$ content within the optical radius of the galaxy. The result of this exercise is shown in Fig. 15] The box plots summarise the PDFs from our fits, while the blue line shows the total gas mass estimated from the $\mathrm{H}_{\mathrm{I}}$ and $\mathrm{H}_{2}$ maps using the metallicity dependent $X_{\mathrm{CO}}$ in equation (23). The red line shows the result for a constant $X_{\mathrm{CO}}$.

The overall agreement is very good as long as we take

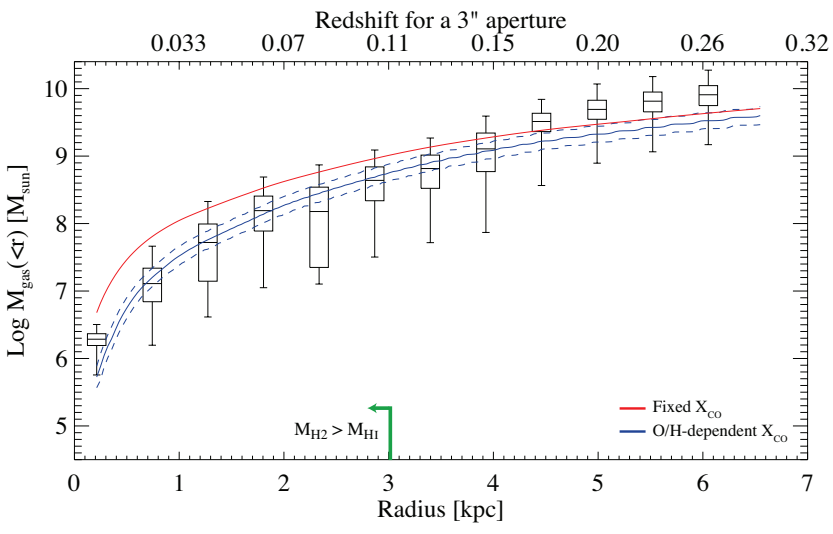

Figure 15. The total gas mass within apertures with different radii for NGC 628 . The results are plotted as box-plots with the box enclosing 50 per cent around the median of the PDF, and the error bars enclosing 95 per cent of the likelihood. The solid red line shows the total mass estimate from the $\mathrm{H}$ I and $\mathrm{CO}$ maps assuming our reference $X_{\mathrm{CO}}$. The blue line with dashed error lines shows the result of assuming a metallicity dependent $X_{\mathrm{CO}}$, using the full PDF of $12+\log \mathrm{O} / \mathrm{H}$ from our fits. The flux uncertainties for each radius were scaled to have the same $\mathrm{S} / \mathrm{N}$ as the second radial bin before running the fits. The top axis shows the redshift at which a 3" SDSS fibre would enclose a physical aperture with radius given on the lower axis. Note that as this is a cumulative plot, the error bars are not independent.

into account the metallicity dependence of $X_{\mathrm{CO}}$, with the main uncertainty coming on the largest scales. The agreement is typically better than a factor of 2 across a wide range of sampled sizes. It is also worth noting that the agreement appears to be equally good regardless of whether the gas is dominated by $\mathrm{H}_{2}$ (within a radius of $3 \mathrm{kpc}$ ), or atomic gas. There does seem to be a tendency for systematic offsets at very large radii. The reason for this appears to be that only regions of relatively high star formation activity have sufficient $\mathrm{S} / \mathrm{N}$ to have their line fluxes measured in the IFU data. These regions are typically more gas rich than the average at those radii and hence the spectroscopic estimates are biased high, but this should not be taken to be a failure of the method, rather it is a sign that we are not comparing like with like here. 
We can extend this kind of test to the full SDSS sample by exploiting the fact that the SDSS fibre aperture spans different physical sizes at different redshifts, thus as redshift increases we should trace a larger and larger fraction of the gas content of the galaxy. This will not provide total gas masses for the galaxies, but at $z \sim 0.2$ the SDSS fibre will subtend $\sim 10 \mathrm{kpc}$ and encompass $\approx 50$ per cent of the light of the galaxy in our sample, so one might expect that the gas content is more representative for the total.

To test this we wish to compare our $r_{\text {gas }}-M_{*}$ relation against the $M_{\mathrm{HI}} / M_{*}-M_{*}$ relation. This has been well characterised locally Bothun 1984; Kannappan 2004; Catinella et al. 2010, 2012), and within large enough apertures, the dominant gas phase might expected to be atomic (but see Saintonge et al. 2011b). As an example, ignoring $\mathrm{H}_{2}$ for NGC 628 underestimates the total gas mass by $<0.2$ dex as long as we sum in apertures with radius $>4.5 \mathrm{kpc}$. Thus the $M_{\mathrm{H}} / M_{*}-M_{*}$ relation should be a good proxy for the $r_{\text {gas }}-M_{*}$ relation as long as the $\mathrm{H}$ I determinations are from a region larger than this.

The result of this comparison is shown in Fig. 16. The solid black line here shows the $r_{\text {gas }}-M_{*}$ trend found for starforming galaxies in the SDSS using the fibre spectra. Each panel corresponds to a range in physical size subtended by the SDSS 3" fibre, as indicated in each panel. The blue and red points are from the ALFALFA and GASS H I surveys respectively, note that for GASS we only use the $\mathrm{HI}$ detections as the gas mass estimates using our method only includes objects with measured gas content. Since only H I detections are included these are likely to be biased high, so we also include the median $r_{\text {gas }}$ predicted using the photometric gas content technique introduced by Zhang et al. (2009, Z09), discussed further below, plotted as the solid purple line. This is calculated for all galaxies falling in the indicated radius/redshift bin.

When sampling small scales, the gas content is low relative to the stellar content, as one would expect in most optically bright galaxies (see for instance the atlas in Leroy et al. (2008)). Nevertheless when the 3" fibre samples regions with diameter in excess of $5 \mathrm{kpc}$, we approach the same scaling law found by the integrated $\mathrm{H}$ I measurements but we always fall below the locus of ALFALFA and GASS detections. This is not unreasonable both because $\mathrm{H}$ I-detected galaxies form a gas-rich subset of all galaxies, and because gas disks generally are more extended than the stellar disks of galaxies. We do, however, fall above the locus predicted by the photometric estimator at high mass, indicating that the sample for which we can estimate gas masses is a biased sample and we will return to this topic in section 6.3 below.

While this is an indirect test of our method, it is an important consistency check and taken together with the results for NGC 628 we argue that it is consistent with the assumption that our method can give reliable estimates of $\Sigma_{\text {gas }}$ within the optical disk of a galaxy. Taken together with the results of the previous section we conclude that the technique works well from $\sim 100$ pc scales up to $\sim 10 \mathrm{kpc}$ scales. Our method will, however, not work outside the optical disk of a galaxy because it relies on a background source that the attenuation can be measured against.

We have also verified, but do not show, that we can recover the scaling law between stellar surface mass den- sity and the star formation efficiency (SFR/M $\left.M_{\text {gas }}\right)$ found by Lerov et al. (2008) using our method.

In theory we could make the preceding comparison much more direct by exploiting the large samples of galaxies with integrated H I masses. In practice, however, a direct comparison is problematic because of the very large difference in apertures between the 3" aperture of the SDSS spectrum and the 3.5' Arecibo beam. We have carried out this test and the details are discussed in Appendix $\mathrm{A}$ but although the results are encouraging, and consistent with the conclusions of the preceding tests, the strength of this test is limited by the significant systematic uncertainties on the aperture corrections. One might wonder if we could improve on this by focusing on a comparison for the more distant objects where the SDSS fibre subtends a considerably larger spatial size. However at those redshifts we hardly have any H I data to compare to so at present this is not a practical approach to take.

\subsection{Comparison to other techniques}

In closing this section, it is useful to compare our method to a selection of indirect gas predictors in the literature. These are generally calibrated on a sample of nearby galaxies and applied to much larger samples. Early work on this made use of the fact that there is a good correlation between galaxy colour and the ratio of H I mass to B-band luminosity (Roberts 1969; Bothun 1984). More recently, Kannappan (2004, K04) found a tight relationship between galaxy colour, k-corrected to $z=0$, and $r_{\mathrm{HI}}=M_{\mathrm{H} \text { I }} / M_{*}$ :

$\log r_{\mathrm{HI}}=1.46-1.06(u-r)$,

and a somewhat tighter relation with $u-K$ which we will not use here. She showed that the scatter in this relation was only 0.42 dex and could therefore be used to give reasonable estimates of a galaxy's gas content on the basis of its colour.

An improved version of this was presented by Z09 who showed that an even tighter relationship could be had by including the $i$-band surface brightness, $\mu_{i}$ :

$$
\log r_{\mathrm{HI}}=-1.73238(g-r)+0.215182 \mu_{i}-4.08451,
$$

with a scatter of only 0.31 dex. A similar scatter was also found for massive galaxies based on the GASS survey by Catinella et al. (2010), incorporating instead the near-UV to $r$ colour and stellar mass surface density, $\mu_{*}$. For simplicity we will use the Z09 relationship in equation (31), as this will suffice for our needs here.

We calculated these gas mass estimates for the SDSS and NGC 628, when necessary calculating kcorrected colours using version 4.1 .4 of the kcorrect code (Blanton \& Roweis 2007). In contrast to Z09 we also use the stellar mass estimates from the kcorrect code. For NGC 628 we obtained photometry in the 6", 12" and 24" apertures on the basis of SDSS imaging of the object.

An alternative technique, widely used at high redshift (e.g. Erb et al. 2006), is to invert the Kennicutt-Schmidt (K-S) scaling law (Schmidt 1959; Kennicutt 1998) between the surface mass density of gas and star formation rate per unit area:

$\Sigma_{\mathrm{SFR}}=2.5 \times 10^{-4} \Sigma_{\text {gas }}^{1.4}$ 


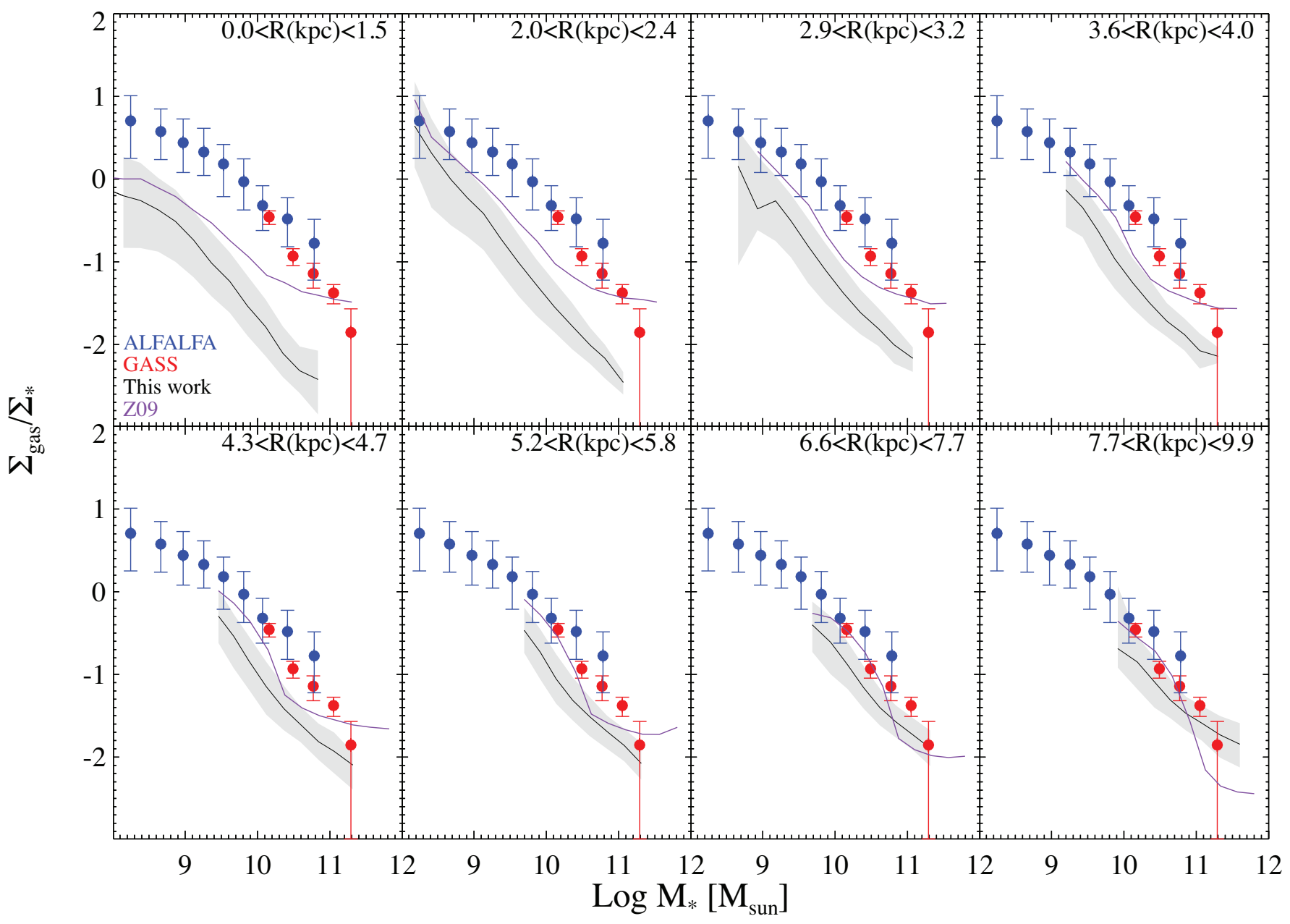

Figure 16. Each panel compares the scaling law between stellar mass and $r_{\text {gas }}$ in for star forming galaxies in the SDSS (solid line with $1 \sigma$ scatter shown as shaded grey region) to the scaling law between $M_{\mathrm{H}_{\mathrm{I}}}$ and stellar mass found using the ALFALFA survey (blue points), the GASS survey (Catinella et al. 2010) (red points) and the $r_{\text {gas }}$ values predicted using the Z09 photometric gas content estimator (purple solid line). The different panels correspond to different redshifts, here indicated by the size subtended by the 3" fibre used by the SDSS spectrograph. At the lowest redshifts the gas fractions are clearly below the mean gas fractions from the H I surveys because we sample the central parts of galaxies which are known to be less gas dominated, but as we sample more than $\sim 5$ kpc the trends found approach each other.

where $\Sigma_{\mathrm{SFR}}$ is in units of $\mathrm{M}_{\odot} \mathrm{yr}^{-1} \mathrm{kpc}^{-2}$ and $\Sigma_{\text {gas }}$ is in units of $\mathrm{M}_{\odot} \mathrm{pc}^{-2}$, and where uncertainties on the parameters are suppressed. We have written the equation here for a Salpeter IMF. To follow common usage in the literature we derive the SFR from the $\mathrm{H} \alpha$-luminosity and correcting for dust attenuation using $\mathrm{H} \alpha / \mathrm{H} \beta$, assuming a fixed Case B value of 2.86 and $\tau(\lambda) \propto \lambda^{-1.3}$, but we have checked that we get similar results if we use the more sophisticated modelling of B04.

Fig. 17 shows the comparison of these three gas estimators together with our spectroscopic method against the total gas content for the regions in nearby galaxies and NGC 628. We have excluded the three regions marked with a red square in Fig. 13 for clarity as these are outliers for all the estimators. The dashed line in each panel is a 1-1 relation, while the dotted line is the best unweighted bisector fit to the data. For the $\mathrm{K}-\mathrm{S}$ scaling relation and our method we also indicate the median perpendicular distance to the 1-1 line, $m_{p}$, as well as the perpendicular scatter around this line, $\sigma_{p}$.

We can immediately see that the K04 and Z09 estimators correlate poorly with the local gas content. This is not unexpected because they were calibrated on the integrated gas content of galaxies and they should not be expected to work for resolved properties. While a slightly unfair comparison, it does highlight very clearly the problem of applying a relation calibrated on local data outside of its range of validity - a similar caution would be valid for an application at high redshift as well. For statistical studies of total gas content in low redshift galaxies, they are however well suited.

The Kennicutt-Schmidt relation, in contrast, is sensitive to local properties and it clearly does a much better job than the colour-based techniques in Fig. 17. Nevertheless, the scatter around the 1-1 line is 85 per cent larger then with our method and the correlation has a slope different from one. This is not surprising as it is known that the $\mathrm{K}$ - 

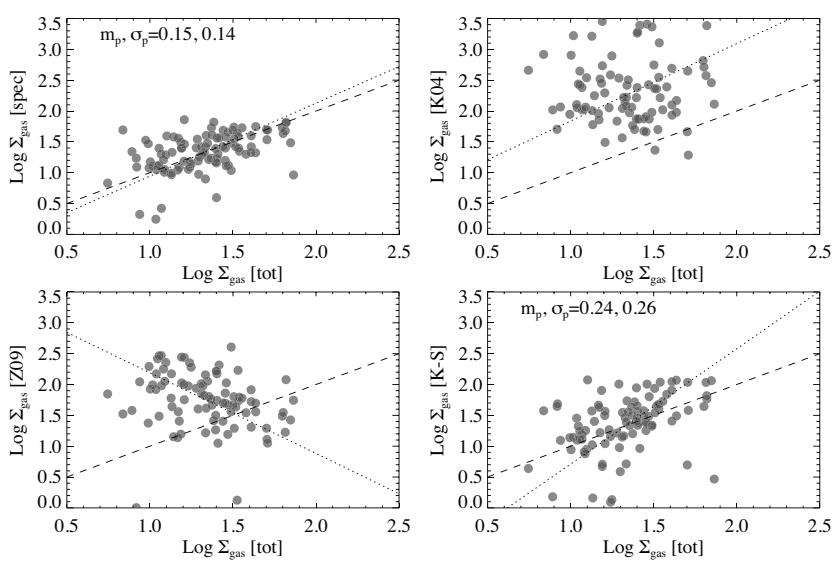

Figure 17. A comparison of four different gas mass estimators plotted against the total ( $\mathrm{H}$ I + molecular) gas mass using a metallicity dependent $X_{\mathrm{CO}}$ on the x-axis. The top left panel shows our spectroscopic gas mass estimates, the comparison in the top right panel uses the $r_{\mathrm{HI}}-(u-r)$ calibration from K04, the bottom left the calibration from Z09 and the bottom right shows the results of inverting the Kennicutt-Schmidt $(\mathrm{K}-\mathrm{S})$ relation from Kennicutt (1998). The dashed line is the 1-1 line, while the dotted line shows the best linear bisector fit to the data shown. Our estimator clearly does best, while the $\mathrm{K}-\mathrm{S}$ relation does well but has more scatter and a slope differing somewhat from 1.

$\mathrm{S}$ relation shows significant variations (e.g. Kennicutt et al. 2007; Bigiel et al. 2008). But the main problems with this method are not seen in this figure: a) It is calibrated on nearby galaxies so it is not a given that it would work for all galaxies and at all redshifts and b) it is a transformation of the star formation rate so it would be meaningless to plot it against a star formation rate indicator.

\section{THE CENTRAL GAS CONTENT OF LOCAL GALAXIES}

As an example application of the methodology outlined above we now turn to look at how the central gas content in star-forming galaxies in the local Universe varies with the physical properties of the galaxies. We will concentrate on galaxies from the SDSS DR7 for which we have fibre spectra.

Since the fibre spectra subtend a fixed size in observer space we are faced with the fact that we sample different parts of the galaxies at different redshifts. As discussed in appendix $\mathrm{A}$ aperture corrections suffer from substantial systematic uncertainties thus we prefer here to focus on galaxies with for which the aperture samples similar scales. Given the quantities we have available this could imply focussing either on a fixed physical scale or on a fixed fraction of the galaxy size. The former ensures that the galaxies considered all lie in a narrow redshift range, while the latter option mixes galaxies across a range of redshifts but might provide a more physically meaningful result.

To illustrate the variation in properties with these two different definitions of central regions as well as to explore the impact of different aperture sizes, we calculate $r_{\text {gas }}$ in bins of stellar mass as a function of aperture size in physical units, as well as in bins of the size of the aperture relative to the half-light radius of the galaxy. The results are shown in
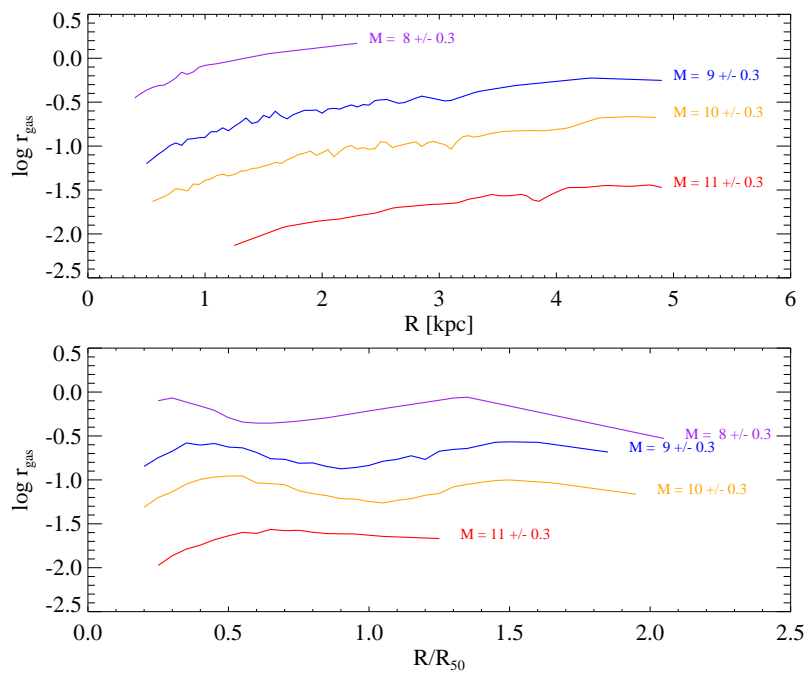

Figure 18. Top panel: The median $\log r_{\text {gas }}$ as function of radius for four ranges in stellar mass. Note that in each range we see a similar trend towards higher $r_{\text {gas }}$ when we sample larger radii but that the typical $r_{\text {gas }}$ increases with decreasing stellar mass. Bottom panel: The median $\log r_{\text {gas }}$ as a function of the size of the fibre in units of the half-light radius of the galaxy. Note the lack of correlation between the normalised radius and the gas ratio.

Fig. 18 and show that $r_{\text {gas }}$ is effectively independent of the aperture size in units of the half-light radius of the galaxy out to $\sim 2 \times R_{50}$. If we contrast this with Fig. A2 we see some differences which in part is due to that figure using $\mathrm{H} \mathrm{I}$ only, adding $\mathrm{H}_{2}$ will flatten the central profiles, but it is also likely that part of this flat structure is a sign of the biased nature of our sample relative to the total galaxy population and we will return to this below.

Since both these definitions of "central" are defendable, we will show results for both in the following. Specifically we will look at gas content in the central $2 \pm 0.5 \mathrm{kpc}$, corresponding to $0.05<z<0.09$, when we fix the physical scale. For simplicity we do not attempt to correct for aperture effects within this redshift interval, but based on Fig. 18 these would be \pm 0.1 dex in median. We also will show results when the sample is limited to $0.7<r_{n}<1.4$, with $r_{n} \equiv R / R_{50}$. The samples resulting from these two cuts both include $\sim 7 \times 10^{4}$ galaxies. These definitions should be considered a central, but not nuclear, gas content of starforming galaxies in the nearby Universe. The qualitative results in the following section are only weakly sensitive to this choice of aperture but the quantitative results do of course change. The ultimate consequence of this is that the results we derive below are not representative for the total gas content of galaxies, for which suitable studies are available in the literature (e.g. Catinella et al. 2012; Schiminovich et al. 2010; Saintonge et al. 2011a).

\subsection{The central gas-to-stellar ratio in galaxies in the nearby Universe}

We show our inferred variation in $r_{\text {gas }}$ in the central regions of galaxies in Fig. 19. The details of the construction of these maps are given in appendix $\mathrm{C}$ but they are basically 

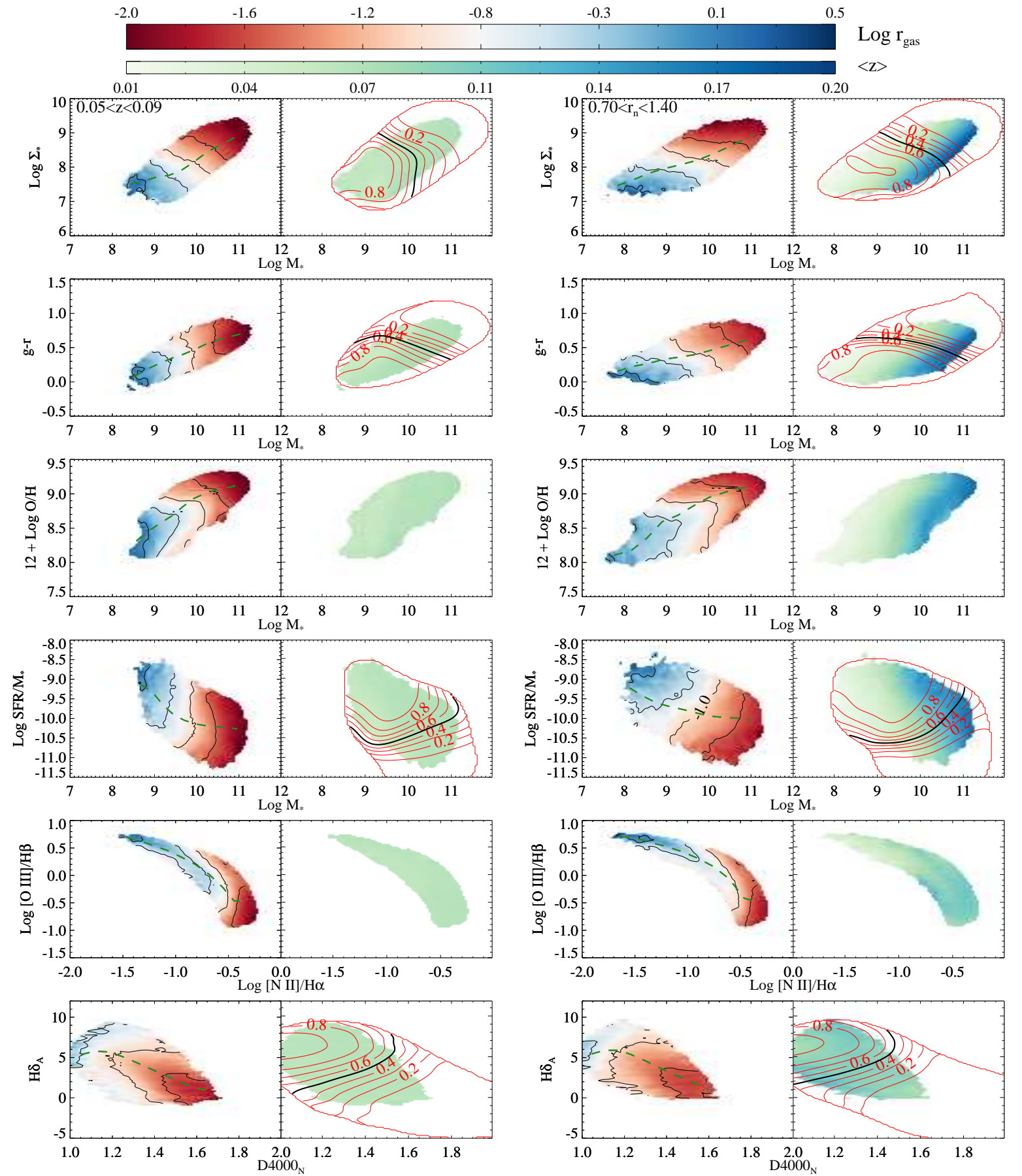

Figure 19. The gas content of star-forming galaxies within $2 \pm 0.5 \mathrm{kpc}$ (left column) or within $0.7<r_{n}<1.4$ (right column) in the SDSS as a function of various physical parameters. Each quantity is shown with the average $\log r_{\text {gas }}$ in the left panel with blue to red colour scale, while the panel of the right shows the mean redshift in the colour scale (green to blue) and overlaid a contour map of the fraction of all SDSS galaxies that we have gas measurements for (see text for details). The top row shows the trend with stellar mass and stellar surface mass density, while the following rows show $r_{\text {gas }}$ in the $\log M_{*}$ versus rest-frame $g-r$ colour plane, the log $M_{*}-$ oxygen abundance plane, the $\log M_{*}$ versus specific SFR plane, the BPT line ratio diagram and finally, the D $4000_{N}$ versus $\mathrm{H} \delta_{A}$ plane. Contour lines are overlaid in steps of $0.5 \mathrm{dex}$ from $\log r_{\text {gas }}=-2$. In the mean redshift panel, the completeness contours are not shown for the BPT plane or the $\log M_{*}$-oxygen abundance plane since essentially all galaxies that we can plot in these planes have gas mass estimates. Notice the very regular trend with various parameters but note in particular that the trends are not a function of stellar mass only, or indeed any other single parameter considered. The dashed green line in each panel shows the median value of the quantity plotted on the $\mathrm{y}$-axis as a function of the parameter on the $\mathrm{x}$-axis. 
2D histograms of the average $r_{\text {gas }}$ combined with a kernel estimator where there are few galaxies. The figure shows two main columns, on the left we show the results when limiting ourselves to a small range in physical aperture size, while the right hand column shows the same for a limited range in aperture size relative to half-light radius. In each row we have $2+2$ panels with the left panel in each pair showing the average $\log r_{\text {gas }}$ in each bin, while the right panel shows the mean redshift in each bin as a colour-scale and the completeness in each bin as a contour plot. We will discuss these further below but focus on the mean $r_{\text {gas }}$ maps first.

The top row shows the variation in $r_{\text {gas }}$ in the stellar mass versus stellar mass surface density plane. The top colour scale gives the median $\log r_{\text {gas }}$ at a given position and for ease of reading we have also overlain contour lines that are spaced 0.5 dex apart starting at $\log r_{\text {gas }}=-2$. What is particularly noticeable here is that the contour lines are not parallel to either coordinate axis which implies that neither $M_{*}$ nor $\Sigma_{*}$ alone are sufficient to predict the central gas content of these galaxies. This was also pointed out by Z09 and was one of their reasons for including a surface brightness term in their calibration for $r_{\text {gas }}$ versus colour, but note that their conclusion was relevant for the total gas content, here we show that a similar result is appropriate also for the central regions of galaxies. Also note that while the slope of the contour lines differ between the cut in physical size and in $r_{n}$, as one would expect from Fig. 18, the basic conclusion is still the same.

The second row shows the mean $r_{\text {gas }}$ in the plane of stellar mass versus rest-frame total $g-r$ colour. We see that galaxies with a particular $r_{\text {gas }}$ can have a range of colours but here there is a qualitative difference between a limit in physical size and in $r_{n}$. When we limit ourselves to the central $2 \pm 0.5 \mathrm{kpc}$, the gas content at fixed stellar mass does not vary much with integrated colour. In contrast, when we limit ourselves to a range in $r_{n}$, we find a clear correlation so that redder galaxies have less central gas at a fixed stellar mass.

The mass-metallicity plane is shown in the following row. This was examined also by Z09 and we find a similar diagram to those authors. What is particularly notable here is that there is a significant trend in $r_{\text {gas }}$ across the mean $M_{*}-Z$ relation (Tremonti et al. 2004). Also note the constant $r_{\text {gas }}$, particularly at $12+\log \mathrm{O} / \mathrm{H}>8.6$ and in the right column. This is consistent with the expectations of a closed-box model, but we note that this does not appear to hold everywhere in the $M_{*}-Z$ plane and indeed a full exploration of this result is outside the scope of the present paper.

The subsequent row shows the plane of stellar mass versus specific star formation rate, $\mathrm{sSFR}=\mathrm{SFR} / M_{*}$. We see clearly that the gas fractions of galaxies with the same $\mathrm{SFR} / M_{*}$ decline steadily with mass but that there is also a slight trend for galaxies at fixed $M_{*}$ to have declining gas fractions as the specific SFR declines, although the decline is not very strong. This is more visible when we focus on a range in $r_{n}$.

The penultimate row shows the gas fraction distributions as a function of position in the BPT diagram. The main point to note here is that the trend is smooth with position in the BPT diagram but with some trend across the main star forming ridge (c.f. Brinchmann et al. 2008) and decreases towards the composite/AGN branch.

The final row shows how the gas fraction change with position in the $\mathrm{D} 4000_{N}$ versus $\mathrm{H} \delta_{A}$ diagram. Recall that galaxies with high $\mathrm{H} \delta_{A}$ have undergone a significant burst in the last $\sim 1$ Gyr, while the average age of the stellar population typically increases with increasing D $4000_{N}$. The first point to note that along the median line the gas fractions decline steadily towards larger $\mathrm{D} 4000_{N}$. This median line is close to that expected for a slow declining star formation rate (e.g. Kauffmann et al. 2003b), while above it we expect to find post-burst systems.

While these $2 \mathrm{D}$ distributions really capture the interdependencies of various parameters, it is useful to simplify these into scaling relations. Focusing on the relationship with stellar mass and stellar surface mass density, we get

$$
\begin{aligned}
\log r_{\text {gas }}= & (8.76 \pm 0.03)-(0.474 \pm 0.004) \log M_{*} \\
& -(0.619 \pm 0.004) \log \Sigma_{*}
\end{aligned}
$$

with a $1 \sigma$ scatter of 0.27 dex. If one only includes the stellar mass in the fitting, the best-fit relation is

$\log r_{\text {gas }}=(8.17 \pm 0.03)-(0.925 \pm 0.003) \log M_{*}$

which has a $1 \sigma$ scatter of 0.33 dex. Qualitatively these relationships are similar to that found for total H I (e.g. Catinella et al. 2012) and CO (e.g. Saintonge et al. 2011a), but since the trends found here are for the central regions, they differ quantitatively, primarily in amplitude.

\subsection{The gas depletion time of galaxies in the nearby Universe}

Besides the fact that our method provides a measure of the gas content of the galaxies, one key advantage of our methodology over estimating gas content using the SchmidtKennicutt relation is that we can compare our gas content to the star formation rate. Since we have star formation estimates for exactly the same regions we have gas estimates for, the calculation of the gas depletion time,

$t_{R}=\frac{\Sigma_{\mathrm{gas}}}{\Sigma_{\mathrm{SFR}}} \approx \frac{M_{\mathrm{gas}}}{\mathrm{SFR}}$,

is straightforward in principle. In practice we rewrite equation (36) to give

$t_{R}=\frac{\text { Area }}{L_{\mathrm{H} \alpha}} \eta_{\mathrm{H} \alpha} \Sigma_{\text {gas }} \equiv \frac{\text { Area }}{L_{\mathrm{H} \alpha}} f_{t_{R}}$,

where Area is the area subtended by the 3" fibre in square parsecs and $\eta_{\mathrm{H} \alpha}$ is the conversion factor between $\mathrm{H} \alpha$ luminosity and the SFR (Charlot \& Longhetti 2001) defined through $\mathrm{SFR}=L_{\mathrm{H} \alpha} / \eta_{\mathrm{H} \alpha}$. We then calculate the likelihood distribution for $f_{t_{R}}$ and insert this in equation (37) to calculate a PDF for $t_{R}$. This accounts for intrinsic correlations between the parameters and we use this in the following. None of the conclusions below would change significantly if we were to use point estimates of the SFR and $\Sigma_{\text {gas }}$ and assume they were independent but the uncertainties would be more difficult to estimate and there is a slight overall bias in $t_{R}$ - this is expected, see appendix A in Brinchmann et al. (2004) for a discussion.

Fig. 20] is similar to Fig. 19] and shows how the median $t_{R}$ varies across the same parameter spaces. Comparing 

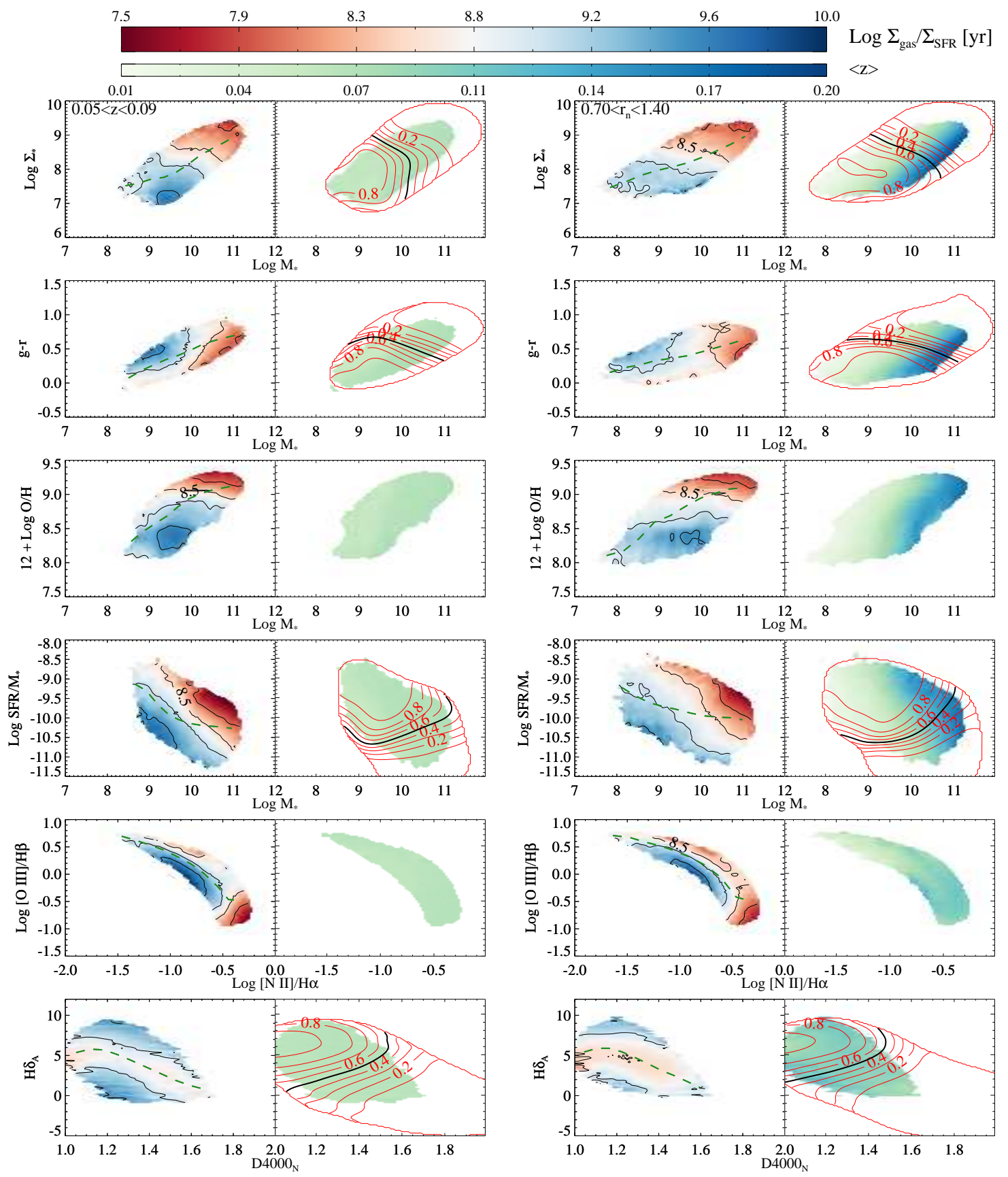

Figure 20. Similar to Fig. 19 but this time showing the median gas depletion time in each bin.

these two figures it is immediately obvious that long depletion times do not directly follow high gas fractions. Another immediate result is that galaxies that have very long depletion times are located in very particular parts of most diagrams. They have low $\Sigma_{*}$, are red for their mass, lie well below the median mass-metallicity relation but also have very low SFR $/ M_{*}$ for their mass as well as a low $[\mathrm{O}$ III $] 5007 / \mathrm{H} \beta$. We note in passing that a similar class of objects can be identified if one uses the Z09 calibration for gas mass estimation.
The $\mathrm{D} 4000_{N}-\mathrm{H} \delta_{A}$ diagram is particularly intriguing it shows that along the median trend, shown by the green dashed line, the galaxies have near constant depletion time of $\sim 10^{8}$ years in their central regions. However Fig. 19]shows that the gas fractions decrease steadily moving along the median curve from low to high $\mathrm{D} 4000_{N}$. This strongly hints at a regulatory mechanism for the central star formation rate, similar to that proposed to explain the same result for the total depletion times of galaxies by Schiminovich et al. (2010), see also the following section. 
The median (average) depletion time we are finding for the central $2 \mathrm{kpc}$ is 2.2 (3.6) Gyr and a similar result if were to limit ourselves in $r_{n}$ instead. We note that this is very close to the mean molecular depletion time, 2.35 Gyr, found on $\sim 1 \mathrm{kpc}$ scales by Bigiel et al. (2011) in nearby spiral galaxies with $\mathrm{CO}$ and $\mathrm{H}$ maps. Since the inner $2 \mathrm{kpc}$ is likely to have a very significant molecular component, these numbers should be comparable.

In contrast to Bigiel et al., however, we find a significant variation in depletion time with galaxy properties in the central regions. Instead our results match closely the strong variation in molecular depletion time with galaxy properties found by Saintonge et al. (2011b) in galaxies from the COLDGASS survey and we will return to this in a future paper.

The distribution of $t_{R}$ values in the $\log M_{*}-\mathrm{sSFR}$ diagram for the inner $2 \pm 0.5 \mathrm{kpc}$ is well fit by a simple linear function

$$
\begin{aligned}
\log t_{R}= & 8.99 \pm 0.37-(0.969 \pm 0.003) \log M_{*} \\
& -(0.948 \pm 0.004) \log \mathrm{sSFR}
\end{aligned}
$$

with a scatter of only $0.31 \mathrm{dex}$. In this equation, and in Figures 19 and 20 the stellar mass is the total stellar mass, while the specific star formation rate is calculated for the region sampled by the fibre, as is the depletion time. If the stellar mass is measured within the fibre, the coefficients would change to $(10.06,-1.04,-0.85)$ and if both the stellar mass and the specific SFR are the galaxy integrated values, the coefficients would be $(12.38,-1.10,0.73)$. It is important to emphasise that the gas content in these equations are for the central $2 \mathrm{kpc}$, and not for the galaxy as a whole; these are rather different quantities and we do not attempt to correct $t_{R}$ for aperture effects.

\subsection{Sample completeness and selection biases}

In the preceding section we have given an overview of the gas content and gas depletion times in the central regions of star-forming galaxies from the SDSS. However we are unable to measure the gas content of all galaxies and it is reasonable to ask to what extent our results are representative for the galaxy population and not significantly biased.

The main bias is that for a large number of galaxies we do not have sufficient emission lines detected in the spectra to apply our technique. There is also an additional biases for some galaxies with emission lines where the ionisation source is not likely to be stellar. In this case the modelling outlined in section 3 is not applicable. Thus there is a set of galaxies which likely have gas, but for which we cannot measure $\Sigma_{\text {gas }}$ using our method.

To illustrate the effect of the former bias, the second panel in each pair of panels in Figures 19 and 20 shows a contour map of the fraction of galaxies for which we could determine $\Sigma_{\text {gas }}$ with our method. This shows more or less the expected distribution: For blue and actively star forming galaxies we have $\Sigma_{\text {gas }}$ estimates for most galaxies, while for red, massive and low sSFR galaxies we only have $\Sigma_{\text {gas }}$ measurements for a very small subset.

A reasonable way to think about this is that where we have $\Sigma_{\text {gas }}$ for more than 50 per cent of the galaxies our mean $r_{\text {gas }}$ estimates likely to be fairly representative for the galaxy population as a whole. This level is indicated by a thicker black contour line in the plot. At lower completeness levels we do not know exactly how representative our measurements are, but as the main cause of incompleteness is a lack of star formation those missed galaxies most likely have lower gas content than those included in Figures 19 and 20. In this case $r_{\text {gas }}$ will be an upper limit to the true mean, but it is not immediately possible to say what the implications are for the depletion time.

A complementary method to test for the effect of incompleteness is to use a different technique to estimate the gas content and gas depletion time. We have two such methods available to us: gas content measurements from the ALFALFA survey and gas estimates from fitting formulae, in particular here the photometric method introduced by Z09. Unfortunately we can only use these methods to get total gas content so we cannot do a direct comparison here, but they are still very useful as a sanity check on the results.

We focus on two distributions, and in Fig. 21 we show the variation of $r_{\text {gas }}$ (top row) and $t_{R}$ (bottom row) in the $\log M_{*}$ versus $\log \Sigma_{*}$ plane for our method in the two leftmost panels in each row. These are the same displayed in Figures 19 and 20] and are therefore estimates of central gas properties. These can be contrasted to the total estimates using the Z09 estimator in the third panel of each row and ALFALFA in the final panel in each row.

The first point to note is that the $r_{\text {gas }}$ distributions are qualitatively similar in each panel. In particular we would expect that a $2 \mathrm{kpc}$ radius (leftmost panel) for the lowest mass galaxies would approach the total gas content and indeed we see a fairly close correspondence to the total estimates using the Z09 estimator. We also note that the ALFALFA sample is biased to more gas-rich galaxies overall so differs significantly from the rest.

The other striking result is that the distribution of $t_{R}$ is substantially different for the total estimator and the central. Again at low mass we can see a correspondence to the Z09 estimator and possibly also the ALFALFA measurement, but note that the colour scales are quite different.

A slightly different picture is offered by the $\mathrm{D} 4000_{N}$ versus $\mathrm{H} \delta_{A}$ plane shown in Fig. 22. The run of $r_{\text {gas }}$ is again qualitatively similar in each case. However in this case the $t_{R}$ distribution is also comparable and in particular we note that the trend for higher depletion times at both low and high $\mathrm{H} \delta_{A}$ at fixed $\mathrm{D} 4000_{N}$ that we noted above, can also be seen, albeit at a lower $\mathrm{S} / \mathrm{N}$.

This is a somewhat surprising result. The normal interpretation of strong Balmer absorption, here a high $\mathrm{H} \delta_{A}$, is that it indicates a recent cessation of or strong drop in star formation activity (e.g. Wild et al. 2009). What we are finding is that in these galaxies $r_{\text {gas }}$ is elevated (top row), particularly on a $2 \mathrm{kpc}$ scale and that this increase is not fully taken out by the increase in star formation, thus $t_{R}$ increases. What Fig. 22 implies is that many of these systems still retain significant amounts of gas but that as the star formation has dropped $t_{R}$ is high.

The population falling below the median line is less studied, but when matched in $\mathrm{D} 4000_{N}$ and stellar mass to other star forming galaxies they are seen to have a significantly, $\sim 0.7$ dex, lower star formation rate than galaxies on the median line. They are then concieveably rejuvenated post-starburst galaxies. 

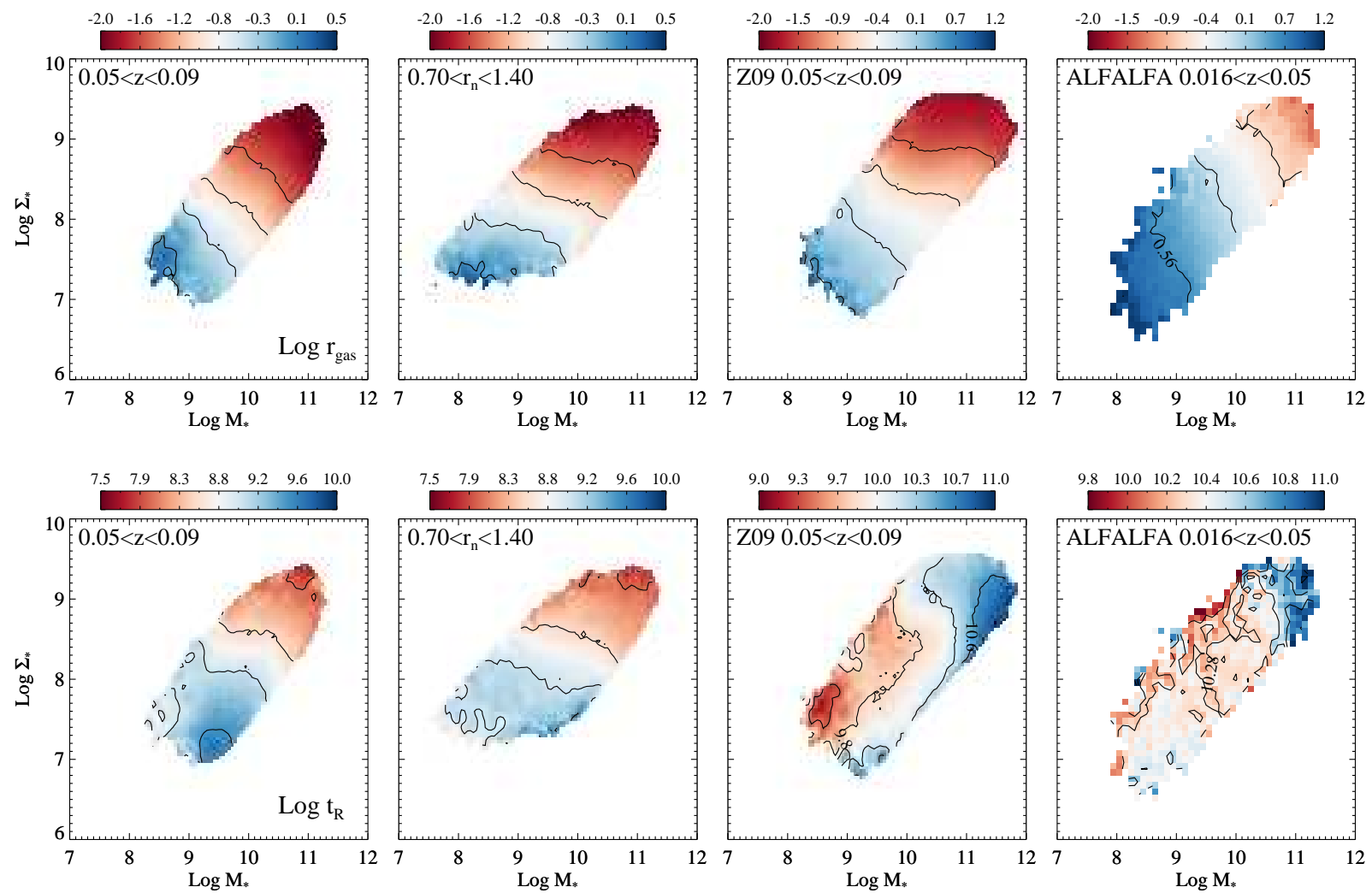

Figure 21. The variation of $r_{\text {gas }}$ (top row) and $t_{R}$ (bottom row) in the $\log M_{*}$ versus $\log \Sigma_{*}$ plane. The two left-most panels on each row are the same as shown in Figures 19 and 20 The third panel shows the mean $\log r_{\text {gas }}$ and $\log t_{R}$ in this plane using the Z09 photometric gas estimator, while the final panel shows the same using total gas masses from the ALFALFA $\alpha .40$ data release. Thus the two right-most panels show galaxy integrated quantities. Note that the behaviour for $t_{R}$ is clearly different for the total versus central measurements, while the $r_{\text {gas }}$ trends are qualitatively similar, but the quantitative levels are somewhat different as the colour bars above shows

These results are qualitatively consistent with a picture where most galaxies have a steady decline in gas content as they evolve and where inflow of gas leads to a star burst episode. In some cases when this ends, the star formation is quenched but all the gas is not removed from the system. The spread in $t_{R}$ on the mean $\mathrm{D} 4000_{N}-\mathrm{H} \delta_{A}$ track is quite substantial so the eventual fate of these systems cannot be well constrained from just this plot but this might offer an interesting constraint on the gas cycle in galaxies.

\section{CONCLUSIONS}

In the preceding we have presented a method for obtaining dust-to-metal and dust-to-gas ratios, as well as gas surface mass densities in galaxies using optical spectra. We showed that the dust-to-gas ratios provided by our method give consistent results to those obtained from modelling of the dust emission although a detailed one-to-one comparison was impossible with current data. We also showed that our method provides gas content estimates that agree with measurements of $\mathrm{H}_{2}+\mathrm{HI}$ to within a factor of $\approx 2$. This method requires emission lines that constrain the dust column density and lines of elements that are both (mostly) unaffected by depletion onto dust grains as well as elements that are depleted onto dust grains and that are important coolants in the interstellar medium. In practice this means that most strong optical emission lines from [O II] 3727 to [S II]6716,6731 should be measured.

The method provides total gas column densities unaffected by the substantial uncertainty on $X_{\mathrm{CO}}$, and because optical spectroscopy can provide much higher spatial resolution than most interferometric studies can, while at the same time providing star formation rates in the same region, this offers potentially a great complement to $\mathrm{CO}$ and $\mathrm{H}$ i studies of nearby galaxies. It would for instance be possible to compare our gas masses to $\mathrm{H} \mathrm{I}+\mathrm{H}_{2}$ masses to infer the CO-to- $\mathrm{H}_{2}$ conversion factor for large samples, similar to what was done for a sample of nearby galaxies by Lerov et al. (2011).

At the same time, rest-frame optical spectroscopy is fairly easy to carry out for star-forming galaxies over a wide range in redshift. As long as the spectra can be properly flux-calibrated, this opens up the possibility to trace the central gas content of galaxies over a wide range in redshift.

However we saw that there are a few limitations of the method: At metallicities below 50 per cent solar, our constraints on the dust-to-metal ratio are very weak and we need to apply a prior on this quantity. Overall this appears not to be a major issue as the effect of applying the prior is small and in comparison to the uncertainty in going from $\mathrm{CO}$ to $\mathrm{H}_{2}$ mass estimates, it is negligible.

The second issue is that at high gas column densities, 

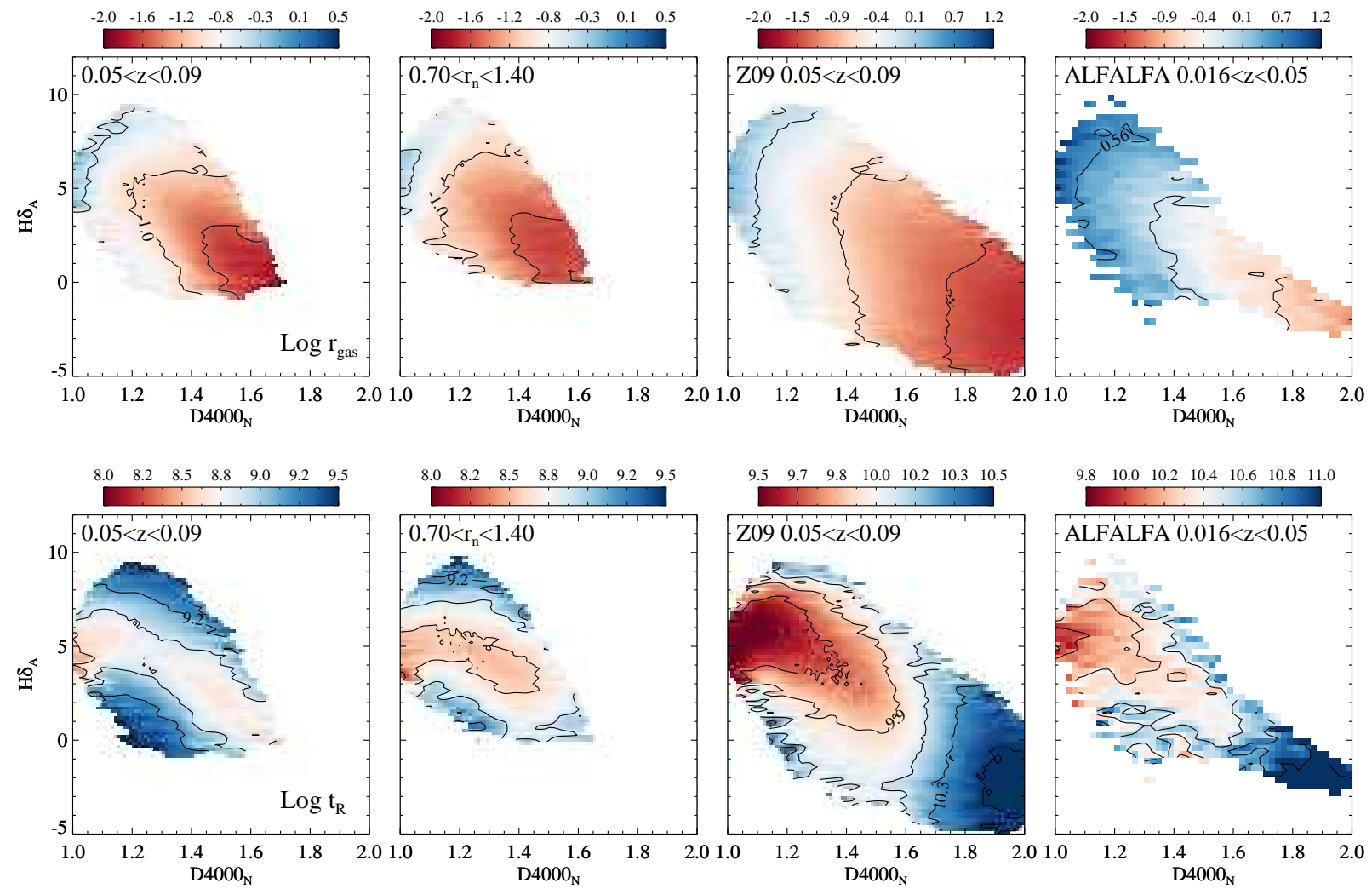

Figure 22. The same as Fig. 21 but for the $\mathrm{D} 4000_{N}$ versus $\mathrm{H} \delta_{A}$ plane. Note that there are clear similarities between the total and central distributions here, in particular a high depletion time both for high and low $\mathrm{H} \delta_{A}$ at fixed $\mathrm{D} 4000_{N}$.

$\Sigma_{\text {gas }} / \mathrm{M}_{\odot} \mathrm{pc}^{2}>75-100$, the method breaks down. This is simply because the emission lines must probe through the gas-rich region in order to provide information on it, but at very high column densities the regions become opaque to optical radiation. This is a fundamental limitation of this technique and does limit the usefulness of the technique when studying the very central regions of nearby galaxies. It is also likely that it will limit its usefulness to some classes of more distant galaxies although any firm conclusions on the latter must await a more careful assessment in future works. The method is also likely to break down at very low surface mass densities but we have not found this limit with our tests. From our tests we are confident that the method works well in the regime $10<\Sigma_{\text {gas }} / \mathrm{M}_{\odot} \mathrm{pc}^{-2}<50-70$, but the $\Sigma_{\text {gas }}$ estimates are well behaved at least down to $\Sigma_{\text {gas }} \sim 2 \mathrm{M}_{\odot} \mathrm{pc}^{-2}$ below which only 1 per cent of the SDSS DR7 galaxies fall, but we are unable to test this directly.

The final issue is that our method can only provide information on gas in regions where there is star formation going on. This is a key reason why this method cannot be a replacement for $\mathrm{CO}$ and $\mathrm{H}$ I measurements of galaxies. However this is offset by the fact that, particularly at higher redshifts, the method provides a much higher spatial resolution than $\mathrm{HI}$ and $\mathrm{CO}$ observations can provide, and it can account for atomic gas whose detection at significant redshifts is infeasible with current facilities, and thus complements the methods very nicely.

We demonstrated the usefulness of this method by applying it to the star-forming galaxies in the SDSS and pre- sented a summary of the variation in gas content and gas depletion times in the central $2 \pm 0.5 \mathrm{kpc}$ of these galaxies. These trends in general follow the trends for the overall gas content found in recent $\mathrm{HI}$ and $\mathrm{CO}$ surveys of nearby galaxies, so that the gas content of galaxies decreases with stellar mass and stellar mass surface density. However the large sample size has made it possible to highlight the complex variation of gas content and gas depletion time with physical parameters. In particular we found that galaxies with high and low Balmer absorption at a fixed D4000 $N$ appear to have high gas depletion times both in the central regions as well as in integrated quantities, possibly reflecting a feedback cycle associated with star bursting galaxies.

\section{ACKNOWLEDGEMENTS}

We thank Marijn Franx, Frank van den Bosch, Karl Glazebrook, Amelie Saintonge, Vivienne Wild, Richard Ellis, Kevin Bundy and Marc Verheijen, for discussions and input to this project. We thank J. Moustakas for providing emission line fluxes for SINGS galaxies in a machine readable form. We thank the anonymous referee for a constructive report that has helped improve the clarity of the paper.

This work has made use of SDSS data. Funding for the SDSS and SDSS-II has been provided by the Alfred P. Sloan Foundation, the Participating Institutions, the National Science Foundation, the U.S. Department of Energy, the National Aeronautics and Space Administration, the Japanese 
Monbukagakusho, the Max Planck Society, and the Higher Education Funding Council for England. The SDSS Web Site is http://www.sdss.org/.

The SDSS is managed by the Astrophysical Research Consortium for the Participating Institutions. The Participating Institutions are the American Museum of Natural History, Astrophysical Institute Potsdam, University of Basel, University of Cambridge, Case Western Reserve University, University of Chicago, Drexel University, Fermilab, the Institute for Advanced Study, the Japan Participation Group, Johns Hopkins University, the Joint Institute for Nuclear Astrophysics, the Kavli Institute for Particle Astrophysics and Cosmology, the Korean Scientist Group, the Chinese Academy of Sciences (LAMOST), Los Alamos National Laboratory, the Max-Planck-Institute for Astronomy (MPIA), the Max-Planck-Institute for Astrophysics (MPA), New Mexico State University, Ohio State University, University of Pittsburgh, University of Portsmouth, Princeton University, the United States Naval Observatory, and the University of Washington.

The colour maps in Figures 19, 22make use of ColorBrewer colour schemes from http://www. ColorBrewer.org (Brewer 2011).

We gratefully acknowledge the software utilised at various stages of this work: The Interactive Data Language (IDL), including the Astronomy Library maintained by Wayne Landsman at GSFC, the GAIA image analysis program and the Topcat table processing software (Tavlor 2005), both fruits of the now-defunct Starlink project. At times we have also made use of the Perl Data Language (http://pdl.perl.org), and much of the statistical analysis has used or built on the $\mathrm{R}$ programming language for statistical computing version 2.15.0 (http://www.R-project.org).

This research has made use of the NASA/IPAC Extragalactic Database (NED) which is operated by the Jet Propulsion Laboratory, California Institute of Technology, under contract with the National Aeronautics and Space Administration. This research has made use of the VizieR catalogue access tool, CDS, Strasbourg, France. The original description of the VizieR service was published in Ochsenbein et al. (2000).

\section{REFERENCES}

Abazajian K. N., et al., 2009, ApJS, 182, 543

Akritas M. G., Bershady M. A., 1996, ApJ, 470, 706

Andreon S., 2010, MNRAS, 1154

Aniano G. et al., 2012, ArXiv e-prints

Arimoto N., Sofue Y., Tsujimoto T., 1996, PASJ, 48, 275

Asplund M., Grevesse N., Sauval A. J., Scott P., 2009, ARA\&A, 47, 481

Baldry I. K., Glazebrook K., Driver S. P., 2008, MNRAS, 388, 945

Baldwin J. A., Phillips M. M., Terlevich R., 1981, PASP, 93, 5

Barnes D. G., et al., 2001, MNRAS, 322, 486

Bell E. F., de Jong R. S., 2001, ApJ, 550, 212

Bell E. F., McIntosh D. H., Katz N., Weinberg M. D., 2003, ApJL, 585, L117
Bigiel F., Leroy A., Walter F., Brinks E., de Blok W. J. G., Madore B., Thornley M. D., 2008, AJ, 136, 2846

Bigiel F. et al., 2011, ApJL, 730, L13

Blanton M. R., Lin H., Lupton R. H., Maley F. M., Young N., Zehavi I., Loveday J., 2003, AJ, 125, 2276

Blanton M. R., Roweis S., 2007, AJ, 133, 734

Bolatto A. D., Leroy A. K., Rosolowsky E., Walter F., Blitz L., 2008, ApJ, 686, 948

Boquien M. et al., 2013, ArXiv e-prints

Boselli A., Lequeux J., Gavazzi G., 2002, A\&A, 384, 33

Bothun G. D., 1984, ApJ, 277, 532

Boulanger F., Baud B., van Albada G. D., 1985, A\&A, 144, L9

Bresolin F., Gieren W., Kudritzki R.-P., Pietrzyński G., Urbaneja M. A., Carraro G., 2009, ApJ, 700, 309

Brewer C. A., 2011, http://www.ColorBrewer.org, accessed Nov 2011

Brinchmann J., Charlot S., White S. D. M., Tremonti C., Kauffmann G., Heckman T., Brinkmann J., 2004, MNRAS, 351, 1151

Brinchmann J., Ellis R. S., 2000, ApJL, 536, L77

Brinchmann J., Pettini M., Charlot S., 2008, MNRAS, 385, 769

Bruzual G., Charlot S., 1993, ApJ, 405, 538

Calura F., Dessauges-Zavadski M., Prochaska J. X., Matteucci F., 2009, ApJ, 693, 1236

Calura F., Pipino A., Matteucci F., 2008, A\&A, 479, 669

Catinella B., Haynes M. P., Giovanelli R., Gardner J. P., Connolly A. J., 2008, ApJL, 685, L13

Catinella B. et al., 2012, ArXiv e-prints

Catinella B., et al., 2010, MNRAS, 403, 683

Charlot S., Fall S. M., 2000, ApJ, 539, 718

Charlot S., Longhetti M., 2001, MNRAS, 323, 887

Cowie L. L., Songaila A., 1986, ARA\&A, 24, 499

da Cunha E., Charlot S., Elbaz D., 2008, MNRAS, 388, 1595

da Cunha E., Eminian C., Charlot S., Blaizot J., 2010, MNRAS, 403, 1894

Dame T. M., Hartmann D., Thaddeus P., 2001, ApJ, 547, 792

Dickinson M., Papovich C., Ferguson H. C., Budavari T., 2003, The Astrophysical Journal, 587, 25

Draine B. T., 2003, ApJ, 598, 1017

Draine B. T. et al., 2007, ApJ, 663, 866

Eales S. et al., 2012, ArXiv e-prints

Eales S. A., et al., 2010, A\&A, 518, L62+

Edmunds M. G., 2001, MNRAS, 328, 223

Erb D. K., Steidel C. C., Shapley A. E., Pettini M., Reddy

N. A., Adelberger K. L., 2006, ApJ, 646, 107

Ferland G. J., Korista K. T., Verner D. A., Ferguson J. W., Kingdon J. B., Verner E. M., 1998, PASP, 110, 761

Fukugita M., Ichikawa T., Gunn J. E., Doi M., Shimasaku K., Schneider D. P., 1996, AJ, 111

Galametz M., Madden S. C., Galliano F., Hony S., Bendo G. J., Sauvage M., 2011, A\&A, 532, A56

Genzel R. et al., 2012, ApJ, 746, 69

Gordon K. D., 2004, in Astronomical Society of the Pacific Conference Series, Vol. 309, Astrophysics of Dust, A. N. Witt, G. C. Clayton, \& B. T. Draine, ed., pp. 77-+ Gunn J. E. et al., 1998, AJ, 116, 3040

Gunn J. E., et al., 2006, AJ, 131, 2332

Haynes M. P. et al., 2011, AJ, 142, 170 
Helfer T. T., Thornley M. D., Regan M. W., Wong T., Sheth K., Vogel S. N., Blitz L., Bock D., 2003, ApJS, 145, 259

Hunter D. A., Walker C. E., Wilcots E. M., 2000, AJ, 119, 668

Inoue A. K., 2003, PASJ, 55, 901

Israel F. P., 1997a, A\&A, 317, 65

Israel F. P., 1997b, A\&A, 328, 471

Israel F. P., 2005, A\&A, 438, 855

Issa M. R., MacLaren I., Wolfendale A. W., 1990, A\&A, 236,237

Izotov Y. I., Stasińska G., Meynet G., Guseva N. G., Thuan T. X., 2006, A\&A, 448, 955

Jaffé Y. L., Poggianti B. M., Verheijen M. A. W., Deshev B. Z., van Gorkom J. H., 2012, ApJL, 756, L28

James A., Dunne L., Eales S., Edmunds M. G., 2002, MNRAS, 335, 753

Jenkins E. B., 1987, in Astrophysics and Space Science Library, Vol. 134, Interstellar Processes, D. J. Hollenbach \& H. A. Thronson Jr., ed., pp. 533-559

Jenkins E. B., 2009, ApJ, 700, 1299

Kannappan S. J., 2004, ApJL, 611, L89

Karachentsev I. D., Karachentseva V. E., Huchtmeier W. K., Makarov D. I., 2004, AJ, 127, 2031

Kauffmann G. et al., 2003a, MNRAS, 346, 1055

Kauffmann G., Li C., Heckman T. M., 2010, MNRAS, 409, 491

Kauffmann G., et al., 2003b, MNRAS, 341, 33

Kelly B. C., 2007, ApJ, 665, 1489

Kennicutt R. C., 1998, ApJ, 498, 541

Kennicutt R. C., Evans N. J., 2012, ARA\&A, 50, 531

Kennicutt, Jr. R. C. et al., 2003, PASP, 115, 928

Kennicutt, Jr. R. C., et al., 2007, ApJ, 671, 333

Kewley L. J., Ellison S. L., 2008, ApJ, 681, 1183

Kewley L. J., Groves B., Kauffmann G., Heckman T., 2006, MNRAS, 372, 961

Kroupa P., 2001, MNRAS, 322, 231

Kuno N. et al., 2007, PASJ, 59, 117

Leroy A. K. et al., 2009a, ApJ, 702, 352

Leroy A. K. et al., 2011, ApJ, 737, 12

Leroy A. K. et al., 2009b, AJ, 137, 4670

Leroy A. K., Walter F., Brinks E., Bigiel F., de Blok W. J. G., Madore B., Thornley M. D., 2008, AJ, 136, 2782

Lisenfeld U., Ferrara A., 1998, ApJ, 496, 145

Maraston C. et al., 2012, ArXiv e-prints

Marchesini D., van Dokkum P. G., Förster Schreiber N. M., Franx M., Labbé I., Wuyts S., 2009, ApJ, 701, 1765

McGaugh S. S., 1991, ApJ, 380, 140

Moustakas J., Kennicutt, Jr. R. C., Tremonti C. A., Dale D. A., Smith J.-D. T., Calzetti D., 2010, ApJS, 190, 233

Noordermeer E., van der Hulst J. M., 2007, MNRAS, 376, 1480

Noordermeer E., van der Hulst J. M., Sancisi R., Swaters R. A., van Albada T. S., 2005, A\&A, 442, 137

Noordermeer E., van der Hulst J. M., Sancisi R., Swaters R. S., van Albada T. S., 2007, MNRAS, 376, 1513

Ochsenbein F., Bauer P., Marcout J., 2000, A\&AS, 143, 23

Pagel B. E. J., Edmunds M. G., Smith G., 1980, MNRAS, 193, 219

Pforr J., Maraston C., Tonini C., 2012, MNRAS, 422, 3285
Puech M., Hammer F., Flores H., Delgado-Serrano R., Rodrigues M., Yang Y., 2010, A\&A, 510, A68+

Roberts M. S., 1969, AJ, 74, 859

Roberts M. S., Haynes M. P., 1994, ARA\&A, 32, 115

Roman-Duval J., et al., 2010, A\&A, 518, L74+

Saintonge A. et al., 2011a, MNRAS, 415, 32

Saintonge A. et al., 2011b, MNRAS, 415, 61

Salim S. et al., 2007, ApJS, 173, 267

Sánchez S. F., Rosales-Ortega F. F., Kennicutt R. C., Johnson B. D., Diaz A. I., Pasquali A., Hao C. N., 2011, MNRAS, 410, 313

Schiminovich D., et al., 2010, ArXiv e-prints

Schmidt M., 1959, ApJ, 129, 243

Seaton M. J., 1979, MNRAS, 187, 73P

Shapley A. E., 2011, ARA\&A, 49, 525

Shimasaku K. et al., 2001, AJ, 122, 1238

Smith J. A. et al., 2002, AJ, 123, 2121

Springob C. M., Haynes M. P., Giovanelli R., Kent B. R., 2005, ApJS, 160, 149

Taylor M. B., 2005, in Astronomical Society of the Pacific Conference Series, Vol. 347, Astronomical Data Analysis Software and Systems XIV, Shopbell P., Britton M., Ebert R., eds., p. 29

Tremonti C. A. et al., 2004, ApJ, 613, 898

Tully R. B., Verheijen M. A. W., Pierce M. J., Huang J.-S., Wainscoat R. J., 1996, AJ, 112, 2471

van der Hulst J. M., van Albada T. S., Sancisi R., 2001, in Astronomical Society of the Pacific Conference Series, Vol. 240, Gas and Galaxy Evolution, J. E. Hibbard, M. Rupen, \& J. H. van Gorkom, ed., pp. 451-+

Verheijen M. et al., 2010, ArXiv e-prints

Verheijen M. A. W., Sancisi R., 2001, A\&A, 370, 765

Walter F., Brinks E., de Blok W. J. G., Bigiel F., Kennicutt R. C., Thornley M. D., Leroy A., 2008, AJ, 136, 2563

Weingartner J. C., Draine B. T., 2001, ApJ, 548, 296

Wild V., Charlot S., Brinchmann J., Heckman T., Vince O., Pacifici C., Chevallard J., 2011, MNRAS, 417, 1760

Wild V., Kauffmann G., Heckman T., Charlot S., Lemson G., Brinchmann J., Reichard T., Pasquali A., 2007, MNRAS, 381, 543

Wild V., Walcher C. J., Johansson P. H., Tresse L., Charlot S., Pollo A., Le Fèvre O., de Ravel L., 2009, MNRAS, 395, 144

Yin S. Y., Liang Y. C., Hammer F., Brinchmann J., Zhang B., Deng L. C., Flores H., 2007, A\&A, 462, 535

York D. G., et al., 2000, AJ, 120, 1579

Young J. S., Scoville N. Z., 1991, ARA\&A, 29, 581

Young J. S. et al., 1995, ApJS, 98, 219

Zhang W., Li C., Kauffmann G., Xiao T., 2012, MNRAS, 425

Zhang W., Li C., Kauffmann G., Zou H., Catinella B., Shen

S., Guo Q., Chang R., 2009, MNRAS, 397, 1243

\section{APPENDIX A: APERTURE CORRECTIONS}

A key goal for our aperture correction scheme is to allow a direct comparison of spectroscopic gas mass measurements using the 3" SDSS fibre and H I mass estimates obtained with Arecibo, whose typical beam size is 3.5' full-width halfpower. Since the SDSS spectra rarely sample even 30 per cent of the total light of the galaxies and it is well-known 
that the H I disks often extend significantly outside the stellar disk, a direct comparison without taking into account aperture effects would be very misleading.

In order to compare the total H I gas masses to those derived from optical spectroscopy we have to account for differences in aperture. To carry out these aperture corrections we need $\mathrm{H}$ I and optical radial profiles for a wide range of galaxies. We take these from two samples observed using the Westerbork array: the Ursa Major sample (UMa) of Verheijen, Tully et al (Tully et al. 1996; Verheijen \& Sancisi 2001), and the WHISP survey (Noordermeer et al. 2005, 2007; Noordermeer \& van der Hulst 2007).

For the UMa survey we take surface photometry data from Tully et al. (1996) who presented results for $B, R$, $I$ and $K$. We match this data to the $\mathrm{HI}$ profiles from Verheijen \& Sancisi (2001), kindly supplied in electronic form by M. A. W. Verheijen. For the WHISP survey we take the H I profile fits reported by Noordermeer et al. (2005) and complement these with $B, R \& I$ profiles derived from surface photometry by Noordermeer \& van der Hulst (2007). For both surveys we ignore uncertainties in the fits as other uncertainties dominate.

Given the 2D distributions of $\mathrm{H}$ I and light we can calculate the gas-to-stellar mass ratio, $r_{\text {gas }}$ :

$r_{\text {gas }}(r<R)=\frac{\Sigma_{\text {gas }}(r<R)}{\mu_{*}(r<R)}$,

the gas fraction, $f_{\text {gas }}$ :

$f_{\text {gas }}(r<R)=\frac{\Sigma_{\text {gas }}}{\mu_{*}(r<R)+\Sigma_{\text {gas }}(r<R)}$,

and the surface gas mass density: $\Sigma_{\text {gas }}(r<R)$. Here we indicate explicitly that these values are integrated values inside a radius, $R$.

This allows us to calculate aperture effects for these quantities as a function of radius. Note that $f_{\text {gas }}$ and $r_{\text {gas }}$ are of course closely related since

$f_{\text {gas }}=\frac{r_{\text {gas }}}{1+r_{\text {gas }}}$

but we will treat them as two separate quantities as $f_{\text {gas }}$ depends on the absolute value of the stellar mass-to-light ratio, $M_{*} / L$, within the aperture, while $r_{\text {gas }}$ only depends on the relative value between the small and large aperture.

These quantities have quite different radial dependence, as illustrated in Fig. A1. As this figure shows (lower right panel), the behaviour of $\Sigma_{\text {gas }}$ might be far from monotonic which means that this quantity is not suited for aperture corrections. Fig. A1 also shows a series of dotted vertical lines. These show the radius sampled by the SDSS fibre at redshifts $0.02,0.05,0.1,0.2,0.5$ and 2.0 , respectively. Since the median redshift of the SDSS is close to 0.1, we can clearly see that aperture corrections will be substantial regardless of indicator chosen.

It turns out that both $r_{\text {gas }}$ and $f_{\text {gas }}$ are suitable for aperture correction, but as $r_{\text {gas }}$ does not depend on the absolute value of the $M_{*} / L$ ratio we choose to focus on this here and we write

$A_{r}=r_{\text {gas }}(R) / r_{\text {gas }}\left(R_{\text {tot }}\right)$,

where $R$ is the radius you wish to aperture correct to, and $r_{\text {gas }}\left(R_{\text {tot }}\right)$ is the radius corresponding to the total $r_{\text {gas }}$.

In the following we will derive a method to estimate $A_{r}$
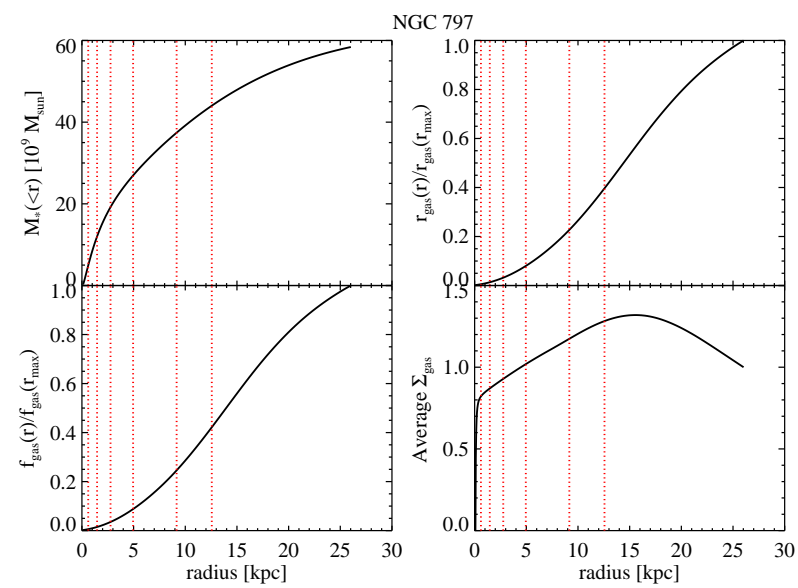

Figure A1. An illustration of the relevant profiles for aperture correction. The galaxy is NGC 797 from the WHISP sample and note that 'gas' here refers to H I only. The top left panel shows the enclosed stellar mass as a function of radius, using a fixed $M_{*} / L_{r}=1.78$. The dotted vertical lines which are the same in all panels, indicate the radius corresponding to the radius of the SDSS 3" fibres at $z=0.02,0.05,0.1,0.2,0.5$ and 2.0. The top right panel shows $A_{r}$ from equation (A4), the bottom left the corresponding $A_{f}$ for the gas fraction, and the bottom right the average gas column density inside radius $r, \Sigma_{\text {gas }}(\mathrm{r})$. From the figure it is clear that $\Sigma_{\text {gas }}$ does not show a monotonous trend so cannot be (easily) aperture corrected, while both $A_{r}$ and $A_{f}$ are relatively well-behaved.

for galaxies of different Hubble types, but it is worth commenting on $R_{\text {tot }}$ in equation (A4). This cannot be related to any quantity in the observed plane (such as the Arecibo) beam as that would give different aperture corrections for the same galaxy at different redshifts, so it has to be related to the physical properties of the galaxy. We will take it to be related to the radii containing 50 per cent and 90 per cent of the light below, but this is a significant source of systematic uncertainty which we will see below.

We will also ignore $M_{*} / L$ variations with radius, as well as the molecular contribution to the total gas mass here. This is a reasonable approximation in view of the systematic uncertainty just mentioned, but also because their effects are opposite, including molecular gas typically decreases $A_{r}$, while including $M_{*} / L$ variations typically increases $A_{r}$.

However $A_{r}$ shows a significant dependence on galaxy morphology. This is not unexpected as the gas content of galaxies has long been known to show a significant variation with Hubble type (e.g. Roberts \& Haynes 1994). This also extends to the distribution of gas in galaxies, and in Fig. A2 which shows all aperture correction profiles, $A_{r}$ for the combined WHISP and UMa sample.

The thick solid lines show for clarity the average profiles in four bins in T-type, which are from the top down, $[-1,1)$, $[0,2),[2,5)$ and $[6,10)$. As is clear from this figure there is a very noticeable type-dependence. However it is also a very clear spread around the mean. To explore the dependence on outer radius we also extrapolate the trends shown by fitting polynomials to the thick solid curves in Fig. A2.

To take the scatter properly into account we calculate the likelihood of a given aperture correction at a given radius for a given T-type, $P\left(A_{r} \mid R / R_{50}, T\right)$. The likelihood dis- 


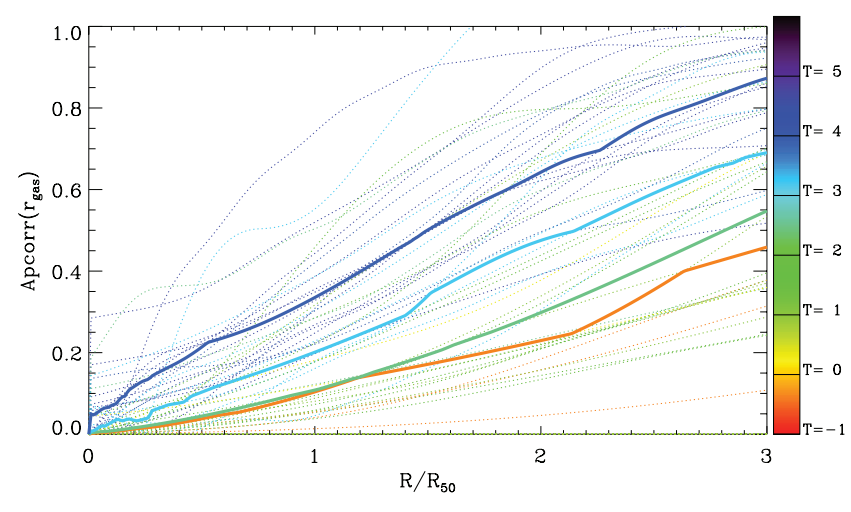

Figure A2. The aperture correction for $r_{\text {gas }}$ as a function of the radius and morphological type (indicated by colouring) for the combined WHISP and UMa samples. The thick solid lines show the average aperture correction profiles for four bins in T-type.

tribution is derived from the observed distribution of UMa and WHISP galaxies assuming an error of $0.1 \mathrm{in} A_{r}(R)$ and a type uncertainty of 1 class. When we apply this to the SDSS galaxies we convert the concentration, R90/R50, to Ttype using a linear fit to the data in Shimasaku et al. (2001) propagating the uncertainties on R90 and R50 to provide a likelihood distribution for $T$. We use this to marginalise $P\left(A_{r} \mid R / R_{50}, T\right)$ over $T$.

The result of applying this methodology to the galaxies in the SDSS with $z>0.01$ and H I detections from either S05, ALFALFA, or GASS, can be seen in Fig. A3 This figure compares the aperture corrected values on the $\mathrm{x}$-axis with our spectroscopic measures on the y-axis. To construct the main panel we have created a $2 \mathrm{D}$ PDF assuming the PDF of the quantity on the $\mathrm{x}$ and $\mathrm{y}$ axis are independent, a very reasonable assumption, and then added those 2D PDFs together. The shaded region shows the best linear fit to the data using the Bayesian fitting technique described in Appendix B with 68 per cent and 95 per cent posterior confidence intervals indicated. In the main panel we have assumed an outer radius for the $\mathrm{H}$ I equal to $4 \times R_{90}$, where $R_{90}$ is the radius containing 90 per cent of the $r$-band light.

The smaller panels show the effect of using other outer radii, with the top panel using the 1.75 ' radius of the Arecibo beam, and the three panels below using the indicated multiple of $R_{90}$. All aperture correction schemes using an outer radius equal to a multiple of $R_{90}$ results in a final relation that has a slope close to 1 (from 0.97 in the main panel to 1.06 for an outer radius equal to $R_{90}$ ), but with a systematic variation in the zero point. In contrast, assuming an outer radius equal to the Arecibo beam leads to a poor aperture correction and a best-fit line with slope 0.76 .

We conclude from this that while the unit slope is indicative of a good agreement between the aperture corrected H I content and our spectroscopic gas mass estimates, the systematic uncertainties in this comparison are sufficient to render this a rather weak test of our gas estimator.

\begin{tabular}{lcc}
\multicolumn{1}{c}{ Map } & Bin size & Kernel bandwidth \\
$\log M_{*}$ vs $\log \Sigma_{*}$ & {$[0.05,0.04]$} & {$[0.20,0.20]$} \\
$\log M_{*}$ vs $g-r$ & {$[0.05,0.02]$} & {$[0.20,0.10]$} \\
$\log M_{*}$ vs $12+\log \mathrm{O} / \mathrm{H}$ & {$[0.05,0.02]$} & {$[0.20,0.20]$} \\
$\log M_{*}$ vs $\log \mathrm{SFR} / M_{*}$ & {$[0.05,0.03]$} & {$[0.20,0.20]$} \\
$\log [\mathrm{N}$ II $] / \mathrm{H} \alpha$ vs $\log [\mathrm{O} \mathrm{III}] / \mathrm{H} \beta$ & {$[0.02,0.02]$} & {$[0.10,0.10]$} \\
$\mathrm{D} 4000_{\mathrm{N}}$ vs $\mathrm{H} \delta_{A}$ & {$[0.01,0.17]$} & {$[0.10,0.40]$}
\end{tabular}

Table C1. Bin sizes and kernel bandwidths used for the 2D gas maps in Figures 19- 22 Note that for ALFALFA the bin sizes is twice the width indicated here.

\section{APPENDIX B: BAYESIAN LINEAR REGRESSION WITH NON-GAUSSIAN ERROR BARS}

In Appendix $\mathrm{A}$ we need to fit a linear regression line for data with heteroscedastic and non-Gaussian error bars. In the case of heteroscedastic and Gaussian error bars, this is well known in the literature as fitting a linear functional relationship and has been used in astronomy for quite some time (e.g. Akritas \& Bershadv 1996; Kellv 2007; Andreon 2010).

Thus we write our equations as

$x=U+\epsilon_{x}$
$y=V+\epsilon_{y}=a+b U+$ noise

and where we assume that we know $\operatorname{PDF}\left(U+\epsilon_{x}\right)=\operatorname{PDF}_{x}(x)$ and $\operatorname{PDF}\left(V+\epsilon_{t}\right)=\operatorname{PDF}_{y}(a+b x)$. We can then write the probability distribution for the slope and intercept as:

$P(a, b \mid x, y) \propto P(x, y \mid a, b)=\operatorname{PDF}_{x}(x) \operatorname{PDF}_{y}(a+b x)$.

Where we have assumed uniform priors on $a$ and $b$, alternatively it might be better to use a uniform prior on the angle (e.g. Andreon 2010) but the constraints are tight on $b$ so it is not crucial here.

Given the small samples required we calculate the parameters on a grid rather than using Markov Chain Monte Carlo. We marginalise over $U$ and $V$ to get the PDFs for $a$ and $b$. To get the confidence interval in Fig. A3 we insert the PDFs for $a$ and $b$ in equation (B1).

\section{APPENDIX C: THE PRACTICALITIES OF CREATING 2D MAPS OF GAS CONTENT}

In section 6 we show a number of $2 \mathrm{D}$ maps of gas content and depletion time in nearby galaxies. For completeness and reproducibility this section describes in detail how these were constructed.

For each panel all objects that were classified as starforming using the BPT diagram and for which we have $\Sigma_{\text {gas }}$ estimates from the spectra and that satisfies the specific redshift or $R / R_{50}$ cuts are identified. For each diagram of $x$ versus $y$ we created a $2 \mathrm{D}$ histogram for these objects with 100 bins in each direction where we calculate the mean log $r_{\text {gas }}$ or $\log t_{R}$ in each bin. The resulting bin sizes are given in Table C1. We identify the bins with less than $N+\delta$ objects and for the objects that fall in these bins we use a $2 \mathrm{D}$ kernel density estimator using a Gaussian kernel with the bandwidths given in Table C1, again calculating the mean 


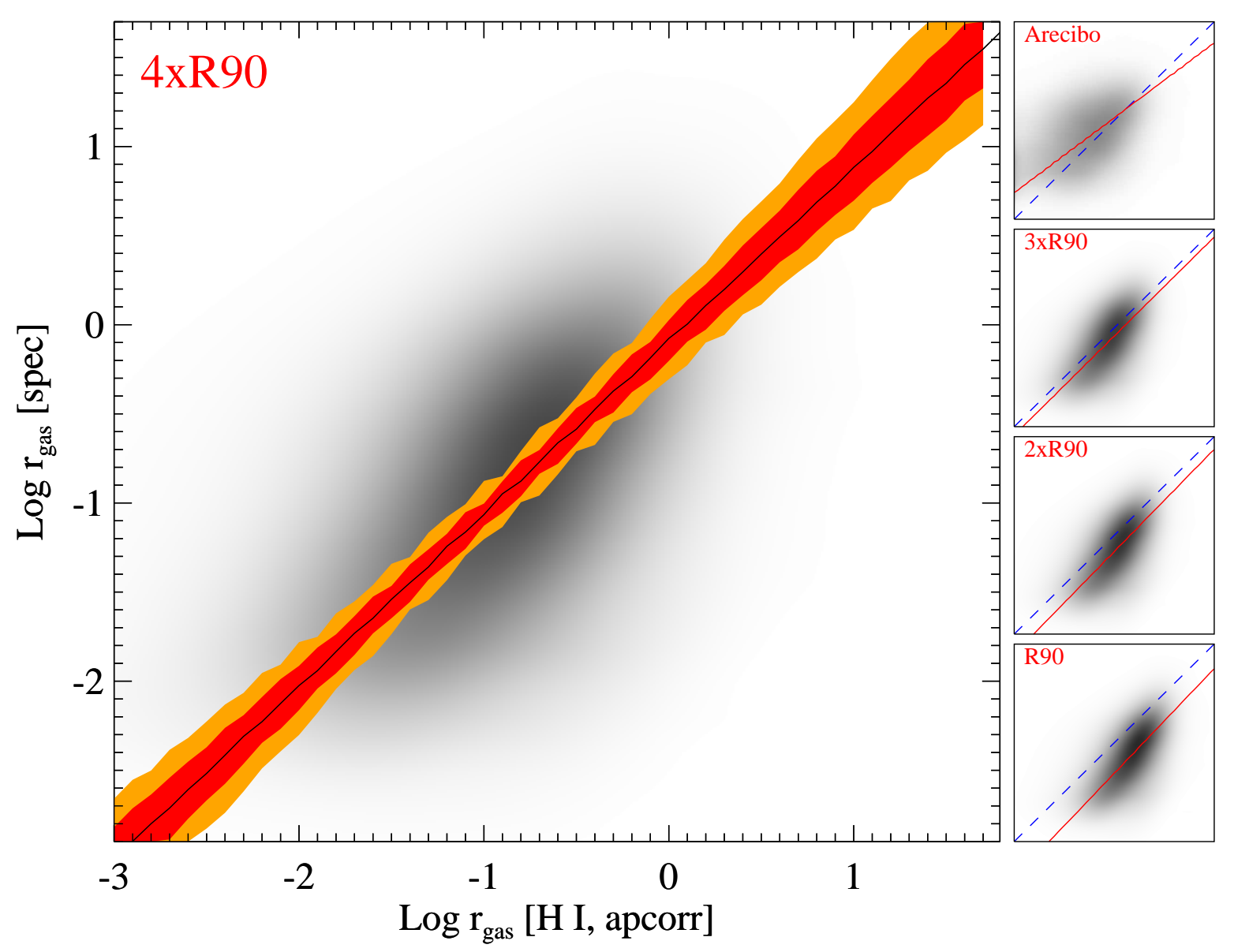

Figure A3. The main panel shows a comparison of $M_{\mathrm{H}} / M_{*}$, aperture corrected down to a 3" aperture, with our spectroscopic gas estimates. The grey scale shows the sum of the 2D PDF for this comparison, assuming independence. The shaded region shows the best fit linear fit to the data with the shading indicating 68 per cent and 95 per cent confidence intervals on the posterior predicted values (see Appendix $\mathrm{A}$ for details). For the main panel it was assumed that the total $\mathrm{H}$ I resides within 4 times the radius containing 90 per cent of the light, $R_{90}$, while the small panels on the side show the same for an outer radius of $R_{90}, 2 \times R_{90}, 3 \times R_{90}$ and that the outer radius is identical to the Arecibo beam. The solid red line in each panel is the median fit using the Bayesian method described in Appendix B while the dashed line shows the 1-1 line for comparison.

in each bin. We then replace all bins with less than $N$ objects with this kernel estimates. We use $N=100$ and $\delta=5$ for the plots in this paper. This combination of techniques gives good resolution where there are many objects while improving the $\mathrm{S} / \mathrm{N}$ in the regions with few galaxies. For display purposes we show the maps only where the sum of the kernel contributions to that bin is larger than 1 . We use the same method also for the maps using the Z09 photometric estimator and using the data from ALFALFA, but in the latter case we use 50 bins in each direction given the much smaller sample. 